\title{
GENERALIZATION OF WIDELY LINEAR FILTERING \\ CONCEPTS FOR EQUALIZATION AND \\ INTERFERENCE SUPPRESSION IN \\ PAM/QAM SYSTEMS
}

\author{
by \\ KIRAN KUMAR KUCHI
}

Presented to the Faculty of the Graduate School of

The University of Texas at Arlington in Partial Fulfillment

of the Requirements

for the Degree of

DOCTOR OF PHILOSOPHY

THE UNIVERSITY OF TEXAS AT ARLINGTON

May 2006 
Copyright (c) by Kiran Kumar Kuchi 2006

All Rights Reserved 
To my parents, my wife, and my son Nikhil 


\section{ACKNOWLEDGEMENTS}

This thesis work would not have been possible without the help and encouragement of many friends and colleagues. Foremost, I would like to thank my supervising professor Dr. Vasant Prabhu for motivating me to undertake doctoral studies while I was working at Nokia. I am fortunate to have him as a mentor and guide in both my professional and personal life. I also thank my committee members Dr. Bredow, Dr. Han, Dr. Lewis, and Dr. Manry for taking the time to serve in my dissertation committee.

I would also like to extend my appreciation to Nokia for supporting my doctoral studies. Specifically, I wish to thank Pekka Ranta, Riku Pirhonen, Eero Nikula, Olav Tirkkonen, Yrjo Kaipainen, Tony Reid, Sami Hoisko, and Seppo Yrjola for supporting my thesis work in various phases. I would like to thank my line managers at Nokia Craig Greer and Dennis McCain for letting me take time off towards my thesis work. I am indebted to my friend Gian Paolo Mattellini for introducing me to the basic concepts of widely linear filtering. The results of Chapter 3 and 4 can trace its roots to discussions with Gian Paolo and Aravind Ganesan. I benefited immensely from long discussions with my friend Charlie Zhang during the course of this thesis.

I would like to acknowledge two persons, my elementary school teacher Venkateswarlu Borra and my uncle Govindaiah Kuchi, who played a significant role in shaping up my character during the early part of my life. Finally, I am extremely grateful to my mother Vijaya and father Ramaiah Kuchi, my sisters Satyavani, Sravanthi and my beloved wife Srujana for their sacrifice, encouragement and patience.

April 20, 2006 


\author{
ABSTRACT \\ GENERALIZATION OF WIDELY LINEAR FILTERING \\ CONCEPTS FOR EQUALIZATION AND \\ INTERFERENCE SUPPRESSION IN \\ PAM/QAM SYSTEMS \\ Publication No. \\ Kiran Kumar Kuchi, Ph.D. \\ The University of Texas at Arlington, 2006
}

Supervising Professor: Vasant K. Prabhu

In PAM/QAM transmission systems, standard minimum mean square error (MMSE) linear (LE) or decision feedback equalization (DFE) methods are often used to suppress the detrimental effects inter-symbol and/or co-channel interference (ISI/CCI) effects of the channel. The main aim of this thesis is to show the benefits of widely linear (WL) filtering for equalization applications in wireless systems. For this purpose, we introduced generalized widely linear MMSE/MMSE-DFE equalization structures for both PAM systems that use real constellations and QAM (that use complex modulation alphabets) with multiple antennas and multiple co-channel interferers. In the proposed implementation, the WL receiver, unlike conventional methods, first separates the in-phase (I) and quadrature (Q) parts of the complex baseband received signal and jointly filters the two branches for signal detection. We derived the filter 
settings for both infinite and finite length implementation and analyzed the receiver performance in various channel conditions. This thesis has three main contributions. First, we analyze the advantage of WL filtering for PAM signaling in a white noise channel. When the ISI channel response is complex, we show that WL LE/DFE receivers outperform conventional methods both in complexity and performance. We obtained closed form mean square error (MSE) expressions that clarified the MSE advantage, and lower noise enhancement properties of WL receivers. Second, we show that WL receivers outperform conventional receivers in the presence of PAMtype interference. For both PAM and QAM signaling, assuming that the WL-DFE feedback path is error free, we show that, a receiver with $N$ antennas can reject up to $2 N-1$ PAM interferers or any combination of $M_{1}$ PAM and $M_{2}$ QAM interferers satisfying the constraint: $M_{1}+2 M_{2}<2 N$. This is significant gain compared to a conventional receiver whose interference cancellation (IC) capability is limited to $N-1$ interferers at most. Third, we analyze the symbol error rate (SER) performance and diversity-IC trade-off for the proposed receivers in a flat Rayleigh fading channel. After deriving an upper bound (UB) to the SER and certain approximations to the UB, we used the SER expressions to characterize the trade-off between diversity and interference cancellation. A comparison between conventional and WL methods showed that the additional dimensions created by I-Q split enhances the diversity gain in addition to providing IC advantage. 


\section{TABLE OF CONTENTS}

ACKNOWLEDGEMENTS ................... iv

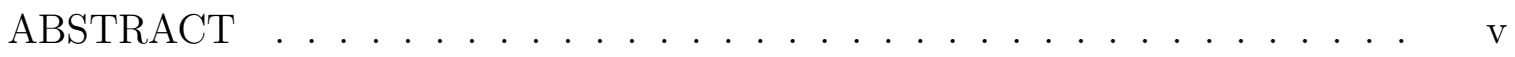

LIST OF FIGURES . . . . . . . . . . . . . . . . . . xi

LIST OF TABLES . . . . . . . . . . . . . . . . . . . xiii

Chapter

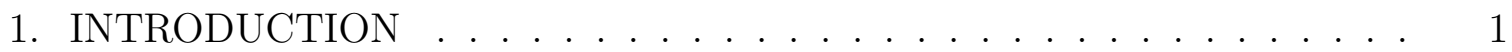

1.1 Background . . . . . . . . . . . . . . . . . 1

1.1.1 Characteristics of Wireless Channels . . . . . . . . . . . . 2

1.1.2 Conventional Equalization Techniques . . . . . . . . . . . . 3

1.1.3 Co-channel Interference Suppression . . . . . . . . . . . 5

1.1.4 Equalization using Widely Linear Filtering . . . . . . . . . 7

1.2 Overview of Thesis . . . . . . . . . . . . . . . . . . . . 12

1.3 Notation . . . . . . . . . . . . . . . . . . . . . 13

2. SYSTEM MODEL ........................... 15

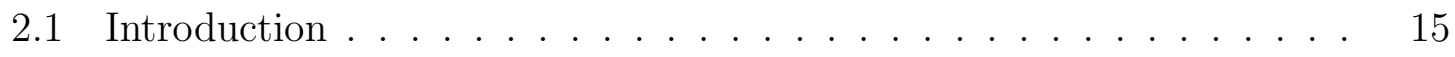

2.2 Signal Model . . . . . . . . . . . . . . . . . . . . . . 15

2.3 Signal Model with I-Q Split . . . . . . . . . . . . . . . 17

2.4 Interference Modeling . . . . . . . . . . . . . . . . . 20

3. WIDELY LINEAR EQUALIZATION FOR PAM SYSTEMS . . . . . . . . 24

3.1 Introduction . . . . . . . . . . . . . . . . . . 24

3.2 Linear Equalization . . . . . . . . . . . . . . . . . . . 25

3.2.1 Infinite Length Conventional Linear Equalizer . . . . . . . . . 25 
3.2.2 Infinite Length WL Linear Equalizer . . . . . . . . . . . . . . . 27

3.2.3 MSE Comparison for Infinite Length LE . . . . . . . . . . . . 28

3.2.4 Complexity Comparison . . . . . . . . . . . . . . . . . 32

3.2.5 Finite Length Conventional Linear Equalizer . . . . . . . . . . . 34

3.2.6 Finite Length WL Linear Equalizer . . . . . . . . . . . . . . . . 37

3.2.7 MSE Comparison for Finite Length LE . . . . . . . . . . . . . . 39

3.3 MMSE Decision Feedback Equalization . . . . . . . . . . . . . . . . . 41

3.3.1 Infinite Length Conventional DFE . . . . . . . . . . . . . . . . 41

3.3.2 Infinite Length WL DFE . . . . . . . . . . . . . . . . . 43

3.3.3 MSE Comparison for Infinite Length DFE . . . . . . . . . . . . . 45

3.3.4 Conventional Finite Length MMSE-DFE . . . . . . . . . . . . . 46

3.3.5 WL Finite Length MMSE-DFE . . . . . . . . . . . . . . . . . . 48

3.3.6 MSE Comparison for Finite Length DFE . . . . . . . . . . . . . 49

3.3.7 Complexity Comparison . . . . . . . . . . . . . . 51

3.4 Summary . . . . . . . . . . . . . . . . . . . 52

4. INTERFERENCE CANCELLATION . . . . . . . . . . . . . 53

4.1 Introduction . . . . . . . . . . . . . . . . . 53

4.2 WL MMSE-DFE for QAM Desired Signal . . . . . . . . . . . . 54

4.2.1 Symbol Detection _. . . . . . . . . . . . . . . 57

4.2 .2 WL LE for QAM . . . . . . . . . . . . . . . 58

4.3 Interference Cancellation Properties . . . . . . . . . . . . . . 59

4.3.1 Comparison between Conventional and WL DFE Receivers . . . . 60

4.3.2 Comparison between Conventional and WL LE Methods . . . . . 62

4.4 Complexity Aspects . . . . . . . . . . . . . . . . 64

4.4.1 Complexity of WL-PAM Receiver . . . . . . . . . . . . . . . 64

4.4.2 Complexity of WL-QAM Receiver . . . . . . . . . . . . 65 viii 
4.5 Simulation Results . . . . . . . . . . . . . . . . 66

4.5.1 Single Antenna Interference Cancellation _ . . . . . . . . . . 66

4.5.2 Two Antennas Results . . . . . . . . . . . . . . . . . . 69

4.6 Summary . . . . . . . . . . . . . . . . . . . . . 70

5. DIVERSITY ANALYSIS . . . . . . . . . . . . . . . 73

5.1 Introduction . . . . . . . . . . . . . . . . 73

5.2 Signal Model . . . . . . . . . . . . . . . . . . . . . 75

5.2.1 Interference Model . . . . . . . . . . . . . . . . 76

5.3 Receiver Structures . . . . . . . . . . . . . . . . . . . . . 77

5.3.1 Conventional Signal Combining _. . . . . . . . . . . 78

5.3.2 WL PAM Receiver Implementation in ISI free Channel . . . . . . 79

5.3.3 WL QAM Receiver Implementation in ISI free Channel . . . . . . 80

5.3.4 Structure of I-Q Noise plus Interference Correlation Matrix . . . . 81

5.4 SER for Conventional Receivers . . . . . . . . . . . . . . . . . . 82

5.4.1 Discussion ....................... 83

5.5 SER Analysis for WL Receivers . . . . . . . . . . . . . . . . . . . . 84

5.5.1 PEP for WL PAM Receiver . . . . . . . . . . . . . . . . 84

5.5.2 PEP for WL QAM Receiver . . . . . . . . . . . . . 85

5.5.3 Error Rate Analysis . . . . . . . . . . . . . . . . . . . 86

5.5.4 SER Approximations . . . . . . . . . . . . . . . . . . . . 88

5.6 Diversity-IC Trade-off . . . . . . . . . . . . . . . . . . . 91

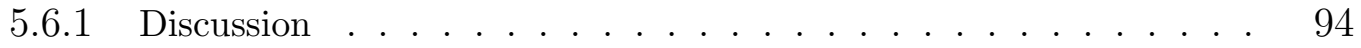

5.7 Simulation Results . . . . . . . . . . . . . . . . . . 95

5.8 Summary . . . . . . . . . . . . . . . . . . . . 97

6. FUTURE WORK . . . . . . . . . . . . . . . . . 100

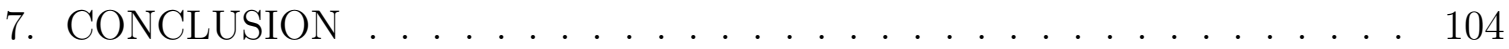


Appendix

A. MSE EXPRESSIONS FOR DFE . . . . . . . . . . . . . 107

B. SPECTRUM FACTORIZATION . . . . . . . . . . . . . 111

C. IC PROPERTIES . . . . . . . . . . . . . . . . . . . . 116

D. MSE EQUIVALENCE . . . . . . . . . . . . . . . . . . . 122

E. WISHART MATRICES . . . . . . . . . . . . . . . 126

REFERENCES . . . . . . . . . . . . . . . . . . . . . 129

BIOGRAPHICAL STATEMENT . . . . . . . . . . . . . . . . 136 


\section{LIST OF FIGURES}

Figure $\quad$ Page

1.1 A wireless link . . . . . . . . . . . . . . . . . 2

1.2 Widely linear filtering . . . . . . . . . . . . . . . . . . 8

1.3 Widely linear filtering using $\mathrm{I}-\mathrm{Q}$ processing $\ldots \ldots \ldots$

2.1 Conventional receiver . . . . . . . . . . . . . . . . . 16

2.2 Discrete baseband signal model . . . . . . . . . . . . . . . . . . 17

2.3 Signal model with $\mathrm{I}-\mathrm{Q}$ split $\ldots \ldots \ldots \ldots$

2.4 Equivalent baseband I-Q signal model for PAM . . . . . . . . . . . 18

2.5 Equivalent baseband I-Q signal model for QAM . . . . . . . . . . 19

3.1 Conventional linear equalizer . . . . . . . . . . . . . . . 25

3.2 WL linear equalizer for PAM . . . . . . . . . . . . . . . . 27

3.3 Conventional MMSE-DFE . . . . . . . . . . . . . . . . . . . 41

3.4 WL MMSE-DFE . . . . . . . . . . . . . . . . . . . . 45

4.1 WL MMSE-DFE for QAM . . . . . . . . . . . . . . 54

4.2 WL LE for QAM . . . . . . . . . . . . . . . . 59

4.3 Single antenna BPSK detection with single BPSK interferer . . . . . . 65

4.4 Single antenna BPSK detection with single 8-PSK interferer $\ldots \ldots .67$

4.5 Single antenna 8-PSK detection with single BPSK interferer $\ldots \ldots 68$

4.6 Dual antenna BPSK detection with 3-BPSK interferers . . . . . . . 68

4.7 Dual antenna BPSK receiver with mixed CCI (PAM+QAM) $\ldots . .69$

4.8 Dual antenna 8-PSK detection with single BPSK interferer . . . . . 70

4.9 Dual antenna 8-PSK detection with 3-BPSK interferers . . . . . . 71 
4.10 Dual antenna 8-PSK receiver with mixed CCI (PAM+QAM) . . . . . 72

5.1 Conventional receiver . . . . . . . . . . . . . . . . . . 77

5.2 WL receiver . . . . . . . . . . . . . . . . . . . . . . . 79

5.3 Single Antenna: BPSK desired-single BPSK CCI $\left(C=I_{0}=1\right) \ldots$

5.4 Single Antenna: BPSK desired-two BPSK interferers . . . . . . . . . 96

5.5 Single Antenna: QPSK desired-single BPSK CCI . . . . . . . . . . . . 97

5.6 Two Antennas: BPSK desired-2 interferers (PAM+QAM) . . . . . . 98

5.7 Two Antennas: BPSK desired-3 BPSK interferers $\left(C=I_{0}=1\right) \ldots 9$ 


\section{LIST OF TABLES}

Table $\quad$ Page

3.1 LE performance comparison in channel-1 . . . . . . . . . . . . . 40

3.2 LE performance comparison in channel-2 . . . . . . . . . . . . . 41

3.3 FIR DFE performance comparison in channel-1 . . . . . . . . . 51

3.4 FIR DFE performance comparison in channel-2 . . . . . . . . . . 51

4.1 IC comparison between conventional and WL receivers . . . . . . . 63

5.1 Diversity-IC trade-off for WL receivers with $N=2 \ldots \ldots 3$

5.2 Diversity-IC trade-off for WL receivers with $N=4 \ldots \ldots 3$

5.3 Diversity-IC trade-off for conventional receivers with $N=4 \ldots$. . . 93 


\section{CHAPTER 1}

\section{INTRODUCTION}

\subsection{Background}

In the past of few years, high demand for wireless services has led to a proliferation of wireless standards such as GSM, CDMA etc. In many of these systems, voice is a predominant service while data usage is expected to increase rapidly with the introduction of next generation wireless systems. It is estimated that nearly half the world population will soon have wireless phone access. Basic reliable voice communication has become all the more important factor in wireless system design especially to serve the billions of mobile phone users in emerging markets. In high (user) density cellular networks, the system has to be designed efficiently to provide high quality of service in a cost efficient manner. From radio design point of view, typical challenges faced by wireless networks include:

- Spectrum efficiency: Since bandwidth is scarce and expensive, the system has to be designed to maximize spectrum efficiency.

- Power efficiency: The network coverage typically depends on both power amplifier efficiency and the limited battery power of hand-held devices. Power efficient modulation schemes such as BPSK, MSK/GMSK (that have low peak to average ratio) are often used together with powerful channel codes to improve network coverage.

- Cost and size limitations: Cost and device size is a major issue in designing small portable units such as cellular phones. 
- Low implementation complexity: The complexity of baseband signal processing algorithms is an important consideration in DSP/ASIC implementation.

To address some of these problems, and to improve the quality and efficiency of wireless systems, many schemes such as MIMO (Multiple Input Multiple Output) [1], [2], SIMO (Single Input Multiple Output), many different Space-Time codes [3] and interference cancellation schemes have been proposed [4]. Almost all these schemes involve the use of multiple transmit/reciver antennas, complex receiver structures and complex signal processing schemes. In this thesis we propose a simple concept called generalized WL equalization that is applicable to both PAM-type signaling that uses real constellations and QAM-type signaling (complex constellations) that achieves almost the same enhancement as most of these other schemes at much less complexity.

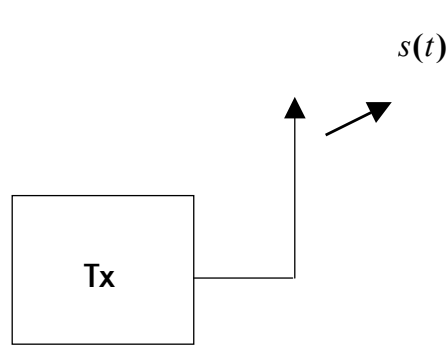

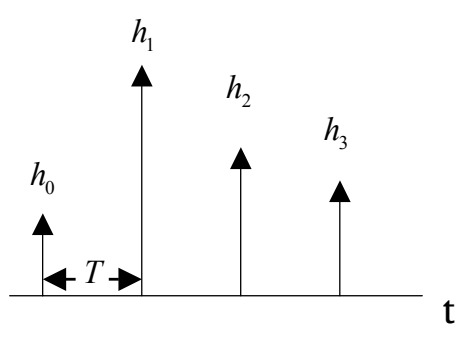

Propaqation channel $r(t)=s(t) \otimes h(t)+w(t)$

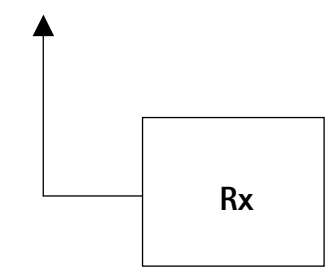

Figure 1.1. A wireless link.

\subsubsection{Characteristics of Wireless Channels}

A typical wireless link is shown in Figure 1.1. At the receiver, the information carrying signal $s(t)$ is often impaired by a multi-path fading channel, denoted as $h(t)$, and a co-channel interference term $w(t)$ that is generated by other users sharing the same channel (frequency re-use). As depicted in the figure, the fading channel is usually 
modeled as a Rayleigh fading process with (symbol spaced) statistically independent channel taps with unequal average power. The channel is said to be frequency selective when the symbol rate sampled channel response has dispersion; otherwise we call it a flat fading channel. The amount of dispersion in the channel (or the channel length) is mainly dictated by the transmission rate: $R=\frac{1}{T}$. For instance, the Typical Urban (TU) GSM channel (that has $270 \mathrm{KHz}$ bandwidth) can be modeled as a two tap channel whereas the Vehicular-A (1 MHz bandwidth) CDMA channel has up to six dominant channel taps. Although flat fading channel is ISI free, the signal undergoes severe fading. In such cases, the system has to employ some form of diversity such as transmit/receive diversity (using space-time codes) or time/frequency diversity (using signal repetition and channel coding). In frequency selective case, the channel inherently has some diversity. We can say that the diversity order of a frequency selective channel is equal to the total number of independently faded channel taps. A well designed equalizer would be able to fully capture this diversity thus reducing the need for other forms of diversity. In practice, the dispersive effects caused by the frequency selective channel/pulse shaping filters can very well be mitigated by single antenna receivers but it is generally difficult to suppress co-channel interference using a single antenna. Hence, multiple antenna receivers have been proposed as a remedy for the CCI problem. The generalized WL equalizers proposed in this thesis not only provide superior ISI suppression but also offer an interference cancellation gain in a single/multiple antenna system. Before discussing WL concepts, first we shall review the state of the art equalization techniques.

\subsubsection{Conventional Equalization Techniques}

For many modulation systems including PAM and QAM systems, conventional MMSE based equalization techniques [5], both linear and decision feedback equalization 
(DFE) methods, are used to solve the ISI problem. The linear equalizer is a preferred method for low complex systems where the noise enhancement caused by the LE is outperformed by the equalization gain. DFE, on the other hand, avoids the noise enhancement problem by using a combination of feed-forward and feedback filters. In general, DFE provides a signal-to-noise ratio (SNR) improvement over a LE. However, when there is significant energy in the DFE feed-back path, this method suffers from feedback errors. The maximum likelihood sequence estimator (MLSE) [6], [7] that estimates the whole data sequence by Euclidean distance minimization, is less sensitive to error propagation. But this algorithm has a computational complexity that grows exponentially with modulation size and channel length which makes it unsuitable for heavily dispersive channels. The maximum a-posteriori (MAP) equalizer [8] is similar to MLSE in performance but has an additional ability to provide symbol-by-symbol soft bit decisions which is a requirement in coded systems. Both MLSE and MAP have low complexity variants such as reduced state sequence estimators (RSSE) [9] which reduce the exponential complexity by using MMSE-DFE front-end pre-filtering and a combination of set partitioning and decision feedback techniques; RSSE is especially suitable for large modulation alphabet sizes and long ISI channels.

\subsubsection{Performance in Fading Channels}

Performance of any equalization scheme depends specifically on the type of ISI channel under consideration. Even with MLSE equalization, the ISI caused by the pulse shaping filters generally lead to a performance loss (except in some special cases) whereas the ISI caused by the frequency selective fading channel enhances performance through diversity effect. It can be shown that MLSE/MAP equalizers fully captures the diversity that is inherently present in the frequency selective channel. 
Analytical and simulation evaluation showed that MLSE provides full diversity (that is the diversity order of the given channel) whereas the conventional MMSE-DFE receiver (under the assumption of error free feed-back) provides nearly full diversity but with a slight SNR penalty (0.5-3.0 dB in typical channels of interest). Simulation studies also suggest that RSSE receivers with as few as 2-4 states [10] can recover most of the DFE loss and approach near MLSE performance in typical frequency selective channels. It is difficult to quantify the diversity order for a LE but simulation showed that this method also benefits significantly from multi-path diversity.

\subsubsection{Co-channel Interference Suppression}

In this thesis work we consider a TDMA type cellular system in which several users share a given channel on a time slot sharing basis (users do not interferer within a given cell). In this type of system CCI that arises from the frequency reuse mechanism will be a dominant impairment that limits the system capacity. In the present literature many different interference suppression schemes have been proposed to improve link and system performance. The IC gain is typically used to operate the network with lower frequency re-use factors (with high interference and high channel usage) to increase system capacity. In general, all CCI mitigation techniques requires some form of redundancy. This may be in the form of excess bandwidth, multiple antennas etc. As our main aim is to increase bandwidth efficiency, we consider a TDMA-type system without any excess bandwidth, but the receiver may exploit multiple antennas.

\subsubsection{Joint Detection Schemes}

The existing interference cancellation schemes may be broadly classified into two classes. The first approach, widely known as multi-user joint detection (JD), jointly detects both signal and interference using the channel state information (CSI) of both 
signals $[11],[12]$. As in single-user equalization, joint detection can be implemented using optimal MLSE/MAP techniques or sub-optimal low complexity MMSE/MMSEDFE techniques. In the presence of ISI and CCI, the MLSE type joint detector has additional complexity that grows exponentially with the total number of users (i.e. the total number of co-channel signals including signal and interference) and the channel memory [13]. On the other hand, MMSE/MMSE-DFE type joint detectors alleviate the exponential MLSE complexity using a front-end pre-filter to suppress the ISI first, followed by a joint DFE receiver that detects all users using multi-user decision feedback [14]. However, pre-filter based joint detectors work well only when the number of receiver antennas is greater than or equal to the total number of users whereas MLSE based JD can be useful even with a single receiver antenna. In flat Rayleigh fading channels, when CSI of all users is perfectly known at the receiver, it has been shown that many BPSK co-channel users can be separated using a single receiver antenna (approximately $2.0 \mathrm{~dB}$ SNR loss per user [15]). This result is unique to BPSK only; It was also shown in [15] that single antenna multi-user joint detection for QAM signals requires a very high SNR penalty unless multiple antennas are used. Note that the above mentioned multi-user equalization techniques explicitly rely on the CSI of both signal and interference for joint detection. In systems where the CSI of the interference cannot be obtained accurately (as in cellular systems), other means of interference cancellation have to be considered.

\subsubsection{Covariance based Interference Cancellation}

The second class of interference cancellation schemes, known as interference rejection combining (IRC) methods that are based on multi-antenna MMSE/MMSE-DFE type receiver processing, detect the signal of interest while treating the rest of the interference as background noise. These methods solely rely on interference covariance 
information for interference suppression. This approach has been used in cellular systems such as IS-54/136 (North American TDMA standard), GSM/EDGE [4], [16]. In [17], Winters et al. have shown that IRC receivers provide both diversity gain and interference suppression advantage when the receiver is equipped with multiple antennas. They have shown that, in flat fading channels, an $N$ antenna receiver can reject $M$ co-channel interferers $(M<N)$ while proving a diversity order: $N-M$. In frequency selective channels, simulation results indicate that, an MMSE-DFE receiver can also suppresses $M$ interferers but provides an increased diversity order roughly equal to: $\nu(N-M)$ where $\nu$ is the diversity order of the frequency selective channel under consideration. Note that IRC techniques are well suited for cellular type applications where it is difficult to obtain the CSI of interference accurately but the CSI of desired signal and the interference covariance can be accurately measured, for instance, using a training sequence.

The IRC method, however, has two limitations. First, these schemes cannot provide any IC advantage in single antenna systems. Second, in multiple antenna systems, the required number of antenna elements can be high if the system has a large number of dominant interferers. Note that in typical base station deployments, cost and complexity requirements generally limit the number of receiver antennas to 2-4, whereas a maximum of two receiver antennas has been considered at the terminal (for mobile handsets or lap-tops) side. In that respect, it is desirable to find techniques that offer a better complexity and performance trade-off.

\subsubsection{Equalization using Widely Linear Filtering}

Our work stems from a new signal processing concept called widely linear filtering that has successfully been used for linear (mean square) estimation of a signal and its complex conjugate. When the channel response is complex, collecting the complex 


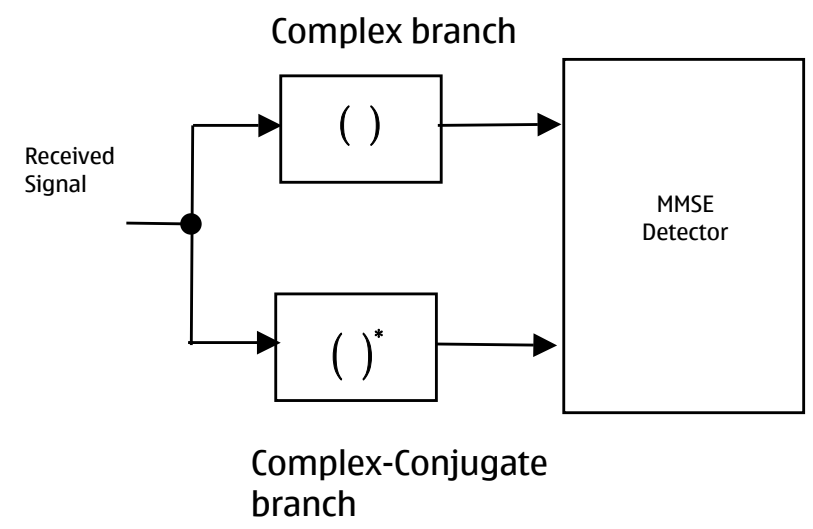

Figure 1.2. Widely linear filtering.

and complex-conjugate copies (alternatively collecting the real and imaginary parts) of the received signal creates two different copies of signal (see Figure 1.2). In [18], Picinbono and Chevalier showed that filtering of these two copies provides a performance advantage when the pseudo covariance (defined as covariance between the received signal and its complex conjugate) is non-zero. Such processes are classified as improper (or non-circular) random processes [19]. Although, complex, complexconjugate filtering was previously used by [20], [21] for signal processing and communication applications, the concept has not received much attention until Picinbono formally introduced the notion of widely linear filtering (WLF). Though, complex, complex-conjugate based implementation has been a popular choice for WLF, the same benefit can be obtained using an alternative implementation based on the real (I) and quadrature $(\mathrm{Q})$ parts of the received signal as shown in Figure 1.3. The I-Q implementation is computationally more efficient as the filter tap calculations and signal filtering involves real arithmetic only.

Let us now examine the suitability of commonly used PAM and QAM signals for WLF. Conventional PAM systems use one dimensional (real) modulation alphabets that are modulated on either the cosine or sine branches were as QAM uses two di- 


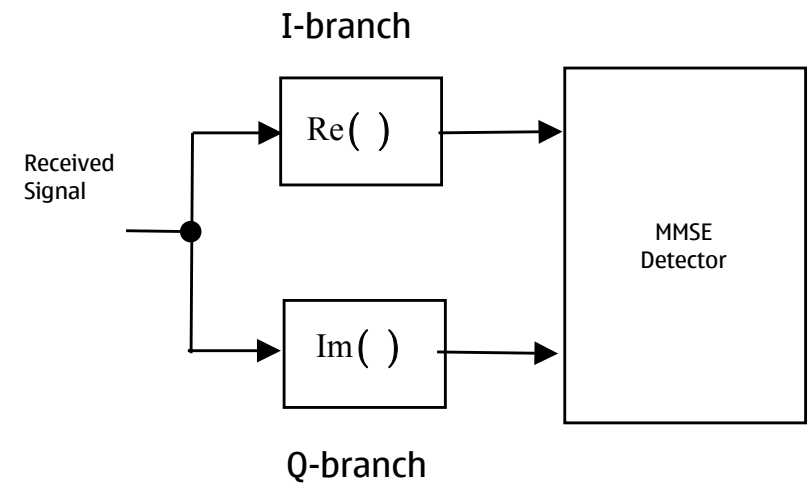

Figure 1.3. Widely linear filtering using I-Q processing.

mensional (complex) symbols whose real part is modulated on to the in-phase (sine) and the imaginary part is sent on the quadrature (cosine) branch. In many systems, including the wireless case, the received sine and cosine signals undergo different attenuations during radio propagation. Therefore, the baseband channel impulse response can be modeled as a complex response with distinct real and imaginary parts. Also, other modulation schemes such as MSK/linearized GMSK [22] use both inphase and quadrature branches for modulation but the signal carries real information symbols. These schemes have an equivalent PAM representation (after applying a 90 degree phase de-rotation between successive symbols). Especially in case of GMSK, because of ISI introduced by the modulator, the de-rotated channel response takes complex values. In general, when the channel impulse response is complex (either due to propagation channel or pulse shaping), we can show that, PAM becomes a non-circular random process as it exhibits non-zero pseudo-covariance whereas QAM becomes circular (the pseudo-covariance function vanishes for symmetric QAM constellations). Essentially, WLF can be considered in systems where either wanted signal or interference involves PAM-type signals. 


\subsubsection{Related Work}

Yoon and Leib, in [23], showed the advantage of WLF using an SNR maximizing matched filter receiver that takes into account the pseudo covariance of the noise (plus interference) in a two user DS-CDMA system employing real valued spreading sequences and BPSK modulation. Subsequently, Lampe and Breiling, in [24], generalized these results to a multi-user case. They showed that, in a DS-CDMA system that uses complex spreading sequences and WL multi-user detectors, PAM signaling offers a higher spectral efficiency than QAM. In addition, Lampe et al. in [25] proposed an interesting application of WLF for iterative multi-user detection for QAM systems. Though, a symmetric QAM constellation is a circular random process by it self, in the presence of extrinsic information (i.e. when a-priori information is available as in an iterative receiver), the QAM signal becomes non-circular. This property was used to improve the convergence speed of (i.e. to reduce the number of iterations) an iterative multi-user receiver. Also, in [26], WLF was applied to suppress narrow band interference (NBI) in DS-CDMA systems that uses either PAM or QAM signals. Further, [27] extended that concept to NBI cancellation in multi-carrier systems.

Recently, in [28], Gerstacker et al. showed that, when the desired signal has PAM and when the ISI channel response is complex (noise being AWGN), widely linear equalizers (WLE), both WL-LE and WL-DFE, provide a performance advantage over conventional equalizers. It was shown that WL receivers that independently filter the complex and complex-conjugate parts of the received signal experience less number of channel zeros (near unit circle). This property was shown to be the reason for the lower noise enhancement and better performance of WL receivers. In [29], this concept was extended to equalization of space-time block codes with QAM type signaling. In this case, the gain of WL receivers owes to their ability to exploit 
the inherent correlations between the complex and complex-conjugated copies of the QAM symbols present in the Alamouti space-time block code. Although, the WLE concepts proposed in [28], [29], in principle, can be extended for CCI suppression, the authors did not address this aspect; they specifically considered performance in a white noise channel with ISI.

With regard to CCI suppression, GSM is one of the first industrial standards to adopt WLF [30]. In [31], Trigui and Slock applied I-Q type WL receivers for CCI cancellation for single antenna GMSK receivers, whereas in [32], Gardner and Reid applied a modified version of WLF (so called linear-conjugate-linear (LTL) FRESH filters) for CCI cancellation in GSM with single/multiple antennas ${ }^{1}$. However, most of the existing WLE methods address IC concepts for systems that use BPSK modulation or PAM-type modulation only. An important case where QAM being the desired signal and PAM being interference has not been considered. A practical application of this type of channel occurs in GSM/EDGE type systems that uses a combination of PAM (GMSK) and QAM (8-PSK) signals. In this type of system, scenarios arise, where the desired signal may have PAM/QAM, but interference consists of a mixture of PAM and QAM signals. In this channel, conventional WLE that are tailored for PAM signaling are not useful. Moreover, extension of WL concepts to QAM requires a new equalization framework. This thesis addresses this problem and further extends the WL equalization method to multiple antennas and multiple interferers. We show that WL processing is not only useful for PAM systems but also provides significant advantage for QAM systems especially in the presence of PAM-type interference.

\footnotetext{
${ }^{1}$ Note that single antenna interference cancellation (SAIC) is a mandatory feature for GMSK modes of GSM standard.
} 


\subsection{Overview of Thesis}

In this thesis, we consider receiver design for a single/multiple antenna receiver but the transmitter is assumed to have a single antenna only. We depart from the conventional complex, complex-conjugate type WL approach and introduce alternative and more efficient WL-DFE/WL-LE receiver structures for PAM/QAM systems using the inphase (I) and quadrature (Q) parts of the received signal collected from each antenna branch. We derive the receiver settings for both finite and infinite length filters and analyze the performance under various channel conditions. The thesis is organized into six chapters.

After introducing the over-sampled baseband signal model in Chapter 2, in Chapter 3 we first analyze the performance of WL PAM equalizers in ISI channels with white noise. In this chapter we provide detailed derivations of WL MMSE/MMSE-DFE filter settings for finite and infinite length cases. We demonstrated how WL receivers take advantage of additional dimensions created by I-Q split to reduce the noise enhancement problem that is inherent to MMSE receivers. The performance advantage is clarified by comparing the MSE for conventional and WL methods. In addition, the computational advantage of WL methods is highlighted.

In Chapter 4 we consider performance in interference limited systems. Specifically, we consider an interference limited channel where CCI consists of a mixture of PAM and QAM interferers. This is the baseline model for rest of the interference analysis throughout this thesis. In this chapter, we first formulated WL MMSE/MMSE-DFE receivers for QAM/PAM systems with multiple antennas and multiple (PAM/QAM) interferers. Next, we derived the IC properties of WL receivers as function of number of antennas and the number of interferers. We used simulation to show the BER 
advantage of proposed receivers for single and dual antenna systems in frequency selective Rayleigh fading channels.

In Chapters 3, 4 we reviewed the WL equalization problem mainly from signal processing point of view. For channels with fading and interference, it is equally important to understand the error rate behavior and the diversity-IC trade-off [17]. This aspect is well understood for conventional systems. However, the analogous results for WL receivers are not available in the literature. In Chapter 5 we first derive an upper bound to the symbol error rate of a PAM/QAM WL receiver in flat Rayleigh fading channels. Using the SER analysis, we established the diversity-IC trade-off for WL receivers with multiple antennas and multiple (PAM/QAM) interferers. Specifically, we show that, when interference consists of $M_{1}$ PAM and $M_{2}$ QAM interferers, an $N$ antenna PAM/QAM WL receiver provides full IC with a diversity order: $N-M_{2}-\frac{M_{1}}{2}$. This is significant gain compared to conventional methods that provide full IC gain up to $M$ interferers with a diversity order: $N-M$ for $M<N$ where $M=M_{1}+M_{2}$. Finally we outlined a list of future research topics in Chapter 6 followed by conclusions in Chapter 7.

\subsection{Notation}

The following notation is adopted throughout this thesis.

- Matrices $(\mathbf{H})$ are denoted with upper case boldface letters.

- Vectors $(\mathbf{h})$ are denoted with lower case bold face letters.

- Scalar quantities $\left(R_{x x}(f)\right)$ are denoted with non boldface letters.

- The notation $\operatorname{Tr}(\mathbf{H}), \mathbf{H}^{*}, \mathbf{H}^{\prime}, \mathbf{H}^{\dagger}$, and $|\mathbf{H}|$ denotes trace, conjugate, transpose, conjugate-transpose, and determinant operations respectively. 
- The symbol $\circledast$ denotes element wise convolution between any two matrices or vectors.

- $h(D)=\sum_{k} h_{k} D^{k}$ denotes the discrete D-transform whereas the sequence $h_{-k}^{*}$ has a D-transform $h^{*}\left(D^{-*}\right)=\sum_{k} h_{k}^{*} D^{-k}$. By letting $D=e^{-j 2 \pi f T}, \quad \frac{-1}{2 T} \leq f \leq$ $\frac{1}{2 T}$ we get the corresponding discrete Fourier transform (DFT).

- We use the notation $h(f), h^{*}(-f)$ that correspond to $h(D), h^{*}\left(D^{-*}\right)$ respectively.

- The notation $h_{k} \leftrightarrow h(f)$ denotes a DFT pair.

- The notation $\operatorname{Re}\left(\mathbf{y}_{k}\right), \operatorname{Im}\left(\mathbf{y}_{k}\right)$ denotes the real and imaginary parts of a complex valued vector $\mathbf{y}_{k}$ respectively.

- Vectors/matrices with a super script $\tilde{\mathbf{h}}_{k}$ or $\tilde{\mathbf{H}}_{k}$ is used to denote I/Q domain quantities only.

- The convolution between two functions $x(t)$ and $y(t)$ is defined as: $x(t) \circledast y(t) \triangleq$ $\int_{\tau} x(\tau) y(t-\tau) d \tau$.

- The convolution between two discrete functions $x_{k}$ and $y_{k}$ is defined as: $x_{k} \circledast y_{k} \triangleq$ $\sum_{m} x_{m} y_{k-m}$

- $\operatorname{diag}\left(x_{1}, x_{2}, \cdots, x_{N}\right)$ denotes an $N \times N$ diagonal matrix with entries $x_{0}, . ., x_{N}$ along the main diagonal and zeros elsewhere.

- Let $\mathbf{x}=\left[x_{1}, x_{2}, . ., x_{N}\right]^{\prime}$ denote a real Gaussian random (column) vector of length $N \times 1$. Let $E[\mathbf{x}]=\mathbf{m}_{x}, E\left[\left(\mathbf{x}-\mathbf{m}_{x}\right)\left(\mathbf{x}-\mathbf{m}_{\mathbf{x}}^{\dagger}\right)\right] \triangleq \mathbf{R}_{x x}$ denote the mean and covariance functions respectively. The joint probability density function (pdf) of elements of $\mathbf{x}$ is given by: $p\left(x_{1}, x_{2}, . ., x_{N}\right)=\frac{1}{\sqrt{(2 \pi)^{N}\left|\mathbf{R}_{x x}\right|}} e^{-\left(\mathbf{x}-\mathbf{m}_{x}\right)^{\dagger} \mathbf{R}_{x x}^{-1}\left(\mathbf{x}-\mathbf{m}_{x}\right)}$. This density function is compactly denoted as: $N\left(\mathbf{m}_{x}, \mathbf{R}_{x x}\right)$.

- The symbol $E[$.$] denotes the expectation operation with respect to all the ran-$ dom variables contained in that function whereas $E_{x}[\cdot]$ denotes expectation operation explicitly with respect to random variable $x$. 


\section{CHAPTER 2}

\section{SYSTEM MODEL}

\subsection{Introduction}

In our system model we consider a single antenna transmitter and a receiver with $N$ antennas. In this chapter we first introduce the signal model for conventional oversampled systems in Section 2.2. In Section 2.3 we introduce the discrete baseband I-Q signal model for PAM and QAM systems. As we are interested in determining receiver performance in both interference and noise limited situations, in Section 2.4 we model the additive noise as a sum of several PAM and QAM interferers and thermal noise. Using the signal model developed in this chapter, we first consider conventional and WL receiver structures for PAM signaling in a white noise channel in Chapter 3. In Chapter 4, the WL receiver concepts will be extended to QAM systems with multiple antennas and multiple CCI.

\subsection{Signal Model}

We consider an M-ary PAM/QAM-type system when the symbol transmission rate $R=\frac{1}{T}$, the modulated/transmitted signal $s(t)$ is,

$$
s(t)=\sum_{k=-\infty}^{k=\infty} x_{k} p(t-k T) .
$$

The symbols $x_{k}$ takes real values for PAM, and complex values $x_{k}=x_{k, I}+j x_{k, Q}$ in case of QAM, and $p(t)$ is assumed to be a real-valued pulse. The receiver has $N$ antennas. The propagation channel between the transmitter and $i$ th receiver antenna 


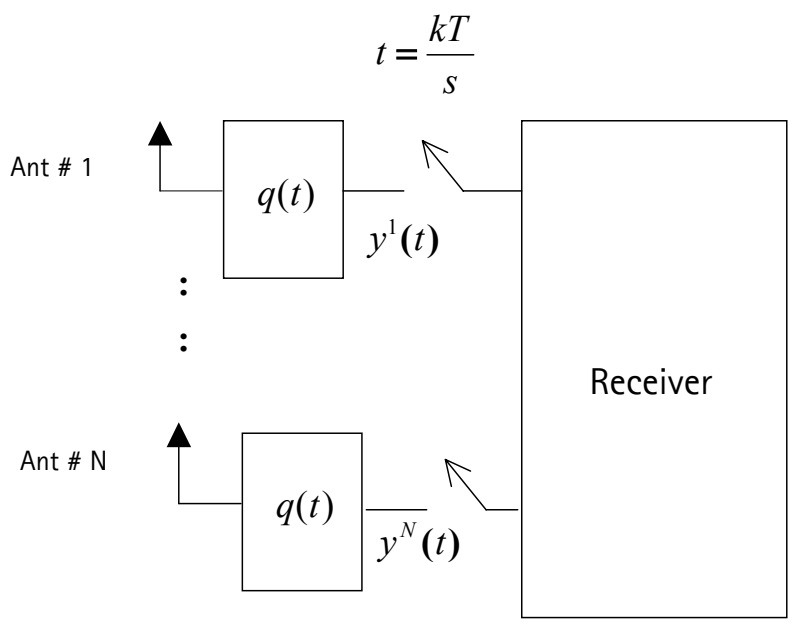

Figure 2.1. Conventional receiver.

has a complex-valued response $f^{i}(t)$, the signal is corrupted by additive noise term $z^{i}(t)$. Then the output of a front-end (anti-aliasing) filter $q(t)$ denoted as $y^{i}(t)$ at the $i$ th antenna can be written as (see Figure 2.1):

$$
y^{i}(t)=\sum_{k=-\infty}^{k=\infty} x_{k} h^{i}(t-k T)+n^{i}(t), \quad i=1,2, . ., N
$$

where

$$
h^{i}(t) \triangleq p(t) \circledast f^{i}(t) \circledast q(t)
$$

denotes the total channel response which is the convolution of transmitted pulse, propagation channel, and the receiver filter and

$$
n^{i}(t)=z^{i}(t) \circledast q(t)
$$

denotes the filtered noise term, $z^{i}(t)$ being the additive noise component that is collected at the $i$ the antenna. Assuming an over-sampling rate of $\frac{s}{T}$, we sample the received waveform at $t=k T+l \frac{T}{s}, l=0,1, \ldots,(s-1)$ to obtain

$$
\begin{aligned}
y_{k, l}^{i} & =y^{i}(t)_{\mid t=k T+l \frac{T}{s}} \\
& =\sum_{m=0}^{v} x_{k-m} h_{m, l}^{i}+n_{k, l}^{i}
\end{aligned}
$$




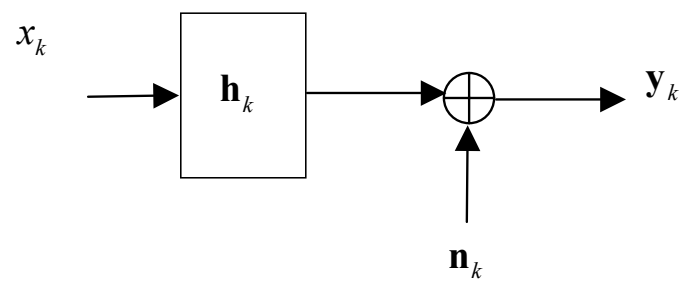

Figure 2.2. Discrete baseband signal model.

where $k$ is the time index, $l$ represents the over-sampling index,

$$
h_{k, l}^{i}=p(t) \circledast f^{i}(t) \circledast q(t)_{\mid t=k T+l \frac{T}{s}}
$$

and $n_{k, l}^{i}=n^{i}(t)_{\mid t=k T+l \frac{T}{s}}$. The ISI channel is assumed to be time limited to a length $(v+1)$. In the rest of the paper we adopt vector notation of [5] for representing the over-sampled outputs of multiple antenna signals. Then (2.4) can be compactly represented as

$$
\mathbf{y}_{k}=\mathbf{h}_{k} \circledast x_{k}+\mathbf{n}_{k},
$$

where we stack up over-sampled antenna outputs in a column vector format as:

$$
\begin{aligned}
& \mathbf{y}_{k}=\left[y_{k, 1}^{1}, . ., y_{k, s}^{1}, . ., y_{k, 1}^{N}, . ., y_{k, s}^{N}\right]^{\prime} \\
& \mathbf{h}_{k}=\left[h_{k, 1}^{1}, . ., h_{k, s}^{1}, . ., h_{k, 1}^{N}, . ., h_{k, s}^{N}\right]^{\prime} \\
& \mathbf{n}_{k}=\left[n_{k, 1}^{1}, . ., n_{k, s}^{1}, . ., n_{k, 1}^{N}, . ., n_{k, s}^{N}\right]^{\prime} .
\end{aligned}
$$

A schematic diagram of the above signal model is depicted in Figure 2.2.

\subsection{Signal Model with I-Q Split}

As shown in Figure 2.3, we extract multiple signal streams by extracting the in-phase (real) and quadrature (imaginary) components of the over-sampled outputs from each antenna. By stacking up the I-Q parts of (2.5) in a column vector format we get:

$$
\tilde{\mathbf{y}}_{k}=\sum_{l=0}^{v} \tilde{\mathbf{h}}_{l} x_{k-l}+\tilde{\mathbf{n}}_{k}, \quad P A M
$$




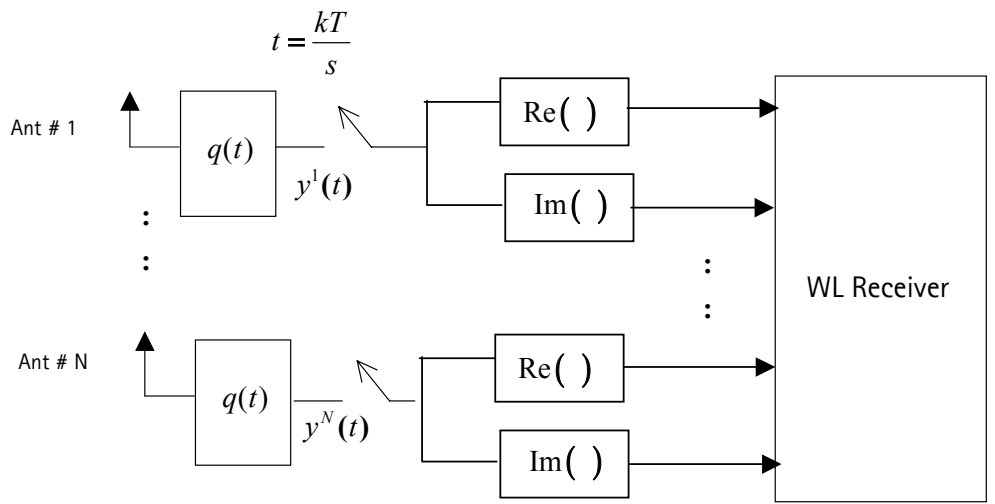

Figure 2.3. Signal model with I-Q split.

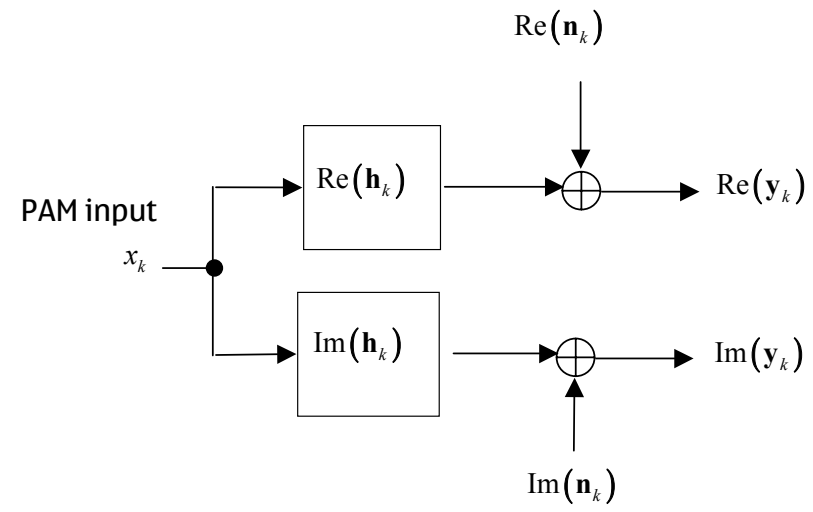

Figure 2.4. Equivalent baseband I-Q signal model for PAM.

where,

$$
\tilde{\mathbf{y}}_{k}=\left[\begin{array}{c}
\operatorname{Re}\left(\mathbf{y}_{k}\right) \\
\operatorname{Im}\left(\mathbf{y}_{k}\right)
\end{array}\right], \tilde{\mathbf{h}}_{l}=\left[\begin{array}{c}
\operatorname{Re}\left(\mathbf{h}_{l}\right) \\
\operatorname{Im}\left(\mathbf{h}_{l}\right)
\end{array}\right], \quad \tilde{\mathbf{n}}_{k}=\left[\begin{array}{c}
\operatorname{Re}\left(\mathbf{n}_{k}\right) \\
\operatorname{Im}\left(\mathbf{n}_{k}\right)
\end{array}\right]
$$

Since the modulation alphabet is real, I-Q split creates two copies of signal and noise with real valued channel gains. Thus, we have a single input (scalar PAM input) multiple output (SIMO) system model. A schematic diagram of above signal model for PAM signaling is shown in Figure 2.4. 


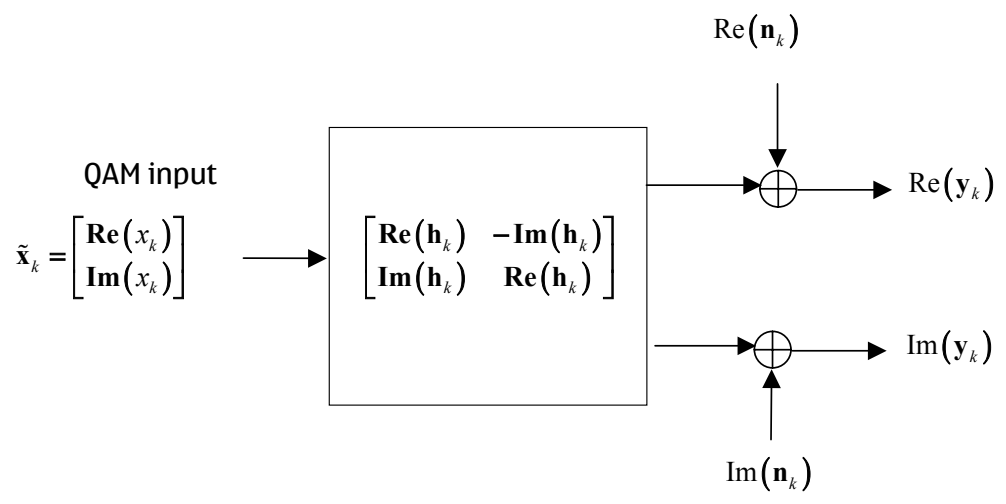

Figure 2.5. Equivalent baseband I-Q signal model for QAM.

For QAM signaling, since the modulation alphabets are complex quantities, and since the channel is assumed to be complex, I-Q split creates vector-matrix signal model of form:

$$
\tilde{\mathbf{y}}_{k}=\sum_{l=0}^{v} \tilde{\mathbf{H}}_{l} \tilde{\mathbf{x}}_{k-l}+\tilde{\mathbf{n}}_{k}, \quad Q A M
$$

where the channel takes a matrix form: $\tilde{\mathbf{H}}_{l}=\left[\begin{array}{cc}\operatorname{Re}\left(\mathbf{h}_{l}\right) & -\operatorname{Im}\left(\mathbf{h}_{l}\right) \\ \operatorname{Im}\left(\mathbf{h}_{l}\right) & \operatorname{Re}\left(\mathbf{h}_{l}\right)\end{array}\right]$ and the modulation takes a vector form: $\tilde{\mathbf{x}}_{k}=\left[\operatorname{Re}\left(x_{k}\right), \operatorname{Im}\left(x_{k}\right)\right]^{\prime}$. In this case, I-Q split creates a multiple input (constituted by I-Q parts of QAM) multiple output (MIMO) signal model. A schematic representation of the QAM signal model is given in Figure 2.5. The time domain signals given in $(2.5),(2.6),(2.7)$ can be represented in D-domain as:

$$
\begin{array}{rlrl}
\mathbf{y}(D) & =\mathbf{h}(D) x(D)+\mathbf{n}(D), & & \text { Conv } \\
\tilde{\mathbf{y}}(D) & =\tilde{\mathbf{h}}(D) x(D)+\tilde{\mathbf{n}}(D), & \text { PAM } \\
& =\tilde{\mathbf{H}}(D) \tilde{\mathbf{x}}(D)+\tilde{\mathbf{n}}(D) & \text { QAM. }
\end{array}
$$


Further, the D-domain channel vectors/matrices can be explicitly written as:

$$
\begin{aligned}
\mathbf{h}(D) & =\left[\mathbf{h}_{I}(D)+j \mathbf{h}_{Q}(D)\right] \\
\tilde{\mathbf{h}}(D) & =\left[\begin{array}{c}
\mathbf{h}_{I}(D) \\
\mathbf{h}_{Q}(D)
\end{array}\right]
\end{aligned}
$$

and

$$
\tilde{\mathbf{H}}(D)=\left[\begin{array}{cc}
\mathbf{h}_{I}(D) & -\mathbf{h}_{Q}(D) \\
\mathbf{h}_{Q}(D) & \mathbf{h}_{I}(D)
\end{array}\right]
$$

We further express the I-Q channel vectors as:

$$
\begin{aligned}
\mathbf{h}_{I}(D) & =\left[h_{I, 1}^{1}(D), . ., h_{I, s}^{1}(D), . ., h_{I, 1}^{N}(D), . ., h_{I, s}^{N}(D)\right]^{\prime} \\
\mathbf{h}_{Q}(D) & =\left[h_{Q, 1}^{1}(D), . ., h_{Q, s}^{1}(D), . ., h_{Q, 1}^{N}(D), . ., h_{Q, s}^{N}(D)\right]^{\prime}
\end{aligned}
$$

where $h_{p}^{q}(D), p=1,2, . ., s, q=1,2, . ., N$ being indices, denotes the D-transform of $h_{k, p}^{q}$ and $h_{I, p}^{q}(D), h_{Q, p}^{q}(D)$ denote the D-transform of real and imaginary parts of $h_{k, p}^{q}$. Further, we assume that the modulation symbols are identical and statistically independent (i.i.d.). In case of QAM, the I-Q parts of the symbol constellation are assumed to be zero mean uncorrelated symbols with equal variance. Then we have: $E\left[\left|x_{k}\right|^{2}\right]=1, E\left[\tilde{\mathbf{x}}_{k} \tilde{\mathbf{x}}_{k}^{\dagger}\right]=\frac{\mathbf{I}}{2}$.

\subsection{Interference Modeling}

We assume that the received signal is corrupted by a sum of $M$ co-channel interferers and thermal noise. The interference plus thermal noise term can be represented in discrete baseband form as

$$
\mathbf{n}_{k}=\sum_{m=1}^{M} \mathbf{g}_{k}^{m} \circledast \hat{x}_{k}^{m}+\mathbf{z}_{k}
$$


where

$$
\mathbf{g}_{k}^{m}=\left[g_{k, 1}^{1, m}, . ., g_{k, s}^{1, m}, . ., g_{k, 1}^{N, m}, . ., g_{k, s}^{N, m}\right]^{\prime}
$$

represents the channel vector of $m$ th interfering signal, $\hat{x}_{k}^{m}$ represents the $m$ th modulation sequence and

$$
\mathbf{z}_{k}=\left[z_{k, 1}^{1}, . ., z_{k, s}^{1}, . ., z_{k, 1}^{N}, . ., z_{k, s}^{N}\right]^{\prime}
$$

represents AWGN of variance $s N_{0}$ per dimension [5]. We assume that $\hat{x}_{k}^{m}$ are zero mean i.i.d. symbols that are statistically independent of desired modulation sequence, and $E\left|\hat{x}_{k}^{m}\right|^{2}=1$. Since the interference modulation alphabet is assumed to be PAM or QAM type, we assume that there are $M_{1}$ PAM interferers and $M_{2}$ QAM interferers in the system. With I-Q split, the noise plus interference term can be modeled as:

$$
\tilde{\mathbf{n}}_{k}=\underbrace{\sum_{l=1}^{M_{1}} \tilde{\mathbf{g}}_{\mathbf{k}}^{l} \circledast \hat{x}_{k}^{l}}_{\text {PAM CCI }}+\underbrace{\sum_{m=1}^{M_{2}} \tilde{\mathbf{G}}_{k}^{m} \circledast \overline{\mathbf{x}}_{k}^{m}}_{\text {QAM CCI }}+\underbrace{\tilde{\mathbf{z}}_{k}}_{\text {AWGN }}
$$

where

$$
\tilde{\mathbf{g}}_{\mathbf{k}}^{l}=\left[\operatorname{Re}\left(\mathbf{g}_{k}^{l}\right), \operatorname{Im}\left(\mathbf{g}_{k}^{l}\right)\right]^{\prime}, \quad \tilde{\mathbf{G}}_{k}^{m}=\left[\begin{array}{cc}
\operatorname{Re}\left(\mathbf{g}_{k}^{m}\right) & -\operatorname{Im}\left(\mathbf{g}_{k}^{m}\right) \\
\operatorname{Im}\left(\mathbf{g}_{k}^{m}\right) & \operatorname{Re}\left(\mathbf{g}_{k}^{m}\right)
\end{array}\right],
$$

$\tilde{\mathbf{z}}_{k}=\left[\operatorname{Re}\left(\mathbf{z}_{k}\right), \operatorname{Im}\left(\mathbf{z}_{k}\right)\right]^{\prime}$ denotes the real and imaginary parts of thermal noise of variance $\frac{s N_{0}}{2}$ per dimension, and $\overline{\mathbf{x}}_{k}^{m}=\left[\operatorname{Re}\left(\hat{x}_{k}^{m}\right), \operatorname{Im}\left(\hat{x}_{k}^{m}\right)\right]^{\prime}$. We assume that $E\left[\left|\hat{x}_{k}^{l}\right|^{2}\right]=1$, $E\left[\overline{\mathbf{x}}_{k}^{m} \overline{\mathbf{x}}_{k}^{\dagger m}\right]=\frac{\mathbf{I}}{2}$. Taking the D-transforms of (2.13), (2.14), the time domain signals can be expressed in D-domain as:

$$
\begin{aligned}
\mathbf{n}(D) & =\sum_{l=1}^{M} \mathbf{g}^{l}(D) \hat{x}^{l}(D)+\mathbf{z}(D) \\
\tilde{\mathbf{n}}(D) & =\sum_{l=1}^{M_{1}} \tilde{\mathbf{g}}^{l}(D) \hat{x}^{l}(D)+\sum_{m=1}^{M_{2}} \tilde{\mathbf{G}}^{m}(D) \overline{\mathbf{x}}^{m}(D)+\tilde{\mathbf{z}}(D) .
\end{aligned}
$$

We use the following notation to explicitly represent the above D-domain vectors

$$
\mathbf{g}^{l}(D)=\left[g_{1}^{1, l}(D), . ., g_{s}^{1, l}(D), . ., g_{s}^{N, l}(D)\right]^{\prime}
$$




$$
\tilde{\mathbf{g}}^{l}(D)=\left[\begin{array}{c}
\mathbf{g}_{I}^{l}(D) \\
\mathbf{g}_{Q}^{l}(D)
\end{array}\right]
$$

and

$$
\tilde{\mathbf{G}}^{m}(D)=\left[\begin{array}{cc}
\mathbf{g}_{I}^{l}(D) & -\mathbf{g}_{Q}^{l}(D) \\
\mathbf{g}_{Q}^{l}(D) & \mathbf{g}_{I}^{l}(D)
\end{array}\right]
$$

where

$$
\begin{aligned}
\mathbf{g}_{I}^{l}(D) & =\left[g_{I, 1}^{1, l}(D), . ., g_{I, s}^{1, l}(D), . ., g_{I, s}^{N, l}(D)\right]^{\prime} \\
\mathbf{g}_{Q}^{l}(D) & =\left[g_{Q, 1}^{1, l}(D), . ., g_{Q, s}^{1, l}(D), . ., g_{Q, s}^{N, l}(D)\right]^{\prime}
\end{aligned}
$$

where $g_{q}^{r, s}(D)$ and $g_{q}^{r, s}(D), q, r, s$ being indices denotes the D-transform of $g_{k, q}^{r, s}$, and $g_{I, q}^{r, s}(D), g_{Q, q}^{r, s}(D)$ denotes the D-transform of real and imaginary parts of $g_{k, q}^{r, s}$ respectively. Using (2.15), the conventional noise plus interference correlation matrix (NCM) is given by:

$$
\begin{aligned}
\mathbf{R}_{\mathbf{n n}}(D) & \triangleq E\left[\mathbf{n}(D) \mathbf{n}^{\dagger}\left(D^{-*}\right)\right] \\
& =\sum_{m=1}^{M} \mathbf{g}^{m}(D) \mathbf{g}^{m \dagger}\left(D^{-*}\right)+s N_{0} \mathbf{I} .
\end{aligned}
$$

For convenience let us introduce: $\mathbf{R}_{\mathbf{n n}}(D) \triangleq \mathbf{R}_{\mathbf{i i}}(D)+s N_{0} \mathbf{I}$ where

$$
\mathbf{R}_{\mathbf{i i}}(D) \triangleq \sum_{m=1}^{M} \mathbf{g}^{m}(D) \mathbf{g}^{m \dagger}\left(D^{-*}\right)
$$

denotes the interference correlation matrix (ICM). Further, using (2.16), we define the NCM of the I-Q signal as:

$$
\begin{aligned}
\mathbf{R}_{\tilde{\mathbf{n}} \tilde{\mathbf{n}}}(D) & \triangleq E\left[\tilde{\mathbf{n}}(D) \tilde{\mathbf{n}}^{\dagger}\left(D^{-*}\right)\right] \\
& =\mathbf{R}_{\tilde{\mathbf{i i}}}(D)+\frac{s N_{0}}{2} \mathbf{I}
\end{aligned}
$$


where

$$
\mathbf{R}_{\widetilde{\mathbf{i i}}}(D) \triangleq \underbrace{\sum_{l=1}^{M_{1}} \tilde{\mathbf{g}}^{l}(D) \tilde{\mathbf{g}}^{l \dagger}\left(D^{-*}\right)}_{\text {PAM CCI contribution }}+\underbrace{\frac{1}{2} \sum_{m=1}^{M_{2}} \tilde{\mathbf{G}}^{m}(D) \tilde{\mathbf{G}}^{m \dagger}\left(D^{-*}\right)}_{\text {QAM CCI contribution }}
$$

denotes the I-Q interference correlation matrix (ICM). 


\section{CHAPTER 3}

\section{WIDELY LINEAR EQUALIZATION FOR PAM SYSTEMS}

\subsection{Introduction}

It is well known that conventional MMSE equalizers suffer from noise enhancement in channels with zeros near unit circle. In [28] Gerstacket et al. showed that WL equalizers that independently filter the complex, complex-conjugate (or the I-Q) parts of the signal reduce the noise enhancement problem as WLE are less likely to experience channel zeros near unit circle. In [28], the authors mainly relied on simulation to show the advantage of WL receivers in frequency selective channels. We take a more theoretical approach. In this chapter we show that the advantage of WL methods can be explained using certain MSE connection between conventional and WL receivers. We provide new insights into the performance and computational complexity of conventional and WL equalizers using detailed MSE analysis.

Intentionally, we depart from the customary complex, complex-conjugate based WL approach and formulate WL MMSE/MMSE-DFE equalizers using the in-phase (I) and quadrature (Q) parts of the received signal. We consider both finite and infinite length equalizers. Although our focus in this chapter is restricted to a single antenna receiver $(N=1)$ in a white noise channel, we keep the derivations general enough to include extension to multiple antennas and non-white noise cases.

The chapter is organized as follows. In Section 3.2 we analyze conventional and WL linear equalizers first for infinite length case followed by finite length scenario. In 
Section 3.3 we provide analogous results for DFE case followed by a summary of the results of this chapter in Section 3.4.

\subsection{Linear Equalization}

First we shall begin with the well-known conventional LE. The notation and derivations for infinite length equalizers are obtained from [5] while derivations for finite length case are obtained from [39]. Although our results are self-contained, the interested reader may refer to the references for additional details.

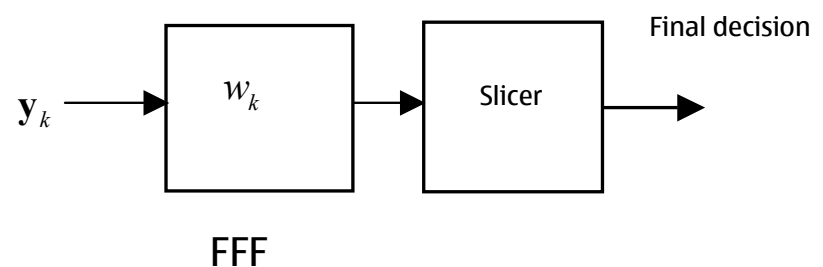

Figure 3.1. Conventional linear equalizer.

\subsubsection{Infinite Length Conventional Linear Equalizer}

A standard linear equalizer as depicted in Figure 3.1 uses a vector-valued linear feedforward (FFF) filter

$$
\mathbf{w}_{k}=\left[w_{1, k}, w_{2, k}, . ., w_{N s, k}\right]
$$

which convolves with the over-sampled data vector (introduced in (2.5)):

$$
\mathbf{y}_{k}=\left[y_{1, k}, y_{2, k}, . ., y_{N s, k}\right]^{\prime}
$$

that produces a scalar output sequence $z_{k}=\mathbf{w}_{k} \circledast \mathbf{y}_{k}$ to provide an MMSE estimate of $x_{k}$. Denoting the error sequence by $e_{k}$ we have:

$$
e_{k} \triangleq x_{k}-z_{k}
$$


We seek the optimum filter that minimizes the mean square error (MSE) metric:

$$
\mathrm{MSE}_{\mathrm{Conv} \mathrm{LE}} \triangleq \min _{\mathbf{w}_{k}} E\left[\left|x_{k}-z_{k}\right|^{2}\right]
$$

Using D-notation:

$$
e(D)=x(D)-\mathbf{w}(D) \mathbf{y}(D)
$$

Using orthogonality principle:

$$
E\left[e(D) \mathbf{y}^{\dagger}\left(D^{-*}\right)\right]=\mathbf{R}_{\mathbf{x y}}(D)-\mathbf{w}(D) \mathbf{R}_{\mathbf{y y}}(D)=0
$$

together with (3.1) we can obtain the optimum FFF as:

$$
\begin{aligned}
\mathbf{w}(D) & =\mathbf{R}_{\mathbf{x y}}(D) \mathbf{R}_{\mathbf{y y}}^{-\mathbf{1}}(D) \\
& =R_{x x}(D) \mathbf{h}^{\dagger}\left(D^{-*}\right)\left[\mathbf{h}(D) \mathbf{h}^{\dagger}\left(D^{-*}\right)+\mathbf{R}_{\mathbf{n n}}(D)\right]^{-1} \\
& =\left[R_{x x}^{-1}(D)+\mathbf{h}^{\dagger}\left(D^{-*}\right) \mathbf{R}_{\mathbf{n n}}^{-\mathbf{1}}(D) \mathbf{h}(D)\right]^{-1} \mathbf{h}^{\dagger}\left(D^{-*}\right) \mathbf{R}_{\mathbf{n n}}^{-\mathbf{1}}(D) .
\end{aligned}
$$

In the above expressions (3.4) is obtained by applying matrix inversion lemma ${ }^{1}$ and

$$
\begin{aligned}
\mathbf{R}_{\mathbf{x y}}(D) \triangleq E\left[x(D) \mathbf{y}^{\dagger}\left(D^{-*}\right)\right]=R_{x x}(D) \mathbf{h}^{\dagger}\left(D^{-*}\right) \\
\mathbf{R}_{\mathbf{y} \mathbf{y}}(D) \triangleq E\left[\mathbf{y}(D) \mathbf{y}^{\dagger}\left(D^{-*}\right)\right]=\mathbf{h}(D) \mathbf{h}^{\dagger}\left(D^{-*}\right)+\mathbf{R}_{\mathbf{n n}}(D) \\
\mathbf{R}_{\mathbf{n n}}(D) \triangleq E\left[\mathbf{n}(D) \mathbf{n}^{\dagger}\left(D^{-*}\right)\right] \\
R_{x x}(D) \triangleq E\left[x(D) x^{*}\left(D^{-*}\right)\right]=1
\end{aligned}
$$

The minimum MSE for this case can be obtained as [5]:

$$
\mathrm{MSE}_{\text {Conv LE }}=T \int_{-\frac{1}{2 T}}^{\frac{1}{2 T}} \frac{1}{\left[1+\mathbf{h}^{\dagger}(-f) \mathbf{R}_{\mathbf{n n}}^{-1}(f) \mathbf{h}(f)\right]} d f
$$

where frequency domain quantities are used in defining the MSE. In case of white noise, $\mathbf{R}_{\mathbf{n n}}(D)=s N_{0} \mathbf{I}$. Hence we can represent the FFF alternatively as:

$$
\mathbf{w}(D)=\underbrace{\frac{1}{\left[s N_{0}+\mathbf{h}^{\dagger}\left(D^{-*}\right) \mathbf{h}(D)\right]}}_{\text {scalar MMSE filter }} \underbrace{\mathbf{h}^{\dagger}\left(D^{-*}\right)}_{\text {Matched Filter }} .
$$

$$
{ }^{1}(A+B C D)^{-1}=A^{-1}-A^{-1} B\left(D A^{-1} B+C^{-1}\right)^{-1} D A^{-1}
$$


Here, the FFF filter is split into a matched filter and a scalar MMSE whitening filter. Note that the alternative form is computationally more efficient than the direct form (3.3) as this implementation requires a single scalar MMSE filter after applying a vector-valued matched filter (MF) filter. Next we shall examine the structure and MSE performance for an infinite length WL-LE.

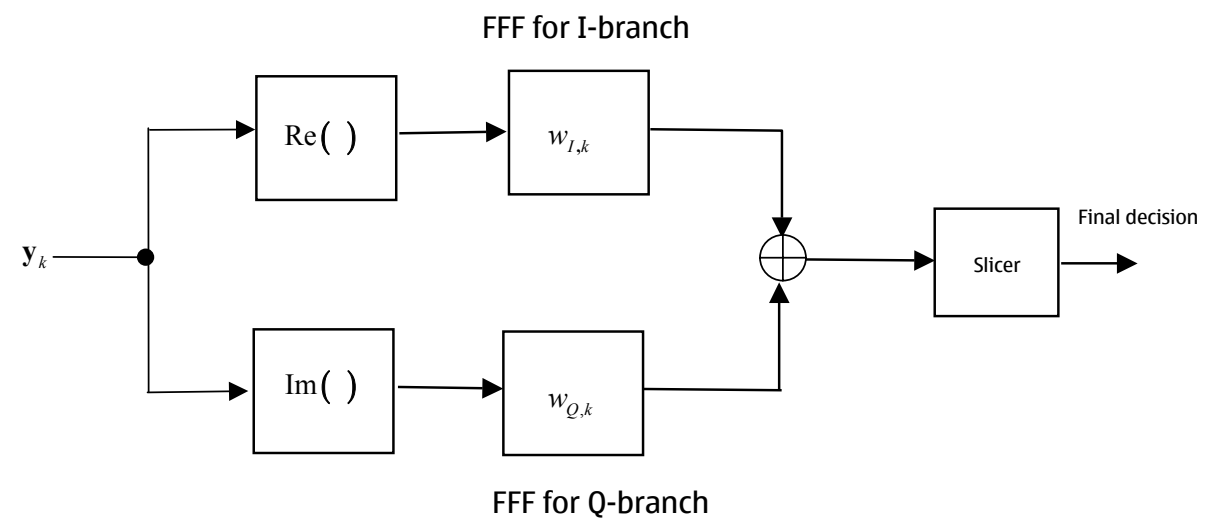

Figure 3.2. WL linear equalizer for PAM.

\subsubsection{Infinite Length WL Linear Equalizer}

In contrast to the conventional LE, the WL-LE independently filters the I/Q data branches (see Figure 3.2) and combines these outputs to form a decision variable:

$$
\tilde{z}_{k}=\tilde{\mathbf{w}}_{k} \circledast \tilde{\mathbf{y}}_{k} .
$$

In the above expression, the WL filters, compactly denoted as:

$$
\tilde{\mathbf{w}}_{k}=\left[\mathbf{w}_{I, k}, \mathbf{w}_{Q, k}\right]
$$

are optimized by minimizing the MSE metric:

$$
\mathrm{MSE}_{\mathrm{WL} \text { LE }} \triangleq \min _{\tilde{\mathbf{w}}_{k}} E\left[\left|x_{k}-\tilde{z}_{k}\right|^{2}\right]
$$


Following the results of conventional LE, we can obtain the D-domain solution for WL-LE as:

$$
\begin{aligned}
\tilde{\mathbf{w}}(D) & =\mathbf{R}_{\tilde{\mathbf{x}} \tilde{\mathbf{y}}}(D) \mathbf{R}_{\tilde{\mathbf{y}} \tilde{\mathbf{y}}}^{-1}(D) \\
& =R_{x x}(D) \tilde{\mathbf{h}}^{\dagger}\left(D^{-*}\right)\left[\tilde{\mathbf{h}}(D) \tilde{\mathbf{h}}^{\dagger}\left(D^{-*}\right)+\mathbf{R}_{\tilde{\mathbf{n}} \tilde{\mathbf{n}}}(D)\right]^{-1} \\
& =\left[R_{x x}^{-1}(D)+\tilde{\mathbf{h}}^{\dagger}\left(D^{-*}\right) \mathbf{R}_{\tilde{\mathbf{n}} \tilde{\mathbf{n}}}^{-1}(D) \tilde{\mathbf{h}}(D)\right]^{-1} \tilde{\mathbf{h}}^{\dagger}\left(D^{-*}\right) \mathbf{R}_{\tilde{\mathbf{n}} \tilde{\mathbf{n}}}^{-1}(D)
\end{aligned}
$$

where

$$
\begin{aligned}
& \mathbf{R}_{\tilde{\mathbf{x}} \tilde{\mathbf{y}}}(D) \triangleq E\left[x(D) \tilde{\mathbf{y}}^{\dagger}\left(D^{-*}\right)\right]=R_{x x}(D) \tilde{\mathbf{h}}^{\dagger}\left(D^{-*}\right) \\
& \mathbf{R}_{\tilde{\mathbf{y}} \tilde{\mathbf{y}}}(D) \triangleq E\left[\tilde{\mathbf{y}}(D) \tilde{\mathbf{y}}^{\dagger}\left(D^{-*}\right)\right]=\tilde{\mathbf{h}}(D) \tilde{\mathbf{h}}^{\dagger}\left(D^{-*}\right)+\mathbf{R}_{\tilde{\mathbf{n}} \tilde{\mathbf{n}}}(D) \\
& \mathbf{R}_{\tilde{\mathbf{n}} \tilde{\mathbf{n}}(D)} \triangleq E\left[\tilde{\mathbf{n}}(D) \tilde{\mathbf{n}}^{\dagger}\left(D^{-*}\right)\right]
\end{aligned}
$$

The minimum MSE in this case is given by:

$$
\mathrm{MSE}_{\mathrm{WL} L \mathrm{LE}}=T \int_{-\frac{1}{2 T}}^{\frac{1}{2 T}} \frac{1}{\left[1+\tilde{\mathbf{h}}^{\dagger}(-f) \mathbf{R}_{\tilde{\mathbf{n}} \tilde{\mathbf{n}}}^{-1}(f) \tilde{\mathbf{h}}(f)\right]} d f .
$$

In case of white noise, $\mathbf{R}_{\tilde{\mathbf{n}} \tilde{\mathbf{n}}}(D)=\frac{s N_{0}}{2} \mathbf{I}$. Using this, the WL-FFF can be expressed as:

$$
\mathbf{w}_{I}(D)=\frac{\mathbf{h}_{I}^{\dagger}\left(D^{-*}\right)}{\left[\frac{s N_{0}}{2}+\tilde{\mathbf{h}}^{\dagger}\left(D^{-*}\right) \tilde{\mathbf{h}}(D)\right]}, \quad \mathbf{w}_{Q}(D)=\frac{\mathbf{h}_{Q}^{\dagger}\left(D^{-*}\right)}{\left[\frac{s N_{0}}{2}+\tilde{\mathbf{h}}^{\dagger}\left(D^{-*}\right) \tilde{\mathbf{h}}(D)\right]} .
$$

Note that in this case the WL FFF is a real-valued vector filter of length $2 s N$ that is twice that of conventional filter. In spite of increase in filter length, in Section 3.2.4, we show that the overall complexity of WL receiver is lower than that of a conventional receiver when the noise is white.

\subsubsection{MSE Comparison for Infinite Length LE}

In this section, we highlight the advantage of WL detection by using a closed form expression that shows the MSE connection between conventional and WL receivers. Let

$$
Q(D) \triangleq \mathbf{h}^{\dagger}\left(D^{-*}\right) \mathbf{h}(D)+\frac{s N_{0}}{2}
$$


denote the covariance function for conventional case. Strictly speaking, the covariance function for conventional signal should be defined as: $\mathbf{h}^{\dagger}\left(D^{-*}\right) \mathbf{h}(D)+s N_{0}$ for complex AWGN input. Since, only the real part of the noise contributes to the performance, we modify the covariance definition to include $\frac{s N_{0}}{2}$ as noise variance. Such a modification allows us to draw a direct comparison between conventional and WL methods ${ }^{2}$. Using, (2.8), (2.11), (2.12), we can express $Q(D)$ in terms of respective I/Q channel responses as:

$$
\begin{aligned}
Q(D) & =\mathbf{h}^{\dagger}\left(D^{-*}\right) \mathbf{h}(D)+\frac{s N_{0}}{2} \\
& =\left[\mathbf{h}_{I}^{\dagger}\left(D^{-*}\right)-j \mathbf{h}_{Q}^{\dagger}\left(D^{-*}\right)\right]\left[\mathbf{h}_{I}(D)+j \mathbf{h}_{Q}(D)\right]+\frac{s N_{0}}{2} \\
& =\sum_{l=1}^{s} \sum_{m=1}^{N}\left[\left|h_{I, l}^{m}(D)\right|^{2}+\left|h_{Q, l}^{m}(D)\right|^{2}+2 \operatorname{Im}\left[h_{Q, l}^{m}\left(D^{-*}\right) h_{I, l}^{m}(D)\right]\right]+\frac{s N_{0}}{2}
\end{aligned}
$$

Letting $D=e^{-j 2 \pi f T}$ we get:

$$
\begin{aligned}
Q(f) & =\sum_{l=1}^{s} \sum_{m=1}^{N}\left[\left|h_{I, l}^{m}(f)\right|^{2}+\left|h_{Q, l}^{m}(f)\right|^{2}+2 \operatorname{Im}\left[h_{Q, l}^{m}(-f) h_{I, l}^{m}(f)\right]\right]+s \frac{N_{0}}{2} \\
& =a(f)-\bar{a}(f)
\end{aligned}
$$

where

$$
\begin{aligned}
& a(f) \triangleq \sum_{l=1}^{s} \sum_{m=1}^{N}\left[\left|h_{I, l}^{m}(f)\right|^{2}+\left|h_{Q, l}^{m}(f)\right|^{2}\right]+s \frac{N_{0}}{2} \\
& \bar{a}(f) \triangleq 2 \sum_{l=1}^{s} \sum_{m=1}^{N} \operatorname{Im}\left[h_{Q, l}^{m}(-f) h_{I, l}^{m}(f)\right] .
\end{aligned}
$$

We can verify that $a(f)$ is an even function of $f$ and $\bar{a}(f)$ is a real valued odd function i.e. $\int_{-\frac{1}{2 T}}^{\frac{1}{2 T}} \bar{a}(f) d f=0$. Using $(2.11),(2.12)$ let us define:

$$
\tilde{Q}(f) \triangleq \tilde{\mathbf{h}}^{\dagger}(-f) \tilde{\mathbf{h}}(f)+\frac{s N_{0}}{2}=\sum_{l=1}^{s} \sum_{m=1}^{N}\left[\left|h_{I, l}^{m}(f)\right|^{2}+\left|h_{Q, l}^{m}(f)\right|^{2}\right]+\frac{s N_{0}}{2}=a(f) .
$$

\footnotetext{
${ }^{2}$ Alternatively, we could let the noise variance be $s N_{0}$ and compare the MSE for conventional and WL cases in the limiting case when $N_{0}$ takes a zero value.
} 
For a non zero constant noise variance $N_{0}$ we have:

$$
Q(f)=a(f)-\bar{a}(f)>0 \Longrightarrow \frac{\bar{a}(f)}{a(f)}<1 .
$$

Using (3.9),(3.16) the MSE for a WL-LE can be expressed as:

$$
\begin{aligned}
\text { MSE }_{\text {WL LE }} & =T \frac{s N_{0}}{2} \int_{-\frac{1}{2 T}}^{\frac{1}{2 T}} \frac{1}{\tilde{\mathbf{h}}^{\dagger}(-f) \tilde{\mathbf{h}}(f)+s \frac{N_{0}}{2}} d f \\
& =T \frac{s N_{0}}{2} \int_{-\frac{1}{2 T}}^{\frac{1}{2 T}} \frac{1}{a(f)} d f .
\end{aligned}
$$

Using (3.5),(3.13) the MSE for a conventional equalizer can be expressed $\mathrm{as}^{3}$ :

$$
\begin{aligned}
\text { MSE }_{\text {Conv LE }} & =T \frac{s N_{0}}{2} \int_{-\frac{1}{2 T}}^{\frac{1}{2 T}} \frac{1}{\mathbf{h}^{\dagger}(-f) \mathbf{h}(f)+\frac{s N_{0}}{2}} d f \\
& =T \frac{s N_{0}}{2} \int_{-\frac{1}{2 T}}^{\frac{1}{2 T}} \frac{1}{a(f)-\bar{a}(f)} d f \\
& =T \frac{s N_{0}}{2} \int_{-\frac{1}{2 T}}^{\frac{1}{2 T}} \frac{1}{a(f)\left[1-\frac{\bar{a}(f)}{a(f)}\right]} d f \\
& =T \frac{s N_{0}}{2} \int_{-\frac{1}{2 T}}^{\frac{1}{2 T}} \frac{1}{a(f)}\left[1+\frac{\bar{a}(f)}{a(f)}+\frac{\bar{a}^{2}(f)}{a^{2}(f)}+\frac{\bar{a}^{3}(f)}{a^{3}(f)}+. .\right] d f \\
& =T \frac{s N_{0}}{2} \int_{-\frac{1}{2 T}}^{\frac{1}{2 T}}\left[\frac{1}{a(f)}+\frac{\frac{\bar{a}^{2}(f)}{a^{2}(f)}+\frac{\bar{a}^{4}(f)}{a^{4}(f)}+. .}{a(f)}\right] d f \\
& =\operatorname{MSE}_{\text {WL LE }}+\operatorname{Excess} \operatorname{MSE} .
\end{aligned}
$$

In the above expressions we invoked the infinite series of type $[1-x]^{-1}=\sum_{k=0}^{\infty} x^{k}$ and eliminated the terms containing odd-powers as they integrate to zero. The excess MSE is a non-negative quantity since $a(f)$ and the even powers of $\frac{\bar{a}(f)}{a(f)}$ are always positive. Now we have the desired result. The MSE for WL-LE is less than or equal to conventional MSE and equality holds when the excess MSE term is zero, which happens if only if $\bar{a}(f)=0$ i.e. $-2 \sum_{l=1}^{s} \operatorname{Im}\left[h_{Q, l}(-f) h_{I, l}(f)\right]=0$. This condition will

\footnotetext{
${ }^{3}$ Note that for PAM, only the real part of noise contributes to error rate. Therefore we divide the MSE expression given by (3.5) by a factor 2 .
} 
be satisfied when all channel taps are real or when the channel is ISI free. In all other complex valued ISI channels WL-LE provides a lower MSE than conventional LE. Alternatively, we can say that the presence of $\bar{a}(f)$ (that is caused by the imaginary part of time domain signal covariance) is responsible for additional noise enhancement which is quantified by the excess MSE term. This problem is most severe for channels that has a large $\bar{a}(f)$. We further emphasize this result using the following examples.

\section{Example-1}

Consider linear equalization of a binary modulation alphabet over a single antenna 2tap ISI channel whose impulse response is given by $h_{k}=[0.707, j 0.707]$. In frequency domain: $\mathbf{h}(f)=0.707\left(1+j e^{j 2 \pi f T}\right)$. Assuming white Gaussian noise of variance $\frac{N_{0}}{2}$ per dimension, the MSE for conventional LE is given by:

$$
\operatorname{MSE}_{\text {Conv }}=\frac{N_{0} T}{2} \int_{\frac{-1}{2 T}}^{\frac{1}{2 T}} \frac{1}{|\mathbf{h}(f)|^{2}+\frac{N_{0}}{2}} d f=\frac{N_{0} T}{2} \int_{\frac{-1}{2 T}}^{\frac{1}{2 T}} \frac{1}{1-\sin (2 \pi f T)+\frac{N_{0}}{2}} d f .
$$

For $\mathrm{SNR}=\frac{1}{N_{0}}=10 \mathrm{~dB}$, using numerical integration we get: $\mathrm{MSE}_{\mathrm{Conv}} \sim 0.156$. Since the unbiased SNR is calculated as:

$$
\mathrm{SNR}_{\mathrm{U}, \mathrm{Conv}}=10 \log \left[\frac{1}{\mathrm{MSE}_{\mathrm{Conv}}}-1\right]
$$

we get: $\operatorname{SNR}_{\mathrm{U}, \mathrm{Conv}}=7.332 \mathrm{~dB}$. On the other hand, for the WL-LE, we have:

$$
h_{I}(f)=0.707, \quad h_{Q}(f)=0.707 e^{j 2 \pi f T}
$$

Since

$$
\left|h_{I}(f)\right|^{2}+\left|h_{Q}(f)\right|^{2}=1
$$

the MSE becomes MSE $E_{\mathrm{WL}} \mathrm{LE}=\frac{N_{0}}{1+N_{0}}$. The unbiased SNR in this case is equal to 13 $\mathrm{dB}$ which is equal to the optimum matched filter bound (MFB). In contrast, the conventional equalizer suffers from high noise enhancement due to the presence of spectral null at $f=\frac{1}{4 T}$. This example clearly illustrates the superiority of WL framework for PAM signaling. 
Example-2

Next we evaluate performance of Example 3.5.2 from [5]. The channel taps are given by $h_{k}=[-0.5,1+j 0.25,-0.5]$. We normalize the channel profile to unit power and compute the MSE for $\mathrm{SNR}=10 \mathrm{~dB}$. In this case the conventional LE gives $\mathrm{MSE}_{\mathrm{Conv}} \sim 0.147$ whose unbiased SNR is equal to $\mathrm{SNR}_{\mathrm{U}, \mathrm{Conv}}=7.57 \mathrm{~dB}$ which corresponds to $5.36 \mathrm{~dB}$ loss with respect to (w.r.t) MFB. On the other hand the WL-LE gives $\mathrm{MSE}_{\mathrm{WL} L \mathrm{LE}} \sim 0.11, \mathrm{SNR}_{\mathrm{U}, \mathrm{WL} \mathrm{LE}}=9.08 \mathrm{~dB}$, a gain of $1.51 \mathrm{~dB}$ compared to conventional LE.

\subsubsection{Complexity Comparison}

In this section we highlight the complexity differences between conventional and WL methods for infinite length LE implementation. In Section 3.2.3, we have shown that WL receiver generally provides lower noise enhancement and a lower MSE. We will next show that lower noise enhancement also implies a computational advantage. In LE implementation, most of the complexity arises from the covariance inversion (MMSE filtering) step. Using D-notation, using (3.13), (3.16) let:

$$
a(D)-\bar{a}(D)=\frac{s N_{0}}{2}+\mathbf{h}^{\dagger}\left(D^{-*}\right) \mathbf{h}(D), \quad a(D)=\frac{s N_{0}}{2}+\tilde{\mathbf{h}}^{\dagger}\left(D^{-*}\right) \tilde{\mathbf{h}}(D)
$$

where we replaced $a(f), \bar{a}(f)$ with $a(D), \bar{a}(D)$. In case of white noise, using (3.4), (3.10) the FFF can be expressed as:

$$
\begin{aligned}
& \mathbf{w}(D)=\underbrace{\left[s \frac{N_{0}}{2}+\mathbf{h}^{\dagger}\left(D^{-*}\right) \mathbf{h}(D)\right]^{-1}}_{\text {MMSE section }} \underbrace{\mathbf{h}^{\dagger}\left(D^{-*}\right)}_{\text {MF }}=\frac{1}{[a(D)-b(D)]} \mathbf{h}^{\dagger}\left(D^{-*}\right) \\
& \tilde{\mathbf{w}}(D)=\underbrace{\left[s \frac{N_{0}}{2}+\tilde{\mathbf{h}}^{\dagger}\left(D^{-*}\right) \tilde{\mathbf{h}}(D)\right]^{-1}}_{\text {WL MMSE section }} \underbrace{\tilde{\mathbf{h}}^{\dagger}\left(D^{-*}\right)}_{\text {I-Q MF }}=\frac{1}{a(D)} \tilde{\mathbf{h}}^{\dagger}\left(D^{-*}\right) .
\end{aligned}
$$

Note that the MMSE section is a scalar filter whereas the MF is a vector. To fully understand the complexity differences between the two methods we analyze the implementation complexity for MF, MMSE filters separately. 


\subsubsection{Matched Filter (MF) Implementation}

In (3.20), the WL receiver has two sets of real-valued matched filters, one to match the real part and the other for the imaginary part, whereas in (3.19) conventional LE needs a single (complex valued) vector matched filter. Since $\tilde{\mathbf{h}}^{\dagger}\left(D^{-*}\right)$ is vector of length $2 s N$, the WL receiver needs $2 s N$ convolutions (between real signals) operations whereas the conventional MMSE receiver needs $s N$ complex valued convolutions (because $\mathbf{h}^{\dagger}\left(D^{-*}\right)$ is a row vector of length $\left.s N\right)$ which is equivalent to $4 s N$ real convolutions. Clearly, the conventional MF is more complex to implement than WL MF.

\subsubsection{MMSE Filter Implementation}

Next we consider the complexity differences in implementing the MMSE filter. The MMSE section for the conventional case can be expressed as:

$$
w_{\text {conv MMSE }}(D)=\frac{1}{a(D)}\left[1-\frac{\bar{a}(D)}{a(D)}\right]^{-1}
$$

whereas the MMSE section for WL case is given by $\frac{1}{a(D)}$. We see that conventional MMSE section requires an additional filter $\left[1-\frac{\bar{a}(D)}{a(D)}\right]^{-1}$. In time domain, the impulse response of the MMSE filter can be expressed as:

$$
w_{\text {Conv }}, \operatorname{MMSE}, k=\hat{a}_{k} \circledast \delta_{k}, k \in[-\infty, \infty]
$$

where $\hat{a}_{k} \leftrightarrow \frac{1}{a(D)}, \delta_{k} \leftrightarrow\left[1-\frac{\bar{a}(D)}{a(D)}\right]^{-1}$. For the WL case, the time domain MMSE response can be shown to be

$$
\tilde{w}_{\text {wL MMSE }, k}=\hat{a}_{k}, k \in[-\infty, \infty] .
$$

Note that $\hat{a}_{k}$ is a real-valued filter (by definition) whereas $\delta_{k}$ takes complex values. Let us assume that a truncated sequence of length $N_{f}$ closely approximates the WL MMSE filter. Because of convolution operation, $\delta_{k}$, lengthens the impulse response of 
$\tilde{w}_{\text {WL MMSE, } k}$. If $\delta_{k}$ has significant energy outside this truncation window, the conventional MMSE receiver has to consider a longer FFF filter. Therefore we can say that WL implementation has computational advantage in finite length implementation as well. We use the following example to further highlight the complexity differences between the two methods.

Example We analyze the implementation complexity of a LE in a single antenna system with a 2 -tap channel $\mathbf{h}(D)=1+j a D$ where $a$ is a positive constant less than 1. For $N_{0} \rightarrow 0$, the FFF can be approximated as:

$$
\mathbf{w}_{\mathrm{LE}}(D)=\left[N_{0}+\mathbf{h}^{\dagger}\left(D^{-*}\right) \mathbf{h}(D)\right]^{-1} \mathbf{h}^{\dagger}\left(D^{-*}\right) \sim \frac{1}{\mathbf{h}(D)}=\frac{1}{1+j a D} .
$$

We can express this filter in infinite series form:

$$
\mathbf{w}_{\mathrm{LE}}(D) \sim[1+j a D]^{-1}=\sum_{k=0}^{\infty}(-j a)^{k} D^{k} .
$$

The corresponding time domain sequence is given by

$$
w_{k}=(-j a)^{k}, k \geq 0
$$

For small values of $a$ the FFF can be truncated to the first few taps. However as $a \rightarrow 1$, the number of taps required to approach infinite length performance becomes large. In contrast the WL LE takes the form:

$$
\tilde{w}_{\mathrm{WL} \mathrm{LE}}(D)=\left[N_{0}+1+a^{2}\right]^{-1}\left[1, a D^{-1}\right]
$$

In this case, WL FFF reduces to a single scalar multiplication whereas the MF operation requires a single delay operation and two real multiplications only. In this example, I-Q split makes the system ISI free even before applying the MF.

\subsubsection{Finite Length Conventional Linear Equalizer}

Having demonstrated the superiority of WL approach in the infinite length case we turn our attention to the more practical finite length situation. We first formulate 
the conventional and WL-LE's using finite length filter constraints and analyze MSE performance to understand the differences. Recall that the discrete-time complexvalued signal model (2.5) is given by:

$$
\mathbf{y}_{k}=\mathbf{h}_{k} \circledast x_{k}+\mathbf{n}_{k}=\sum_{m=0}^{v} \mathbf{h}_{m} x_{k-m}+\mathbf{n}_{k}
$$

where we stacked up the over-sampled elements in vector format. Using notation from [39], we stack a block $N_{f}$ of output received elements $\mathbf{y}_{k}$ as:

$$
\begin{gathered}
{\left[\begin{array}{c}
\mathbf{y}_{k+N_{f}-1} \\
\mathbf{y}_{k+N_{f}-2} \\
\vdots \\
\mathbf{y}_{k}
\end{array}\right]=\left[\begin{array}{ccccccc}
\mathbf{h}_{0} & \mathbf{h}_{1} & \cdots & \mathbf{h}_{v} & 0 & \cdots & 0 \\
0 & \mathbf{h}_{0} & \mathbf{h}_{1} & \cdots & \mathbf{h}_{v} & 0 & \cdots \\
\vdots & & & & & & \vdots \\
0 & \cdots & 0 & \mathbf{h}_{0} & \mathbf{h}_{1} & \cdots & \mathbf{h}_{v}
\end{array}\right]\left[\begin{array}{c}
\mathbf{x}_{k+N_{f}-1} \\
\mathbf{x}_{k+N_{f}-2} \\
\vdots \\
\mathbf{x}_{k-v}
\end{array}\right]+} \\
\\
{\left[\begin{array}{c}
\mathbf{n}_{k+N_{f}-1} \\
\mathbf{n}_{k+N_{f}-2} \\
\vdots \\
\mathbf{n}_{k}
\end{array}\right] .}
\end{gathered}
$$

In compact vector-matrix form:

$$
\mathbf{y}_{k+N_{f}-1: k}=\mathbf{H x}_{k+N_{f}-1: k-v}+\mathbf{n}_{k+N_{f}-1: k} .
$$

In the above equation we used the compact notation $k+N_{f}-1: k-v$ to denote a vector whose first and last components takes the subscripts $k+N_{f}-1, k-v$ respectively. We denote the FIR FFF with $\mathbf{w}^{\dagger}$ where:

$$
\begin{aligned}
\mathbf{w}^{\dagger} & =\left[\mathbf{w}_{-\left(N_{f}-1\right)}^{\dagger}, . ., \mathbf{w}_{0}^{\dagger}\right] \\
\mathbf{w}_{m}^{\dagger} & =\left[\begin{array}{lll}
w_{(N s-1), m}^{*}, & \cdots & w_{0, m}^{*}
\end{array}\right] .
\end{aligned}
$$

For convenience let us define:

$$
1_{\Delta} \triangleq\left[\begin{array}{lll}
0_{1 \times \Delta} & 1 & 0_{1 \times N_{l e}}
\end{array}\right]
$$


where $\Delta \quad\left(0 \leq \Delta \leq N_{f}+v-1\right)$ is the equalizer decision delay and $N_{l e} \triangleq N_{f}+v-\Delta-1$. At each time instant $k$, the FFF $\mathbf{w}^{\dagger}$ filters the block of output samples $\mathbf{y}_{k+N_{f}-1: k}$ to form a decision variable $z_{k}$. The error $e_{k}$ between the filtered signal $z_{k}$ and desired symbol is given by:

$$
\begin{aligned}
e_{k} & \triangleq z_{k}-x_{k+N_{f}-1-\Delta} \\
& =\sum_{l=0}^{N_{f}-1} \mathbf{w}_{-l}^{\dagger} \mathbf{y}_{k+l}-x_{k+N_{f}-1-\Delta} \\
& =\left[\begin{array}{lll}
\mathbf{w}^{\dagger}-\left(N_{f}-1\right) & \cdots & \mathbf{w}_{0}^{\dagger}
\end{array}\right] \mathbf{y}_{k+N_{f}-1: k}-\left[\begin{array}{lll}
0_{1 \times \Delta} & 1 & 0_{1 \times N_{l e}}
\end{array}\right] \mathbf{x}_{k+N_{f}-1: k-v} \\
& =\mathbf{w}^{\dagger} \mathbf{y}_{k+N_{f}-1: k}-1_{\Delta} \mathbf{x}_{k+N_{f}-1: k-v} .
\end{aligned}
$$

The optimum filter settings that minimize the MSE metric $E\left[\left|e_{k}\right|^{2}\right]$ can be determined as $[39]$

$$
\begin{aligned}
\mathbf{w}^{\dagger} & =1_{\Delta} \mathbf{R}_{x y} \mathbf{R}_{y y}^{-1} \\
& =1_{\Delta} \mathbf{R}_{x x} \mathbf{H}^{\dagger}\left[\mathbf{H} \mathbf{R}_{x x} \mathbf{H}^{\dagger}+\mathbf{R}_{n n}\right]^{-1} \\
& =1_{\Delta}\left[\mathbf{R}_{x x}^{-1}+\mathbf{H}^{\dagger} \mathbf{R}_{n n}^{-1} \mathbf{H}\right]^{-1} \mathbf{H}^{\dagger} \mathbf{R}_{n n}^{-1}
\end{aligned}
$$

where

$$
\begin{aligned}
& \mathbf{R}_{x x} \triangleq E\left[\mathbf{x}_{k+N_{f}-1: k-v} \mathbf{x}_{k+N_{f}-1: k-v}^{\dagger}\right]=\mathbf{I} \\
& \mathbf{R}_{n n} \triangleq E\left[\mathbf{n}_{k+N_{f}-1: k} \mathbf{n}_{k+N_{f}-1: k}^{\dagger}\right]=s N_{0} \mathbf{I} \\
& \mathbf{R}_{x y} \triangleq E\left[\mathbf{x}_{k+N_{f}-1: k-v} \mathbf{y}_{k+N_{f}-1: k}^{\dagger}\right]=\mathbf{R}_{x x} \mathbf{H}^{\dagger} \\
& \mathbf{R}_{y y} \triangleq E\left[\mathbf{y}_{k+N_{f}-1: k} \mathbf{y}_{k+N_{f}-1: k}^{\dagger}\right]=\mathbf{H R}_{x x} \mathbf{H}^{\dagger}+\mathbf{R}_{n n} .
\end{aligned}
$$

The minimum MSE is given by:

$$
\mathrm{MSE}_{\text {Conv FIR LE }}=1_{\Delta}\left[\mathbf{R}_{x x}^{-1}+\mathbf{H}^{\dagger} \mathbf{R}_{n n}^{-1} \mathbf{H}\right]^{-1} 1_{\Delta}^{\dagger} .
$$

The optimum filter settings are determined by the decision-delay value $\Delta$ that corresponds to the minimum MSE. 


\subsubsection{Finite Length WL Linear Equalizer}

To construct a finite length WL-LE we begin with the I-Q signal model (2.6)

$$
\begin{aligned}
\tilde{\mathbf{y}}_{k} & =\tilde{\mathbf{h}}_{k} \circledast x_{k}+\tilde{\mathbf{n}}_{k} \\
& =\sum_{m=0}^{v} \tilde{\mathbf{h}}_{m} x_{k-m}+\tilde{\mathbf{n}}_{k} .
\end{aligned}
$$

Collecting a block of $N_{f}$ samples of $\tilde{\mathbf{y}}_{k}$ in vector-matrix format:

$$
\begin{gathered}
{\left[\begin{array}{c}
\tilde{\mathbf{y}}_{k+N_{f}-1} \\
\tilde{\mathbf{y}}_{k+N_{f}-2} \\
\vdots \\
\tilde{\mathbf{y}}_{k}
\end{array}\right]=\left[\begin{array}{ccccccc}
\tilde{\mathbf{h}}_{0} & \tilde{\mathbf{h}}_{1} & \cdots & \tilde{\mathbf{h}}_{v} & 0 & \cdots & 0 \\
0 & \tilde{\mathbf{h}}_{0} & \tilde{\mathbf{h}}_{1} & \cdots & \tilde{\mathbf{h}}_{v} & 0 & \cdots \\
\vdots & & & & & & \vdots \\
0 & \cdots & 0 & \tilde{\mathbf{h}}_{0} & \tilde{\mathbf{h}}_{1} & \cdots & \tilde{\mathbf{h}}_{v}
\end{array}\right]\left[\begin{array}{c}
\mathbf{x}_{k+N_{f}-1} \\
\mathbf{x}_{k+N_{f}-2} \\
\vdots \\
\mathbf{x}_{k-v}
\end{array}\right]+} \\
\\
{\left[\begin{array}{c}
\tilde{\mathbf{n}}_{k+N_{f}-1} \\
\tilde{\mathbf{n}}_{k+N_{f}-2} \\
\vdots \\
\tilde{\mathbf{n}}_{k}
\end{array}\right] .}
\end{gathered}
$$

In compact vector-matrix form:

$$
\tilde{\mathbf{y}}_{k+N_{f}-1: k}=\tilde{\mathbf{H}} \mathbf{x}_{k+N_{f}-1: k-v}+\tilde{\mathbf{n}}_{k+N_{f}-1: k} .
$$

Since each element in $\tilde{\mathbf{y}}_{k+N_{f}-1: k}, \tilde{\mathbf{n}}_{k+N_{f}-1: k}, \tilde{\mathbf{H}}$ is made up of a $2 s N \times 1$ column vectors composed of in-phase and quadrature components, the actual size of these quantities are $2 N s N_{f} \times 1,2 N s N_{f} \times 1,2 N s N_{f} \times\left(N_{f}+v\right)$ respectively. In this case, we independently filter the I/Q data branches to produce a decision variable

$$
\tilde{z}_{k}=\tilde{\mathbf{w}}^{\dagger} \tilde{\mathbf{y}}_{k+N_{f}-1: k}
$$

where $\tilde{\mathbf{w}}^{\dagger}$ denotes the WL FFF. The error $\tilde{e}_{k}$ between the filtered signal and desired symbol is given by:

$$
\tilde{e}_{k} \triangleq \tilde{z}_{k}-x_{k+N_{f}-1-\Delta}=\tilde{\mathbf{w}}^{\dagger} \tilde{\mathbf{y}}_{k+N_{f}-1: k}^{\dagger}-1_{\Delta} \mathbf{x}_{k+N_{f}-1: k-v} .
$$


The optimum filter settings that minimize the MSE metric $E\left[\left|\tilde{e}_{k}\right|^{2}\right]$ can be determined as:

$$
\begin{aligned}
\tilde{\mathbf{w}}^{\dagger} & =1_{\Delta} \mathbf{R}_{x \tilde{y}} \mathbf{R}_{\tilde{y} \tilde{y}} \\
& =1_{\Delta} \mathbf{R}_{x x} \tilde{\mathbf{H}}^{\dagger}\left[\tilde{\mathbf{H}} \mathbf{R}_{x x} \tilde{\mathbf{H}}^{\dagger}+\mathbf{R}_{\tilde{n} \tilde{n}}\right]^{-1} \\
& =1_{\Delta}\left[\mathbf{R}_{x x}^{-1}+\tilde{\mathbf{H}}^{\dagger} \mathbf{R}_{\tilde{n} \tilde{n}} \tilde{\mathbf{H}}\right]^{-1} \tilde{\mathbf{H}}^{\dagger} \mathbf{R}_{\tilde{n} \tilde{n}}^{-1}
\end{aligned}
$$

where

$$
\begin{aligned}
& \mathbf{R}_{\tilde{n} \tilde{n}} \triangleq E\left[\tilde{\mathbf{n}}_{k+N_{f}-1: k} \tilde{\mathbf{n}}_{k+N_{f}-1: k}^{\dagger}\right]=\frac{s N_{0}}{2} \mathbf{I} \\
& \mathbf{R}_{x \tilde{y}} \triangleq E\left[\mathbf{x}_{k+N_{f}-1: k-v} \tilde{\mathbf{y}}_{k+N_{f}-1: k}^{\dagger}\right]=\mathbf{R}_{x x} \tilde{\mathbf{H}}^{\dagger} \\
& \mathbf{R}_{\tilde{y} \tilde{y}} \triangleq E\left[\tilde{\mathbf{y}}_{k+N_{f}-1: k} \tilde{\mathbf{y}}_{k+N_{f}-1: k}^{\dagger}\right]=\tilde{\mathbf{H}} \mathbf{R}_{x x} \tilde{\mathbf{H}}^{\dagger}+\mathbf{R}_{\tilde{n} \tilde{n}} .
\end{aligned}
$$

The minimum MSE is given by:

$$
\mathrm{MSE}_{\text {WL FIR LE }}=1_{\Delta}\left[\mathbf{R}_{x x}^{-1}+\tilde{\mathbf{H}}^{\dagger} \mathbf{R}_{\tilde{n} \tilde{n}}^{-1} \tilde{\mathbf{H}}\right]^{-1} 1_{\Delta}^{\dagger} .
$$

If we compare the expressions for the FFF for conventional case given in (3.25) and WL case given by (3.33), we notice that conventional method involves a complex matrix inversion operation whereas WL case requires inversion of a real matrix of same dimension. Apart from this difference, as mentioned in the previous section, the WL case involves MF of real signal whereas conventional filter requires complex valued MF's. Hence, WL-LE provides a complexity advantage in a finite length implementation. Next we examine the MSE connection between the WL and conventional LE for finite length case. 


\subsubsection{MSE Comparison for Finite Length LE}

We fix the FFF filter length to be $N_{f}$ and assume the same decision delay in both cases then compare the MSE. Recall that the MSE for a conventional LE is given by (3.26) as:

$$
\mathrm{MSE}_{\text {Conv FTR LE }}=1_{\Delta}\left[\mathbf{I}+\frac{2 \mathbf{H}^{\dagger} \mathbf{H}}{N_{0}}\right]^{-1} 1_{\Delta}^{\dagger} .
$$

Let $\mathbf{I}+\frac{2 \mathbf{H}^{\dagger} \mathbf{H}}{N_{0}}=\mathbf{A}+j \mathbf{B}$. Using this we can express the MSE as:

$$
\operatorname{MSE}_{\text {Conv FIR LE }}=1_{\Delta}[\mathbf{A}+j \mathbf{B}]^{-1} 1_{\Delta}^{\dagger}=1_{\Delta}\left[\mathbf{I}+j \mathbf{A}^{-1} \mathbf{B}\right]^{-1} \mathbf{A}^{-1} 1_{\Delta}^{\dagger} .
$$

Further let $\mathbf{C}=j \mathbf{A}^{-1} \mathbf{B}$. Using an infinite series expansion:

$$
[\mathbf{I}+\mathbf{C}]^{-1}=\mathbf{I}+\sum_{m=1}^{\infty}(-1)^{m} \mathbf{C}^{m}
$$

and separating the odd and even powered terms, the MSE can be represented as an infinite series

$$
\mathrm{MSE}_{\mathrm{Conv} \text { FIR LE }}=1_{\Delta} \mathbf{A}^{-1} 1_{\Delta}^{\dagger}-\sum_{m, \text { odd }}^{\infty} 1_{\Delta} \mathbf{C}^{m} \mathbf{A}^{-1} 1_{\Delta}^{\dagger}+\sum_{m, \text { even }}^{\infty} 1_{\Delta} \mathbf{C}^{m} \mathbf{A}^{-1} 1_{\Delta}^{\dagger} .
$$

Using the special properties of $\mathbf{C}$ (see Section 3.3.6, Theorem 3.3.1) we can show that:

- The the eigenvalues of $\mathbf{C}$ are less than 1 and hence the series is absolutely convergent.

- Since the diagonal entries of $\mathbf{C}^{m} \mathbf{A}^{-1}$ are zero for odd values of $m$, the term $1_{\Delta} \mathbf{C}^{m} \mathbf{A}^{-1} 1_{\Delta}^{\dagger}$ becomes zero.

- Since $\mathbf{C}^{m} \mathbf{A}^{-1}$ is positive semi-definite for $m$ even, $1_{\Delta} \mathbf{C}^{m} \mathbf{A}^{-1} 1_{\Delta}^{\dagger} \geq 0$.

Using the above results we can express the MSE of conventional FIR LE in terms of MSE of WL FIR LE as:

$$
\begin{aligned}
\mathrm{MSE}_{\mathrm{Conv} \mathrm{FIR} \mathrm{LE}} & =1_{\Delta} \mathbf{A}^{-1} 1_{\Delta}^{\dagger}+\sum_{m, \text { even }}^{\infty} 1_{\Delta} \mathbf{C}^{m} \mathbf{A}^{-1} 1_{\Delta}^{\dagger} \\
& =\mathrm{MSE}_{\mathrm{WL} \text { FIR LE }}+\text { Some positive constant }
\end{aligned}
$$


Table 3.1. LE performance comparison in channel-1

\begin{tabular}{|c|c|c|c|c|}
\hline$N_{f}$ & $M S E_{\text {Conv }}$ & $M S E_{W L}$ & $S N R_{U, \text { Conv }}(\mathrm{dB})$ & $S N R_{U, W L}(\mathrm{~dB})$ \\
\hline 5 & 0.2142 & 0.0476 & 5.64 & 13 \\
\hline 10 & 0.1683 & 0.0476 & 6.94 & 13 \\
\hline 15 & 0.1583 & 0.0476 & 7.26 & 13 \\
\hline
\end{tabular}

where we used the MSE expression given by (3.34): $\operatorname{MSE}_{\mathrm{WL} \text { FIR LE }}=1_{\Delta} \mathbf{A}^{-1} 1_{\Delta}^{\dagger}$. The above result suggests that, for any given filter length $N_{f}$, and any given decision delay, the conventional FIR LE exhibits a higher MSE than WL FIR LE for all complex valued ISI channels. Equality holds only when the imaginary part of the signal covariance is zero i.e. $\mathbf{B}=0$. Hence we can say that while the $\mathrm{WL}$ receiver optimally exploits the signal energy for detection, the conventional LE suffers from additional noise enhancement because of the presence of imaginary component $\mathbf{B}$ in the signal covariance. The noise enhancement depends mainly the diagonal entries of $\mathbf{A}^{-1} \mathbf{B}^{m} \mathbf{A}^{-1}$. To understand the trade-off between MSE and complexity we revisit Examples-1 and 2 for the finite length case.

Table 3.1 shows the performance of channel-1 given in Example 1 and in Table 3.2 we show the results for channel-2 given in Example 2. In the second case, the conventional LE provides near infinite-length performance for a 10 tap filter whereas WL-LE nearly achieves its infinite length SNR with 5 taps only; a great deal of complexity reduction. On the other hand, if we fix the filter length to 5 taps, then WL-LE provides $2.5 \mathrm{~dB}$ SNR gain over conventional LE while we recall that the SNR difference between the two methods is only $1.5 \mathrm{~dB}$ when both methods use infinite length filters. Clearly, WLLE significantly outperforms conventional LE both in complexity and performance. 
Table 3.2. LE performance comparison in channel-2

\begin{tabular}{|c|c|c|c|c|}
\hline$N_{f}$ & $M S E_{\text {Conv }}$ & $M S E_{W L}$ & $S N R_{U, C o n v}(\mathrm{~dB})$ & $S N R_{U, W L}(\mathrm{~dB})$ \\
\hline 5 & 0.1793 & 0.1095 & 6.6 & 9.1 \\
\hline 10 & 0.1465 & 0.1081 & 7.65 & 9.16 \\
\hline 15 & 0.1462 & 0.1081 & 7.66 & 9.16 \\
\hline
\end{tabular}

\subsection{MMSE Decision Feedback Equalization}

In case of LE we have shown that WL-LE offers lower noise enhancement, lower MSE and thus outperforms conventional LE in complex valued ISI channels. In this section we consider the performance of conventional and WL-DFE receivers. We first analyze the infinite length DFE followed by finite length DFE.

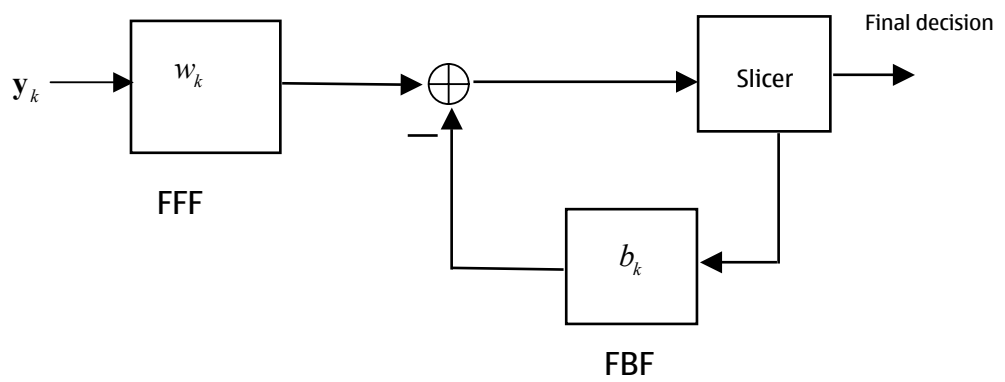

Figure 3.3. Conventional MMSE-DFE.

\subsubsection{Infinite Length Conventional DFE}

We define the DFE settings for the conventional signal model given by (2.5)

$$
\mathbf{y}_{k}=\mathbf{h}_{k} \circledast x_{k}+\mathbf{n}_{k} .
$$

A standard MMSE-DFE structure consists of (see Figure 3.3) a vector-valued feedforward filter $(\mathrm{FFF}) \mathbf{w}_{k}=\left[w_{1, k}, . ., w_{s N, k}\right]$, that filters the input data (column) vector $\mathbf{y}_{k}$ such that at the output a symbol-by-symbol detector makes decisions by removing 
ISI (using past symbol estimates) with the aid of a feedback filter (FBF) $b_{k}$. The filters are optimized by minimizing the MSE term: $\left[\mathbf{w}_{k}, b_{k}\right]=\min _{\left[\mathbf{w}_{k}, b_{k}\right]} E\left[\left|e_{k}\right|^{2}\right]$ where $e_{k}$ is the error between filtered signal and the reconstructed signal defined as:

$$
e_{k}=\mathbf{w}_{k} \circledast \mathbf{y}_{k}-b_{k} \circledast x_{k}
$$

Here $b_{k} \circledast x_{k}$ represents the reconstructed signal term where we have assumed perfect knowledge of past symbol estimates. The feedback filter is constrained to be causal, monic i.e.

$$
\begin{aligned}
b_{k} & =1, \quad k=0 \\
& =0, \quad k<0 .
\end{aligned}
$$

In D-domain (3.36) can be expressed as:

$$
e(D)=\mathbf{w}(D) \mathbf{y}(D)-b(D) x(D)
$$

Using orthogonality principle [5]:

$$
E\left[e(D) \mathbf{y}^{\dagger}\left(D^{-*}\right)\right]=0 .
$$

Using (3.36), expanding the terms in (3.38), we get:

$$
\begin{aligned}
\mathbf{w}(D) & =b(D) \mathbf{R}_{\mathbf{x y}}(D) \mathbf{R}_{\mathbf{y y}}^{-\mathbf{1}}(D) \\
& =b(D) R_{x x}(D) \mathbf{h}^{\dagger}\left(D^{-*}\right)\left[\mathbf{h}(D) R_{x x}(D) \mathbf{h}^{\dagger}\left(D^{-*}\right)+\mathbf{R}_{\mathbf{n n}}(D)\right]^{-1} \\
& =b(D)\left[R_{x x}^{-1}(D)+\mathbf{h}^{\dagger}\left(D^{-*}\right) \mathbf{R}_{\mathbf{n n}}^{-\mathbf{1}}(D) \mathbf{h}(D)\right]^{-1} \mathbf{h}^{\dagger}\left(D^{-*}\right) \mathbf{R}_{\mathbf{n n}}^{-\mathbf{1}}(D)
\end{aligned}
$$

where

$$
\begin{aligned}
& \mathbf{R}_{\mathbf{x y}}(D) \triangleq R_{x x}(D) \mathbf{h}^{\dagger}\left(D^{-*}\right) \\
& \mathbf{R}_{\mathbf{y y}}(D) \triangleq\left[\mathbf{h}(D) R_{x x}(D) \mathbf{h}^{\dagger}\left(D^{-*}\right)+\mathbf{R}_{\mathbf{n n}}(D)\right] \\
& \mathbf{R}_{\mathbf{n n}}(D) \triangleq E\left[\mathbf{n}(D) \mathbf{n}^{\dagger}\left(D^{-*}\right)\right]
\end{aligned}
$$


Let us consider the scalar spectrum factorization [5]:

$$
\left[R_{x x}^{-1}(D)+\mathbf{h}^{\dagger}\left(D^{-*}\right) \mathbf{R}_{\mathbf{n n}}^{-\mathbf{1}}(D) \mathbf{h}(D)\right] \leftrightarrow \gamma_{\mathrm{Conv}} u^{\dagger}\left(D^{-*}\right) u(D)
$$

where

$$
\gamma_{\text {Conv }}=e^{T \int_{-\frac{1}{2 T}}^{\frac{1}{2 T}} \ln \left[R_{x x}^{-1}(f)+\mathbf{h}^{\dagger}(-f) \mathbf{R}_{\mathbf{n n}}^{-1}(f) \mathbf{h}(f)\right] d f}
$$

is a positive constant and $u(D)$ is a causal, monic, minimum phase function with all poles and zeros inside the unit circle, and $u^{\dagger}\left(D^{-*}\right)$ is anti-causal, maximum phase function. It can be shown that the optimum FBF that minimizes the MSE is given by:

$$
b(D)=u(D)
$$

Using this, the optimum FFF can be expressed in terms of spectral factors as

$$
\begin{aligned}
\mathbf{w}(D) & =b(D)\left[R_{x x}^{-1}(D)+\mathbf{h}^{\dagger}\left(D^{-*}\right) \mathbf{R}_{\mathbf{n n}}^{-\mathbf{1}}(D) \mathbf{h}(D)\right]^{-1} \mathbf{h}^{\dagger}\left(D^{-*}\right) \mathbf{R}_{\mathbf{n n}}^{-\mathbf{1}}(D) \\
& =\left[\gamma_{\text {conv }}\right]^{-1} u^{\dagger}\left(D^{-*}\right)^{-1} \mathbf{h}^{\dagger}\left(D^{-*}\right) \mathbf{R}_{\mathbf{n n}}^{-\mathbf{1}}(D)
\end{aligned}
$$

In Appendix B.1 we described an algorithm that can be used to calculate the spectral factors $u(D)$ and the inverse filter $u^{\dagger}\left(D^{-*}\right)^{-1}$ that can be used to determine the FFF settings. The minimum MSE for this method is given by:

$$
\mathrm{MSE}_{\text {Conv DFE }}=\frac{1}{\gamma_{\text {Conv }}}=e^{-T \int_{-\frac{1}{2 T}}^{\frac{1}{2 T}} \ln \left[R_{x x}^{-1}(f)+\mathbf{h}^{\dagger}(-f) \mathbf{R}_{\mathbf{n n}}^{-1}(f) \mathbf{h}(f)\right] d f} .
$$

Although the MMSE-DFE structure is specifically designed for a DFE symbol detector, the FFF is quite often used as a pre-filter for RSSE [9] or MLSE detectors.

\subsubsection{Infinite Length WL DFE}

Unlike conventional MMSE-DFE which works with complex valued received signal (2.5), we define DFE settings for the I-Q signal model (2.6):

$$
\tilde{\mathbf{y}}_{k}=\tilde{\mathbf{h}}_{k} \circledast x_{k}+\tilde{\mathbf{n}}_{k} .
$$


The structural form of WL-DFE is shown in Figure 3.4. Let:

$$
\tilde{\mathbf{e}}_{k}=\tilde{\mathbf{w}}_{k} \circledast \tilde{\mathbf{y}}_{k}-\tilde{b}_{k} \circledast x_{k}
$$

be the error between the pre-filtered signal and the re-constructed signal, and

$$
\tilde{\mathbf{w}}_{k}=\left[\mathbf{w}_{I, k}, \mathbf{w}_{Q, k}\right]
$$

denotes the WL-FFF of dimension $1 \times 2 s N$ and $\tilde{b}_{k}$ is a real-valued scalar feedback filter. The feedback filter is again assumed to be causal, monic. Using the results from the previous section, we can obtain the WL-DFE filter settings as:

$$
\begin{aligned}
\tilde{\mathbf{w}}(D) & =\tilde{b}(D)\left[R_{x x}^{-1}(D)+\tilde{\mathbf{h}}^{\dagger}\left(D^{-*}\right) \mathbf{R}_{\tilde{\mathbf{n}} \tilde{\mathbf{n}}}^{-\mathbf{1}}(D) \tilde{\mathbf{h}}(D)\right]^{-1} \tilde{\mathbf{h}}^{\dagger}\left(D^{-*}\right) \mathbf{R}_{\tilde{\mathbf{n}} \tilde{\mathbf{n}}}^{-\mathbf{1}}(D) \\
& =\left[\gamma_{\mathrm{wL}}\right]^{-1} \tilde{u}^{\dagger}\left(D^{-*}\right)^{-1} \tilde{\mathbf{h}}^{\dagger}\left(D^{-*}\right) \mathbf{R}_{\tilde{\mathbf{n}} \tilde{\mathbf{n}}}^{-\mathbf{1}}(D) \\
\tilde{b}(D) & =\tilde{u}(D)
\end{aligned}
$$

where $\tilde{u}(D)$ is obtained by factoring the real valued covariance function as:

$$
\left[R_{x x}^{-1}(D)+\tilde{\mathbf{h}}^{\dagger}\left(D^{-*}\right) \mathbf{R}_{\tilde{\mathbf{n}} \tilde{\mathbf{n}}}^{-\mathbf{1}}(D) \tilde{\mathbf{h}}(D)\right] \leftrightarrow \gamma_{\mathrm{wL}} \tilde{u}^{\dagger}\left(D^{-*}\right) \tilde{u}(D)
$$

where

$$
\gamma_{\mathrm{WL}}=e^{T \int_{-\frac{1}{2 T}}^{\frac{1}{2 T}} \ln \left[R_{x x}^{-1}(f)+\tilde{\mathbf{h}}^{\dagger}(-f) \mathbf{R}_{\tilde{\mathbf{n}} \tilde{\mathbf{n}}}^{-1}(f) \tilde{\mathbf{h}}(f)\right] d f}
$$

is a positive constant and $\tilde{u}(D)$ is a causal, monic, minimum phase function with all poles and zeros inside the unit circle, and $\tilde{u}^{\dagger}\left(D^{-*}\right)$ is anti causal, maximum phase function. In case of white noise, we can represent the WL-FFF alternatively as

$$
\begin{aligned}
& \tilde{\mathbf{w}}(D)=\left[\gamma_{\mathrm{wL}} \frac{s N_{0}}{2}\right]^{-1} \tilde{u}^{\dagger}\left(D^{-*}\right)^{-1} \tilde{\mathbf{h}}^{\dagger}\left(D^{-*}\right) \\
& =\left[\gamma_{\mathrm{WL}} \frac{s N_{0}}{2}\right]^{-1} \tilde{u}^{\dagger}\left(D^{-*}\right)^{-1}\left[\mathbf{h}_{I}^{\dagger}\left(D^{-*}\right) \quad \mathbf{h}_{Q}^{\dagger}\left(D^{-*}\right)\right] \\
& =\left[\begin{array}{ll}
\mathbf{w}_{I}(D) & \mathbf{w}_{Q}(D)
\end{array}\right] \text {. }
\end{aligned}
$$


The minimum MSE for these settings is given by:

$$
\operatorname{MSE}_{\mathrm{WL}} \text { DFE }=\frac{1}{\gamma_{\mathrm{WL}}}=e^{-T \int_{-\frac{1}{2 T}}^{\frac{1}{2 T}} \ln \left[R_{x x}^{-1}(f)+\tilde{\mathbf{h}}^{\dagger}(-f) \mathbf{R}_{\tilde{\mathbf{n}} \tilde{\mathbf{n}}}^{-1}(f) \tilde{\mathbf{h}}(f)\right] d f} .
$$

As in conventional case, DFE, RSSE/MLSE methods can be straightforwardly used after WL-FFF.

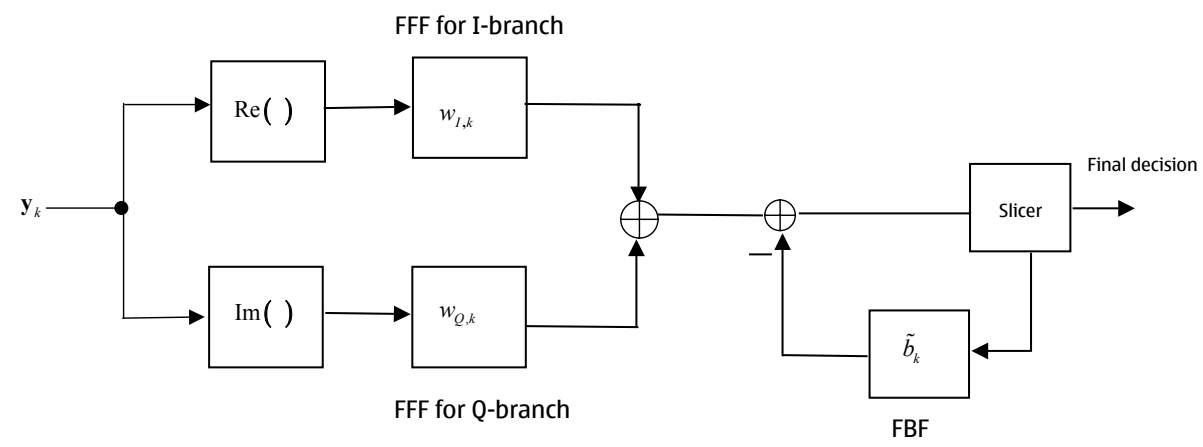

Figure 3.4. WL MMSE-DFE.

\subsubsection{MSE Comparison for Infinite Length DFE}

In Appendix A.1 we show that the MSE at the output of a conventional MMSE-DFE is always higher than or equal to the MSE produced by WL MMSE-DFE. The two MSE metrics are related as:

$$
\operatorname{MSE}_{\text {Conv DFE }}=\text { MSE }_{\text {WL DFE }} e^{T \int_{-\frac{1}{2 T}}^{\frac{1}{2 T}}\left[\frac{\bar{a}^{2}(f)}{2 a^{2}(f)}+\frac{\bar{a}^{4}(f)}{4 a^{4}(f)}+. .\right] d f} .
$$

In the above expression the multiplicative factor $e^{T \int_{-\frac{1}{2 T}}^{\frac{1}{2 T}}\left[\frac{\bar{a}^{2}(f)}{2 a^{2}(f)}+\frac{\bar{a}^{4}(f)}{4 a^{4}(f)}+. .\right] d f}$ is always greater than or equal to 1 in any complex-valued ISI channel. Equality occurs only when $\bar{a}(f)$ becomes zero.

Next we revisit Examples 1 and 2 and evaluate the MSE for these channels with infinite length DFE filters. For channel-1, using numerical integration we get:

$$
\mathrm{MSE}_{\text {Conv DFE }} \sim 0.07
$$


at $\mathrm{SNR}=10 \mathrm{~dB}$. The unbiased $\mathrm{SNR}$ is $\mathrm{SNR}_{\mathrm{U}, \text { Conv } \mathrm{DFE}}=11.23 \mathrm{~dB}$ which is $1.77 \mathrm{~dB}$ below MFB. Note that the conventional DFE itself provides remarkable gain over conventional LE (which suffers from severe noise enhancement) whereas both WL-LE and WL-DFE are able to achieve optimum MFB in this case.

In channel-2 we get: $\mathrm{MSE}_{\mathrm{Conv} \text { DFE }} \sim 0.1$ at $\mathrm{SNR}=10 \mathrm{~dB}$. The unbiased $\mathrm{SNR}$ is $\mathrm{SNR}_{\mathrm{U}, \mathrm{Conv} \text { DFE }}=$ $9.54 \mathrm{~dB}$ which is $1.97 \mathrm{~dB}$ better than conventional LE and $3.54 \mathrm{~dB}$ below MFB. In

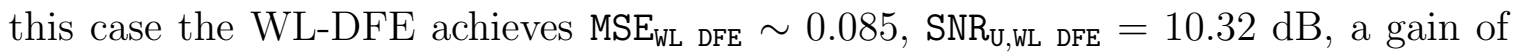
$0.78 \mathrm{~dB}$ gain over conventional DFE.

\subsubsection{Conventional Finite Length MMSE-DFE}

Infinite length DFE analysis is only a limiting situation and we wish to understand the performance, complexity trade-offs between conventional and WL-DFE receivers with finite length constraints. To this end we first analyze the conventional FIR DFE followed by WL case. Recall that the conventional signal model in block matrix form is given by $(3.22)$

$$
\mathbf{y}_{k+N_{f}-1: k}=\mathbf{H x}_{k+N_{f}-1: k-v}+\mathbf{n}_{k+N_{f}-1: k} .
$$

As in FIR conventional LE we denote the FFF with $\mathbf{w}^{\dagger}$ where:

$$
\begin{aligned}
\mathbf{w}^{\dagger} & =\left[\mathbf{w}_{-\left(N_{f}-1\right)}^{\dagger}, . ., \mathbf{w}_{0}^{\dagger}\right] \\
\mathbf{w}_{m}^{\dagger} & =\left[\begin{array}{lll}
w_{(N s-1), m}^{*}, & \cdots & w_{0, m}^{*}
\end{array}\right] .
\end{aligned}
$$

In case of DFE, the FFF $\mathbf{w}^{\dagger}$ filters the block of output samples $\mathbf{y}_{k+N_{f}-1: k}$ and a feedback filter $\mathbf{b}^{\dagger}$ removes ISI with the aid of $N_{b}$ past decisions. For convenience let us define:

$$
\mathbf{b}^{\dagger} \triangleq\left[\begin{array}{llllll}
0_{1 \times \Delta} & 1 & b^{*}{ }_{1} & \cdots & b_{N_{b}}^{*} & 0_{1 \times N_{e}}
\end{array}\right]
$$


where $b^{*}{ }_{l}$ denotes the FBF coefficients. The error $e_{k}$ between the filtered signal and desired symbol is given by

$$
\begin{aligned}
e_{k} & \triangleq z_{k}-x_{k+N_{f}-1-\Delta} \\
& =\sum_{l=0}^{N_{f}-1} \mathbf{w}_{-l}^{\dagger} \mathbf{y}_{k+l}-\sum_{m=1}^{N_{b}} b_{m}^{*} x_{k+N_{f}-1-\Delta-m}-x_{k+N_{f}-1-\Delta} \\
& =\left[\begin{array}{lll}
\mathbf{w}^{\dagger}-\left(N_{f}-1\right) & \cdots & \mathbf{w}_{0}^{\dagger}
\end{array}\right] \mathbf{y}_{k+N_{f}-1: k}^{\dagger}-\mathbf{b}^{\dagger} \mathbf{x}_{k+N_{f}-1: k-v} \\
& =\mathbf{w}^{\dagger} \mathbf{y}_{k+N_{f}-1: k}^{\dagger}-\mathbf{b}^{\dagger} \mathbf{x}_{k+N_{f}-1: k-v}
\end{aligned}
$$

where $z_{k}$ denotes the filtered received signal, $\Delta \quad\left(0 \leq \Delta N_{f}+v-1\right)$ is the decision delay and $N_{e} \triangleq N_{f}+v-\Delta-N_{b}-1$. The filter settings are obtained by minimizing the MSE [39]:

$$
\mathrm{MSE}_{\text {Conv FIR DFE }}=\mathbf{b}^{\dagger}\left[\mathbf{R}_{x x}^{-1}+\mathbf{H}^{\dagger} \mathbf{R}_{n n}^{-1} \mathbf{H}\right]^{-1} \mathbf{b}
$$

Assuming that feed-back filter length $N_{b}$ is equal to the channel memory $v$, we can obtain the optimum feedback filter setting using the matrix Cholesky factorization:

$$
\mathbf{R}_{x x}^{-1}+\mathbf{H}^{\dagger} \mathbf{R}_{n n}^{-1} \mathbf{H}=\mathbf{L D L}^{\dagger}
$$

where $\mathbf{L}$ is lower triangular monic matrix and $\mathbf{D}$ is a diagonal matrix

$$
\mathbf{D} \triangleq \operatorname{diag}\left(d_{0}, d_{1}, \cdots, d_{N_{f}+v-1}\right)
$$

Let $d_{\Delta_{\mathrm{opt}}}=\max \left(d_{N_{f}-1}, d_{N_{f}}, \cdots, d_{N_{f}+v-1}\right)$ where $\Delta_{\mathrm{opt}}$ is the optimum delay value that minimizes the MSE. Then the optimum feed-back filter is given by $\mathbf{b}_{\mathrm{opt}}^{\dagger}=\mathbf{L e}_{\Delta_{\mathrm{opt}}+1}$ where $\mathbf{e}_{\Delta_{\mathrm{opt}+1}}$ denotes a unit vector with a one in the $\Delta_{\mathrm{opt}}+1$ position and zeros else where. Then minimum MSE becomes:

$$
\mathrm{MSE}_{\mathrm{Conv} \text { FIR DFE }}=d_{\Delta_{\mathrm{opt}}^{-1}}
$$


The optimum FFF can be obtained as [39]:

$$
\begin{aligned}
\mathbf{w}^{\dagger} & =\mathbf{b}_{\mathrm{opt}}^{\dagger} \mathbf{R}_{x y} \mathbf{R}_{y y}^{-1} \\
& =d_{\Delta_{\mathrm{opt}}}^{-1} \mathbf{e}_{\Delta_{\mathrm{opt}}+1} \mathbf{L}^{-1} \mathbf{H}^{\dagger} \mathbf{R}_{n n}^{-1}
\end{aligned}
$$

where $\mathbf{R}_{x y}, \mathbf{R}_{y y}, \mathbf{R}_{n n}$ are already defined in Section 3.2.5. To calculate the filter settings the spectrum factorization algorithms given in [40] can be used.

\subsubsection{WL Finite Length MMSE-DFE}

Let us begin with the I-Q signal model (3.30):

$$
\tilde{\mathbf{y}}_{k+N_{f}-1: k}=\tilde{\mathbf{H}} \mathbf{x}_{k+N_{f}-1: k-v}+\tilde{\mathbf{n}}_{k+N_{f}-1: k} .
$$

In this case the WL-FFF filter defined as

$$
\tilde{\mathbf{w}}^{\dagger}=\left[\begin{array}{lll}
\tilde{\mathbf{w}}_{-\left(N_{f}-1\right)}^{\dagger} & \cdots & \tilde{\mathbf{w}}_{0}^{\dagger}
\end{array}\right], \quad \tilde{\mathbf{w}}_{m}^{\dagger}=\left[\begin{array}{lll}
\tilde{w}_{(2 N s-1), m} & \cdots & \tilde{w}_{0, m}
\end{array}\right]
$$

where each element $\tilde{w}_{m}$ is composed of $2 s N$ real filter coefficients. Let define a realvalued WL feed-back filter:

$$
\tilde{\mathbf{b}}^{\dagger} \triangleq\left[\begin{array}{llllll}
0_{1 \times \tilde{\Delta}} & 1 & \tilde{b}_{1} & \cdots & \tilde{b}_{N_{b}} & 0_{1 \times N_{e}}
\end{array}\right]
$$

where $N_{e} \triangleq N_{f}+v-\tilde{\Delta}-N_{b}-1$. Using these definitions we can represent the error signal as:

$$
\tilde{e}_{k} \triangleq \tilde{z}_{k}-x_{k+N_{f}-1-\tilde{\Delta}}=\tilde{\mathbf{w}}^{\dagger} \tilde{\mathbf{y}}_{k+N_{f}-1: k}^{\dagger}-\tilde{\mathbf{b}}^{\dagger} \mathbf{x}_{k+N_{f}-1: k-v}
$$

where $\tilde{z}_{k}$ denotes the filtered received signal, $\tilde{\Delta} \quad\left(0 \leq \tilde{\Delta} \leq N_{f}+v-1\right)$ the decision delay. Assuming that feed-back filter length $N_{b}$ is equal to the channel memory $v$, we can obtain the optimum feed-back filter setting using the matrix Cholesky factorization:

$$
\mathbf{R}_{x x}^{-1}+\tilde{\mathbf{H}}^{\dagger} \mathbf{R}_{\tilde{n} \tilde{n}}^{-1} \tilde{\mathbf{H}}=\tilde{\mathbf{L}} \tilde{\mathbf{D}} \tilde{\mathbf{L}}^{\dagger}
$$


where $\tilde{\mathbf{L}}$ is lower triangular monic matrix and $\tilde{\mathbf{D}}$ is a diagonal matrix:

$$
\tilde{\mathbf{D}} \triangleq \operatorname{diag}\left(\tilde{d}_{0}, \tilde{d}_{1}, \cdots, \tilde{d}_{N_{f}+v-1}\right)
$$

Let $d_{\tilde{\Delta}_{\mathrm{opt}}}=\max \left(\tilde{d}_{N_{f}-1}, \tilde{d}_{N_{f}}, \cdots, \tilde{d}_{N_{f}+v-1}\right)$ where $\tilde{\Delta}_{\mathrm{opt}}$ is the optimum delay that minimizes the MSE. Then the optimum feed-back filter is given by: $\tilde{\mathbf{b}}_{\mathrm{opt}}^{\dagger}=\tilde{\mathbf{L}}_{\tilde{\Delta}_{\mathrm{opt}}+1}$. Then then minimum MSE becomes: MSE ${ }_{\text {wL FIR DFE }}=\tilde{d}_{\tilde{\Delta}_{\mathrm{opt}}}^{-1}$. The optimum FFF is given by [39]

$$
\begin{aligned}
\tilde{\mathbf{w}}^{\dagger} & =\tilde{\mathbf{b}}_{\mathrm{opt}}^{\dagger} \mathbf{R}_{x \tilde{y}} \mathbf{R}_{\tilde{y} \tilde{y}}^{-1} \\
& =\tilde{d}_{\tilde{\Delta}_{\mathrm{opt}}}^{-1} \mathbf{e} \dagger_{\tilde{\Delta}_{\mathrm{opt}}+1} \tilde{\mathbf{L}}^{-1} \tilde{\mathbf{H}}^{\dagger} \mathbf{R}_{\tilde{n} \tilde{n}}^{-1}
\end{aligned}
$$

where $\mathbf{R}_{x \tilde{y}}, \mathbf{R}_{\tilde{y} \tilde{y}}, \mathbf{R}_{\tilde{n} \tilde{n}}$ are already introduced in Section 3.2.6.

\subsubsection{MSE Comparison for Finite Length DFE}

In the infinite length case we showed that MSE for conventional DFE is always higher than WL MSE for all complex valued ISI channels. This section examines the MSE connection in FIR case. Using (3.49), let us denote:

$$
\mathbf{Q}=\mathbf{R}_{x x}^{-1}+\mathbf{H}^{\dagger} \mathbf{R}_{n n}^{-1} \mathbf{H}
$$

As in infinite length case we use the modified noise variance term $\mathbf{R}_{n n}=\frac{N_{0}}{2} \mathbf{I}$ to facilitate a direct comparison with WL-DFE. Assuming $\mathbf{R}_{x x}=\mathbf{I}$, let us re-write $\mathbf{Q}$ in terms of real and imaginary parts as:

$$
\mathbf{Q}=\mathbf{A}+j \mathbf{B}=\mathbf{A}\left[\mathbf{I}+j \mathbf{A}^{-1} \mathbf{B}\right]
$$

where $\mathbf{A}=\mathbf{I}+\frac{2 \operatorname{Re}\left[\mathbf{H}^{\dagger} \mathbf{H}\right]}{N_{0}}$ and $\mathbf{B}=\frac{2 \operatorname{Im}\left[\mathbf{H}^{\dagger} \mathbf{H}\right]}{N_{0}}$. Now we have the following:

Theorem 3.3.1 • A is equal to the WL covariance term $\mathbf{A}=\mathbf{I}+\frac{2 \tilde{\mathbf{H}}^{\dagger} \tilde{\mathbf{H}}}{N_{0}}$ which is positive definite by definition 
- $\mathbf{B}$ is a skew Hermitian matrix whose eigenvalues are purely imaginary and appear as conjugate pairs.

- Every eigenvalue of $j \mathbf{A}^{-1} \mathbf{B}$ lies in the interval $(-1,1)$ and occur in negated pairs as $(-\lambda, \lambda)$

- $0<\left|\mathbf{I}+j \mathbf{A}^{-1} \mathbf{B}\right| \leq 1$

- Let $\mathbf{C}=j \mathbf{A}^{-1} \mathbf{B}$. Then $\mathbf{C}^{m} \mathbf{A}^{-1}$ is a positive semi-definite and has non-negative eigenvalues for $m$ even, and it becomes a skew-Hermitian matrix with zero diagonal for odd values of $m$

Proof The proof is given in Appendix A.2

Next, we examine the MSE connection between conventional and WL-DFE methods using a geometric SNR measure. Using the notion of geometrical signal energy for a multidimensional random process introduced in [41] ${ }^{4}$, we can define the geometric SNR for the conventional and I-Q covariance matrices as

$$
\begin{aligned}
\mathrm{GSNR}_{\text {Conv FIR DFE }} & \triangleq|\mathbf{A}+j \mathbf{B}|^{\frac{1}{N_{f}+v}}=|\mathbf{A}|^{\frac{1}{N_{f}+v}}\left|\mathbf{I}+j \mathbf{A}^{-1} \mathbf{B}\right|^{\frac{1}{N_{f}+v}} \\
\operatorname{GSNR}_{\text {WL FIR DFE }} & \triangleq|\mathbf{A}|^{\frac{1}{N_{f}+v}} .
\end{aligned}
$$

Since $\left|\mathbf{I}+j \mathbf{A}^{-1} \mathbf{B}\right| \leq 1$, the conventional GSNR is generally less than that of WL DFE receiver. As the filter lengths become infinite, the GMSE defined as: GMSE $\triangleq \frac{1}{G \text { GNR }}$, approaches the MSE for infinite length case, that is

$$
\begin{gathered}
\lim _{N_{f} \rightarrow \infty} \frac{1}{\operatorname{GSNR}_{\text {Conv FIR DFE }}} \rightarrow e^{-T \int_{-\frac{1}{2 T}}^{\frac{1}{2 T}} \ln \left[1+\frac{2|\mathbf{h}(f)|^{2}}{N_{0}}\right] d f} \\
\lim _{N_{f} \rightarrow \infty} \frac{1}{\operatorname{GSNR}_{\text {WL FIR DFE }}} \rightarrow e^{-T \int_{-\frac{1}{2 T}}^{\frac{1}{2 T}} \ln \left[1+\frac{2|\tilde{\mathbf{h}}(f)|^{2}}{N_{0}}\right] d f} .
\end{gathered}
$$

\footnotetext{
${ }^{4}$ A connection between geometrical SNR and channel mutual information was established in [41] for finite-length DFE
} 
Table 3.3. FIR DFE performance comparison in channel-1

\begin{tabular}{|c|c|c|c|c|}
\hline$N_{f}$ & $M S E_{\text {Conv }}$ & $M S E_{W L}$ & $S N R_{U, C o n v}(\mathrm{~dB})$ & $S N R_{U, W L}(\mathrm{~dB})$ \\
\hline 5 & 0.0766 & 0.0476 & 10.81 & 13 \\
\hline 10 & 0.0736 & 0.0476 & 10.99 & 13 \\
\hline 15 & 0.0732 & 0.0476 & 11.02 & 13 \\
\hline
\end{tabular}

Table 3.4. FIR DFE performance comparison in channel-2

\begin{tabular}{|c|c|c|c|c|}
\hline$N_{f}$ & $M S E_{\text {Conv }}$ & $M S E_{W L}$ & $S N R_{U, \text { Conv }}(\mathrm{dB})$ & $S N R_{U, W L}(\mathrm{~dB})$ \\
\hline 5 & 0.1042 & 0.0875 & 9.34 & 10.18 \\
\hline 10 & 0.1034 & 0.0868 & 9.38 & 10.22 \\
\hline 15 & 0.1034 & 0.0868 & 9.38 & 10.22 \\
\hline
\end{tabular}

From exact MSE point of view, in Appendix A.3, we show that for any given decision delay, the WL DFE provides a higher SNR (or lower MSE) than conventional DFE. Equality holds if and only if $\mathbf{B}=0$.

Let us investigate the effect of finite filter length on WL and conventional DFE receivers. We tabulated the results for channels 1 and 2 in Table 3.3 and 3.4 respectively. In both cases, the MSE does not change significantly when filter length is increased from 5 to 15. If we assume 5-taps for implementation, we get an SNR gain (between WL and conventional cases) of 2.19, $0.84 \mathrm{~dB}$ for Examples-1,2 respectively. We must also note that WL DFE is still less complex to implement than conventional DFE for a given filter length.

\subsubsection{Complexity Comparison}

In order to determine the optimum filter settings each method performs a matrix Cholesky decomposition. We should first note that the WL case [see (3.51)] involves Cholesky decomposition of real symmetric matrix whereas the conventional case [see (3.49)] needs to decompose a complex Hermitian matrix of same dimension that re- 
quires a higher computational power. Also, the MF front-end for conventional DFE case involves a complex valued filter which requires $s N$ complex convolutions (equivalent to $4 s N$ real convolutions) whereas the WL case involves $2 s N$ real convolutions. Clearly, the WL method has a computational advantage over conventional MMSEDFE.

\subsection{Summary}

In this chapter we demonstrate the complexity and performance differences between WL and conventional equalizers using MMSE and MMSE-DFE configurations and for infinite and finite length equalizers. When the ISI channel response is complex, we show that WL-LE and WL-DFE equalizers outperform their conventional counterparts both in complexity and performance. In Section 1V C of [28], the authors pointed out that they could not obtain a direct MSE connection between conventional and WL methods. We show that this can be done. Specifically, we derive closed form results that provide an explicit connection between MSE performance and computational complexity between conventional and WL equalizers for both finite and infinite length filters. We would like to note that the proposed WL equalization concepts can be extended to PAM and QAM systems to enhance performance in the presence of co-channel interference. This aspect is considered in the following chapters. 


\section{CHAPTER 4}

\section{INTERFERENCE CANCELLATION}

\subsection{Introduction}

The main theme of Chapter 3 was the lower noise enhancement properties of WL receivers for PAM equalization. For complex valued ISI channels, when the noise is white, it is shown that WL PAM equalizers, both LE and DFE, provide lower noise enhancement and lower complexity than conventional MMSE methods. In this chapter we consider performance with both ISI and CCI. Assuming that interference consists of a mixture of PAM and QAM signals (as discussed in Section 2.4), we analyze performance for conventional and WL systems with PAM/QAM signaling. When the ISI channel response is complex, and when interference has PAM components, collecting the real and imaginary parts of the received signal gives two copies of signal and interference with real channel gains. In this case, an MMSE-type receiver that exploits the correlations between the I-Q signals can be used to suppress interference. This property was used in [31] and [32] to suppress CCI in PAM-type systems. In this chapter we show that the gain of WLE is not limited to PAM; We show that QAM receivers can benefit from WLF especially when interference has PAM components. This chapter has two main contributions. First, we formulate WL MMSE/MMSE-DFE receiver structures for QAM signaling with infinite length filters. Second, we explain the performance differences between conventional and WL receivers for both PAM and QAM signaling when the receiver has multiple antennas and multiple CCI. 
In Chapter 3 we already derived MMSE/MMSE-DFE receiver structures for conventional and WL receivers for PAM signaling. Although performance analysis was restricted to white noise case, the proposed receivers are applicable for interference limited systems as well. Therefore, we shall not repeat those derivation again. In Section 4.2 of this chapter, we first generalize the WL MMSE/MMSE-DFE equalization concepts for QAM systems with multiple antennas and multiple CCI. In Section 4.3, we compare the IC performance of conventional and WL receivers for both PAM and QAM signaling schemes. The implementation complexity of proposed receivers is briefly considered in Section 4.4. Finally, simulation is used to show the IC benefits of proposed receivers in Section 4.5 followed by a summary of this chapter in Section 4.6 .

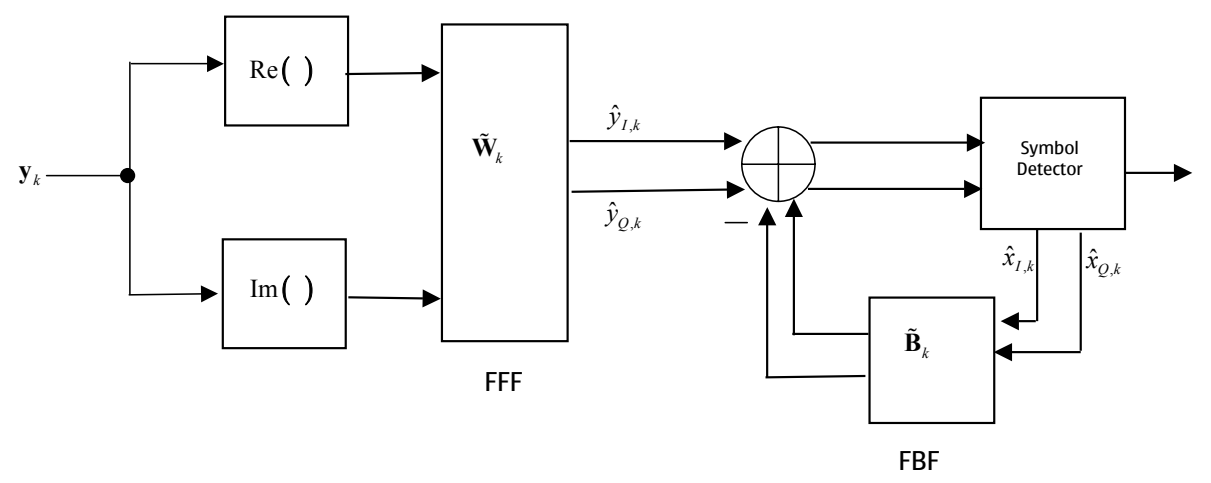

Figure 4.1. WL MMSE-DFE for QAM.

\subsection{WL MMSE-DFE for QAM Desired Signal}

In case of PAM signaling, as the desired signal itself is a non-circular random process, WL receivers provide an advantage in both white noise [28], [42] and interference limited situations [31], [32]. However, for QAM signaling, since the desired QAM 
signal is a circular random process, WL receivers can be expected to provide an advantage only when the additive noise is non-circular ${ }^{1}$. In interference limited systems, especially when interference consists of both PAM and QAM components, the noise plus interference signal becomes non-circular. For this type of channel WLF can be applied to enhance system performance. Recall that the time domain signal for QAM signaling is given by $(2.7)$ as:

$$
\tilde{\mathbf{y}}_{k}=\sum_{l=0}^{v} \tilde{\mathbf{H}}_{l} \tilde{\mathbf{x}}_{k-l}+\tilde{\mathbf{n}}_{k}
$$

where the channel takes a $2 s N \times 2$ matrix of form:

$$
\tilde{\mathbf{H}}_{l}=\left[\begin{array}{cc}
\operatorname{Re}\left(\mathbf{h}_{l}\right) & -\operatorname{Im}\left(\mathbf{h}_{l}\right) \\
\operatorname{Im}\left(\mathbf{h}_{l}\right) & \operatorname{Re}\left(\mathbf{h}_{l}\right)
\end{array}\right]
$$

and the modulation takes a vector form: $\tilde{\mathbf{x}}_{k}=\left[\operatorname{Re}\left(x_{k}\right), \operatorname{Im}\left(x_{k}\right)\right]^{\prime}$. In this case, we view the real and imaginary parts of the QAM symbol as two input signals and define the equalizer accordingly. As shown in Figure 4.1, the proposed receiver consists of a matrix-valued feed-forward filter $(\mathrm{FFF}) \tilde{\mathbf{W}}_{k}$, a matrix-valued feedback filter (FBF) $\tilde{\mathbf{B}}_{k}$ and a decision device. The feed-forward and feedback sections are designed such that a decision device will be able to deliver symbol-by-symbol decisions after ISI cancellation with aid of past symbol decisions. Let us define a vector-valued ISI free error signal:

$$
\tilde{\mathbf{e}}_{k} \triangleq\left[e_{I, k}, e_{Q, k}\right]^{\prime}=\tilde{\mathbf{W}}_{k} \circledast \tilde{\mathbf{y}}_{k}-\tilde{\mathbf{B}}_{k} \circledast \tilde{\mathbf{x}}_{k} .
$$

The FFF $\tilde{\mathbf{W}}_{k}$ can be written explicitly as:

$$
\tilde{\mathbf{W}}_{k}=\left[\tilde{\mathbf{W}}_{k, 1}, . ., \tilde{\mathbf{W}}_{k, 2 s N}\right]
$$

\footnotetext{
${ }^{1}$ Since AWGN is a circular random process, WL QAM equalization in case of white noise cannot provide any additional benefit.
} 
where the $i$ the component takes the matrix form:

$$
\tilde{\mathbf{W}}_{k, i}=\left[\begin{array}{cc}
w_{k, i}^{I, I} & w_{k, i}^{I, Q} \\
w_{k, i}^{Q, I} & w_{k, i}^{Q, Q}
\end{array}\right]
$$

due to I-Q split. Note that FFF is matrix filter of dimension $2 \times 2 s N$. We constrain the feedback filter as: $\tilde{\mathbf{B}}_{0}=\mathbf{I}, \tilde{\mathbf{B}}_{k}=0, k<0$, where each tap is a $2 \times 2$ matrix:

$$
\tilde{\mathbf{B}}_{k}=\left[\begin{array}{cc}
b_{k}^{I, I} & b_{k}^{I, Q} \\
b_{k}^{Q, I} & b_{k}^{Q, Q}
\end{array}\right]
$$

The optimum FFF and FBF will be calculated by minimizing either the trace or determinant of the error covariance matrix as:

$$
\left[\tilde{\mathbf{W}}_{k}, \tilde{\mathbf{B}}_{k}\right]^{o p t}=\min _{\left[\tilde{\mathbf{W}}_{k}, \tilde{\mathbf{B}}_{k}\right]} E\left[\tilde{\mathbf{e}}_{k} \tilde{\mathbf{e}}_{k}^{\dagger}\right]
$$

In D-domain:

$$
\tilde{\mathbf{e}}(D)=\tilde{\mathbf{W}}(D) \tilde{\mathbf{y}}(D)-\tilde{\mathbf{B}}(D) \tilde{\mathbf{x}}(D) .
$$

By applying orthogonality principle $E\left[\tilde{\mathbf{e}}(D) \tilde{\mathbf{y}}^{\dagger}\left(D^{-*}\right)\right]=0$, using (4.2), we can solve for the FFF as:

$$
\begin{aligned}
\tilde{\mathbf{W}}(D) & =\tilde{\mathbf{B}}(D) \mathbf{R}_{\tilde{\mathbf{x}} \tilde{\mathbf{y}}}(D) \mathbf{R}_{\tilde{\mathbf{y}} \tilde{\mathbf{y}}}^{-\mathbf{1}}(D) \\
& =\tilde{\mathbf{B}}(D) \mathbf{R}_{\tilde{\mathbf{x}} \tilde{\mathbf{x}}}(D) \tilde{\mathbf{H}}^{\dagger}\left(D^{-*}\right)\left[\tilde{\mathbf{H}}(D) \mathbf{R}_{\tilde{\mathbf{x}} \tilde{\mathbf{x}}}(D) \tilde{\mathbf{H}}^{\dagger}\left(D^{-*}\right)+\mathbf{R}_{\tilde{\mathbf{n}} \tilde{\mathbf{n}}}(D)\right]^{-1} \\
& =\tilde{\mathbf{B}}(D)\left[\mathbf{R}_{\tilde{\mathbf{x}} \tilde{\mathbf{x}}}^{-1}(D)+\tilde{\mathbf{H}}^{\dagger}\left(D^{-*}\right) \mathbf{R}_{\tilde{\mathbf{n}} \tilde{\mathbf{n}}}^{-1}(f) \tilde{\mathbf{H}}(D)\right]^{-1} \tilde{\mathbf{H}}^{\dagger}\left(D^{-*}\right) \mathbf{R}_{\tilde{\mathbf{n}} \tilde{\mathbf{n}}}^{-1}(D)
\end{aligned}
$$

where

$$
\begin{aligned}
& \mathbf{R}_{\tilde{\mathbf{x}} \tilde{\mathbf{y}}}(D) \triangleq \mathbf{R}_{\tilde{\mathbf{x}} \tilde{\mathbf{x}}}(D) \tilde{\mathbf{H}}^{\dagger}\left(D^{-*}\right) \\
& \mathbf{R}_{\tilde{\mathbf{y}} \tilde{\mathbf{y}}}(D) \triangleq \tilde{\mathbf{H}}(D) \mathbf{R}_{\tilde{\mathbf{x}} \tilde{\mathbf{x}}}(D) \tilde{\mathbf{H}}^{\dagger}\left(D^{-*}\right)+\mathbf{R}_{\tilde{\mathbf{n}} \tilde{\mathbf{n}}}(D) \\
& \mathbf{R}_{\tilde{\mathbf{x}} \tilde{\mathbf{x}}}(D) \triangleq E\left[\tilde{\mathbf{x}}(D) \tilde{\mathbf{x}}^{\dagger}\left(D^{-*}\right)\right]=\frac{\mathbf{I}}{2} \\
& \mathbf{R}_{\tilde{\mathbf{n}} \tilde{\mathbf{n}}}(D) \triangleq E\left[\tilde{\mathbf{n}}(D) \tilde{\mathbf{n}}^{\dagger}\left(D^{-*}\right)\right]
\end{aligned}
$$


In the above expressions (4.5) is obtained by using matrix inversion lemma. As we assume that the received signal is corrupted by a sum of $M_{1}$ PAM and $M_{2}$ QAM interferers and additive thermal noise, using $(2.25), \mathbf{R}_{\tilde{\mathbf{n}} \tilde{\mathbf{n}}}(D)$ can be explicitly written in terms of interference correlation matrix as:

$$
\mathbf{R}_{\tilde{\mathbf{n}} \tilde{\mathbf{n}}}(D)=\underbrace{\sum_{l=1}^{M_{1}} \tilde{\mathbf{g}}^{l}(D) \tilde{\mathbf{g}}^{l \dagger}\left(D^{-*}\right)}_{\text {PAM CCI contribution }}+\underbrace{\frac{1}{2} \sum_{m=1}^{M_{2}} \tilde{\mathbf{G}}^{m}(D) \tilde{\mathbf{G}}^{m \dagger}\left(D^{-*}\right)}_{\text {QAM CCI contribution }}+\frac{s N_{0}}{2} \mathbf{I} .
$$

To solve for the feedback filter $\tilde{\mathbf{B}}(D)$, the matrix-valued covariance process is factored as:

$$
\left[\mathbf{R}_{\tilde{\mathbf{x}} \tilde{\mathbf{x}}}^{-1}(D)+\tilde{\mathbf{H}}^{\dagger}\left(D^{-*}\right) \mathbf{R}_{\tilde{\mathbf{n}} \tilde{\mathbf{n}}}^{-1}(D) \tilde{\mathbf{H}}(D)\right]=\tilde{\mathbf{U}}^{\dagger}\left(D^{-*}\right) \Upsilon \tilde{\mathbf{U}}(D)
$$

where $\tilde{\mathbf{U}}^{\dagger}\left(D^{-*}\right)$ is a causal, monic, stable matrix filter with a causal inverse [43]. The determinant of $\Upsilon$ is given by:

$$
|\Upsilon|=e^{T \int_{-\frac{1}{2 T}}^{\frac{1}{2 T}} \ln \left|\left[\mathbf{R}_{\tilde{\mathbf{x}} \mathbf{x}}^{-1}(f)+\tilde{\mathbf{H}}^{\dagger}(-f) \mathbf{R}_{\tilde{\mathbf{n}}}^{-1}(f) \tilde{\mathbf{H}}(f)\right]\right| d f} .
$$

It can be further shown that (see [44], [45]) both arithmetic $\left(\operatorname{Tr}\left[\mathbf{R}_{\tilde{e} \tilde{e}}\right]\right)$ and geometric $\left(\left|\mathbf{R}_{\tilde{e} \tilde{e}}\right|\right)$ mean square error metrics are minimized by choosing: $\tilde{\mathbf{B}}(D)=\tilde{\mathbf{U}}(D)$. Using this, the optimum FFF can be expressed as:

$$
\tilde{\mathbf{W}}(D)=[\Upsilon]^{-1} \tilde{\mathbf{U}}^{\dagger-\mathbf{1}}\left(D^{-*}\right) \tilde{\mathbf{H}}^{\dagger}\left(D^{-*}\right) \mathbf{R}_{\tilde{\mathbf{n}} \tilde{\mathbf{n}}}^{-\mathbf{1}}(D) .
$$

The corresponding minimum GMSE becomes:

$$
\operatorname{GMSE}_{\text {WL-DFE QAM }}=\left|\mathbf{R}_{\tilde{\mathbf{e}} \tilde{e}}\right|=|\Upsilon|^{-1}=e^{-T \int_{-\frac{1}{2 T}}^{\frac{1}{2 T}} \ln \left|\left[\mathbf{R}_{\tilde{\mathbf{x}} \mathbf{\tilde { x }}}^{-1}(f)+\tilde{\mathbf{H}}^{\dagger}(-f) \mathbf{R}_{\tilde{\mathbf{n}} \tilde{\tilde{n}}}^{-1}(f) \tilde{\mathbf{H}}(f)\right]\right| d f} .
$$

The optimum FBF and FBF settings can be determined using state-space methods of $[40]$.

\subsubsection{Symbol Detection}

In conventional systems (and also in PAM WL-DFE case), the DFE slicer takes a scalar signal as input and delivers symbol decisions using standard slicing (threshold 
detection) techniques. However, the WL QAM receiver, as shown in Figure 4.1, has to deal with two (real-valued) inputs corresponding to the I-Q branches. Ignoring the receiver bias and assuming that the residual noise at the input to the decision device has multi-variate Gaussian pdf ${ }^{2}$ of form:

$$
p\left(\tilde{\mathbf{e}}_{k}\right)=\frac{1}{2 \pi \sqrt{|\Upsilon|}} e^{-\left(\tilde{\mathbf{e}}_{k}^{\dagger} \Upsilon^{-1} \tilde{\mathbf{e}}_{k}\right)}
$$

the ML DFE decision rule can be formulated as:

$$
d_{\tilde{\mathbf{x}}_{k}}=\arg \min _{\tilde{\mathbf{x}}_{k}}\left[\tilde{\mathbf{z}}_{k}-\overline{\mathbf{B}}_{k} \circledast \hat{\mathbf{x}}_{k}-\tilde{\mathbf{x}}_{k}\right]^{\dagger} \Upsilon^{-1}\left[\tilde{\mathbf{z}}_{k}-\overline{\mathbf{B}}_{k} \circledast \hat{\mathbf{x}}_{k}-\tilde{\mathbf{x}}_{k}\right]
$$

where $\tilde{\mathbf{z}}_{k}=\tilde{\mathbf{W}}_{k} \circledast \tilde{\mathbf{y}}_{k}$ is the feed-forward filtered signal, $\overline{\mathbf{B}}_{k}$ is the feedback filter with the first tap set to 0 , and $\hat{\mathbf{x}}_{k}$ contains the I-Q parts of the past symbol estimates. The DFE receiver selects the argument that minimizes the minimum distance over all symbol possibilities.

\subsubsection{WL LE for QAM}

The MMSE-DFE structure assumes a linear form (see Figure 4.2) if the feedback filter is constrained to be an identity matrix. In this case, the receiver consists of a FFF followed by a symbol detector. The optimum FFF is given by:

$$
\tilde{\mathbf{W}}(D)=\left[\mathbf{R}_{\tilde{\mathbf{x}} \tilde{\mathbf{x}}}^{-\mathbf{1}}(D)+\tilde{\mathbf{H}}^{\dagger}\left(D^{-*}\right) \mathbf{R}_{\tilde{\mathbf{n}} \tilde{\mathbf{n}}}^{-\mathbf{1}}(D) \tilde{\mathbf{H}}(D)\right]^{-1} \tilde{\mathbf{H}}^{\dagger}\left(D^{-*}\right) \mathbf{R}_{\tilde{\mathbf{n}} \tilde{\mathbf{n}}}^{-\mathbf{1}}(D)
$$

and the MSE can be expressed as:

$$
\operatorname{GMSE}_{\mathrm{WL}-\mathrm{LE} \text { QAM }}=\left|\mathbf{R}_{\tilde{\mathbf{e}} \tilde{\mathbf{e}}}\right|=\left|T \int_{-\frac{1}{2 T}}^{\frac{1}{2 T}}\left[\mathbf{R}_{\tilde{\mathbf{x}} \tilde{\mathbf{x}}}^{-\mathbf{1}}(f)+\tilde{\mathbf{H}}^{\dagger}(-f) \mathbf{R}_{\tilde{\mathbf{n}} \tilde{\mathbf{n}}}^{-\mathbf{1}}(f) \tilde{\mathbf{H}}(f)\right]^{-1} d f\right| .
$$

In this case, the decision device uses a modified branch metric of form:

$$
d_{\tilde{\mathbf{x}}_{k}}=\arg \min _{\tilde{\mathbf{x}}_{k}}\left[\tilde{\mathbf{z}}_{k}-\tilde{\mathbf{x}}_{k}\right]^{\dagger} \mathbf{R}_{\tilde{\mathbf{e}} \mathbf{e}}^{-1}\left[\tilde{\mathbf{z}}_{k}-\tilde{\mathbf{x}}_{k}\right]
$$

\footnotetext{
${ }^{2}$ Although interference is non-Gaussian (the modulation alphabets of CCI have binomial distribution), the Gaussian approximation leads to simple decision metric and provides excellent BER results
} 
where $\tilde{\mathbf{z}}_{k}=\tilde{\mathbf{W}}_{k} \circledast \tilde{\mathbf{y}}_{k}$ is the feed-forward filtered signal.

Remark If the interference correlation matrix has non-zero correlation among I-Q branches (e.g. in case of PAM-type interference), the IC process will be split into two phases. The front-end noise whitening filter $\mathbf{R}_{\tilde{\mathbf{n}} \tilde{\mathbf{n}}}^{-1}(D)$ portion of the FFF first offers a partial IC effect while the remaining IC gain is extracted by the symbol detector that takes into account the residual correlations between the I-Q branches. When the correlation between I-Q branches is zero (e.g. when all the interfering signals have QAM or AWGN case) the front-end noise whitening filter is solely responsible for the IC gain. The symbol wise noise whitener $\Upsilon^{-1}$ has no role to play in this case as it becomes a scaled identity matrix.

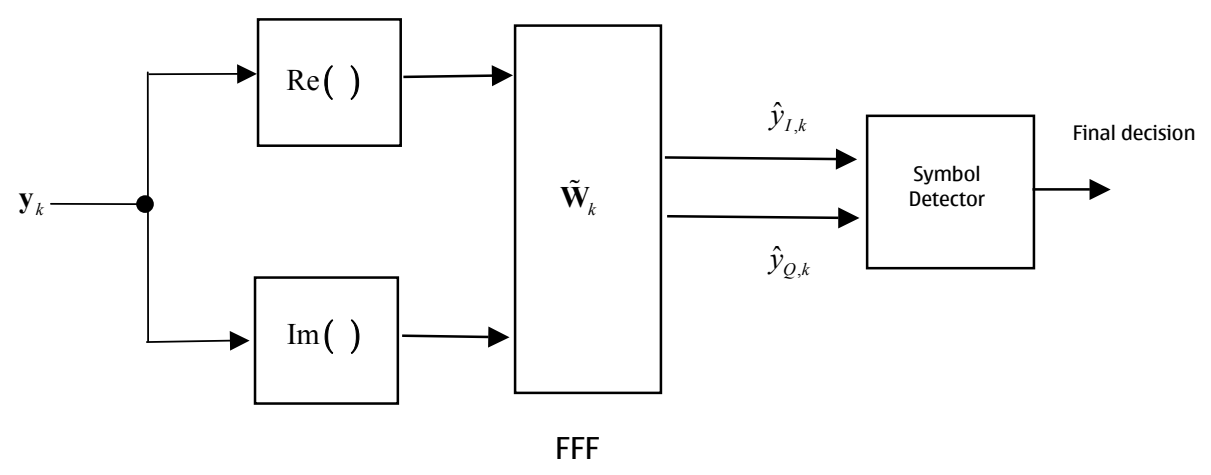

Figure 4.2. WL LE for QAM.

\subsection{Interference Cancellation Properties}

The receiver structures proposed in the previous section combined with the receiver concepts proposed in Chapter 3 completes the WL receiver development for both PAM and QAM systems with ISI and CCI. In this section we provide a direct comparison between the IC properties of conventional and WL methods. In Section 4.3.1, we first compare conventional and WL receivers for DFE case followed by an analysis 
for $\mathrm{LE}$ in 4.3.2. In particular, we determine the IC properties for the interference model given in Section 2.4 where we modeled the system with a mixture of $M_{1}$ PAM interferers and $M_{2}$ QAM interferers. As we are interested in determining the IC gain provided by antennas alone, in the rest of the analysis, we ignore the possible side effects caused by transmit/receiver filters by assuming an over-sampling factor $s=1$. Also, we would like to remark here that the IC analysis of this section has similarities to the rank analysis used by Winters et al. in [17] for conventional IC systems.

\subsubsection{Comparison between Conventional and WL DFE Receivers}

Using (3.41), (4.7), (3.47), the SNR at the output of a DFE receiver for conventional, WL QAM and WL PAM cases is given by:

$$
\begin{aligned}
\operatorname{SNR}_{\text {Conv DFE }} & \triangleq \frac{1}{\operatorname{MSE}_{\text {Conv DFE }}}=e^{T \int_{-\frac{1}{2 T}}^{\frac{1}{2 T}} \ln \left[R_{x x}^{-1}(f)+\mathbf{h}^{\dagger}(-f) \mathbf{R}_{\mathbf{n}}^{-1}(f) \mathbf{h}(f)\right] d f} \\
\operatorname{GSNR}_{\text {WL-DFE QAM }} & \triangleq \frac{1}{\operatorname{GMSE}_{\text {WL-DFE QAM }}}=e^{T \int_{-\frac{1}{2 T}}^{\frac{1}{2 T}} \ln \left|\left[\mathbf{R}_{\tilde{\mathbf{x}} \tilde{\mathbf{x}}}^{-1}(f)+\tilde{\mathbf{H}}^{\dagger}(-f) \mathbf{R}_{\tilde{\mathbf{n}} \tilde{\mathbf{n}}}^{-1}(f) \tilde{\mathbf{H}}(f)\right]\right| d f} \\
\operatorname{SNR}_{\text {WL-DFE PAM }} & \triangleq \frac{1}{\operatorname{MSE}_{\mathrm{WL}-\mathrm{DFE} \mathrm{PAM}}}=e^{T \int_{-\frac{1}{2 T}}^{\frac{1}{2 T}} \ln \left[R_{x x}^{-1}(f)+\tilde{\mathbf{h}}^{\dagger}(-f) \mathbf{R}_{\tilde{\mathbf{n}} \tilde{\mathbf{n}}}^{-1}(f) \tilde{\mathbf{h}}(f)\right] d f}
\end{aligned}
$$

where

$$
\begin{aligned}
& \mathbf{R}_{\mathbf{n} \mathbf{n}}(D)=\sum_{m=1}^{M} \mathbf{g}^{m}(D) \mathbf{g}^{m \dagger}\left(D^{-*}\right)+N_{0} \mathbf{I} \\
& \mathbf{R}_{\tilde{\mathbf{n}} \tilde{\mathbf{n}}}(D)=\underbrace{\sum_{l=1}^{M_{1}} \tilde{\mathbf{g}}^{l}(D) \tilde{\mathbf{g}}^{l \dagger}\left(D^{-*}\right)}_{\text {PAM CCI contribution }}+\underbrace{\frac{1}{2} \sum_{m=1}^{M_{2}} \tilde{\mathbf{G}}^{m}(D) \tilde{\mathbf{G}}^{m \dagger}\left(D^{-*}\right)}_{\text {QAM CCI contribution }}+\frac{N_{0}}{2} \mathbf{I}
\end{aligned}
$$

denotes the D-domain noise plus interference correlation matrices for conventional and I-Q cases respectively. These expressions are derived in Section 2.4. The corresponding frequency domain quantities can be obtained by letting $D=e^{-j 2 \pi f T}$. 
Also, note that the above SNR metrics are obtained for an ideal DFE receiver whose feedback path is assumed to be error free. Let

$$
\begin{aligned}
\gamma(f) & \triangleq \mathbf{h}^{\dagger}(-f) \mathbf{R}_{\mathbf{n} \mathbf{n}}^{-\mathbf{1}}(f) \mathbf{h}(f) \quad \text { Conv } \\
& \triangleq\left|\tilde{\mathbf{H}}^{\dagger}(-f) \mathbf{R}_{\tilde{\mathbf{n}} \tilde{\mathbf{n}}}^{-1}(f) \tilde{\mathbf{H}}(f)\right| \quad \text { WL QAM } \\
& \triangleq \tilde{\mathbf{h}}^{\dagger}(-f) \mathbf{R}_{\tilde{\mathbf{n}} \tilde{\mathbf{n}}}^{-1}(f) \tilde{\mathbf{h}}(f) \quad \text { WL PAM }
\end{aligned}
$$

denote the frequency dependent SNR term. We adopt the following criterion. We say that the receiver provides full IC gain i.e. complete interference suppression if

$$
\lim _{N_{0} \rightarrow 0} \gamma(f) \rightarrow \infty .
$$

If we substitute this result in (4.11), (4.12) and (4.13) we can see that the considered DFE receivers offer an infinite SNR gain irrespective of interference power level. This usually happens when the interference correlation matrix of a given receiver becomes rank deficient. Using the derivations given in Appendix C.1 first we show that, under the assumption of ideal decision feedback, a conventional receiver with $N$ antennas and $M$ interferers $\left(M=M_{1}+M_{2}, M<N\right)$ can suppress $N-M$ co-channel interferers. When $M \geq N$, the receiver can not offer full IC gain but gives a marginal IC benefit. Note that this result is independent of modulation used by the signal or interference. On the other hand, for the WL case, in Appendix C.2 we showed that, under the assumption of ideal decision feedback, a QAM WL-DFE receiver equipped with $N$ antennas can fully reject any combination of $M_{1}$ PAM and $M_{2}$ QAM interferers that satisfy the constraint: $M_{1}+2 M_{2}<2 N$. As PAM can be viewed as a special case of QAM, this result applies to PAM WL-DFE as well. To understand the IC benefit, we compare the IC ability of conventional and (PAM/QAM) WL receivers in Table 4.1. In this table, for conventional scenario, we showed the maximum number of interferers $\left(M_{\max }=N-1\right)$ the receiver can potentially suppress, whereas for WL 
case we showed all possible combinations of PAM and QAM interferers $\left(M_{1}, M_{2}\right)$ that satisfy the constraint $M_{1}+2 M_{2}<2 N$. For example, for single and dual antenna cases:

- a single antenna WL MMSE-DFE receiver can suppress a single PAM interferer

- a dual antenna receiver can suppress either 3 PAM interferers or a total of two mixed (one PAM plus one QAM) interferers

In contrast, a conventional receiver:

- has no IC capability with a single antenna

- with two antennas, it can reject a single interferer only.

Essentially, when interference consists of PAM or a mixture of PAM and QAM signals, the WL-DFE receiver suppresses a higher number of interferers than conventional receivers. In practice, while the stated results are true in a flat (ISI free) fading channel, in frequency selective fading, simulation showed that, decision feedback errors often prevents the QAM WL-DFE to reach its full IC potential whereas the PAM WL-DFE provides full IC gain independent of the channel model. Therefore, for WL receivers, the modulation type used by the desired signal or interference plays a significant role in determining the gain ${ }^{3}$.

\subsubsection{Comparison between Conventional and WL LE Methods}

In case of LE, using (3.5), (3.9) we can express the SNR at the output of LE as

$$
\begin{aligned}
\mathrm{SNR}_{\text {Conv LE }} \triangleq \frac{1}{\mathrm{MSE}_{\text {Conv LE }}}=\left[T \int_{-\frac{1}{2 T}}^{\frac{1}{2 T}} \frac{1}{\left[1+\mathbf{h}^{\dagger}(-f) \mathbf{R}_{\mathbf{n n}}^{-\mathbf{1}(f) \mathbf{h}(f)]} d f\right]^{-1}}\right. \\
\mathrm{SNR}_{\mathrm{WL}-\mathrm{LE} \text { PAM }} \triangleq \frac{1}{\mathrm{MSE}_{\mathrm{WL}-\mathrm{LE} \mathrm{PAM}}}=\left[T \int_{-\frac{1}{2 T}}^{\frac{1}{2 T}} \frac{1}{\left[1+\tilde{\mathbf{h}}^{\dagger}(-f) \mathbf{R}_{\tilde{\mathbf{n}} \tilde{\mathbf{n}}}^{-\mathbf{1}}(f) \tilde{\mathbf{h}}(f)\right]} d f\right]^{-1} .
\end{aligned}
$$

\footnotetext{
${ }^{3}$ We would like to mention that WL methods provide an IC advantage in the presence of PAMtype interference only. In the absence of PAM-interference, conventional and WL methods have same IC ability.
} 
Table 4.1. IC comparison between conventional and WL receivers

\begin{tabular}{|c|c|c|}
\hline$N$ & $\begin{array}{c}\text { Conv } \\
M_{\max }\end{array}$ & $\begin{array}{c}\text { WL } \\
\left(M_{1}, M_{2}\right)\end{array}$ \\
\hline 1 & 0 & $(1,0)$ \\
\hline 2 & 1 & $(1,1),(3,0)$ \\
\hline 3 & 2 & $(1,2),(3,1),(5,0)$ \\
\hline 4 & 3 & $(1,3),(3,2),(5,1),(7,0)$ \\
\hline
\end{tabular}

For the above two cases, using the infinite SNR criterion (4.19), we can show that both conventional LE and PAM WL-LE provide full IC gain when the respective ICM is rank deficient (see Appendix C.1, C.2 for details). Hence the IC properties shown in Table 4.1 apply to the conventional and PAM WL LE receivers. However, the QAM WL-LE does not follow this trend. Using (4.10), let us consider the GSNR for a QAM WL-LE receiver

$$
\begin{aligned}
\operatorname{GSNR}_{\mathrm{WL}-\mathrm{LE} Q \mathrm{QAM}} & \triangleq \frac{1}{\mathrm{GMSE}_{\mathrm{WL}-\mathrm{LE} Q \mathrm{QM}}} \\
& =\left[\left|T \int_{-\frac{1}{2 T}}^{\frac{1}{2 T}}\left[\mathbf{R}_{\tilde{\mathbf{x}} \tilde{\mathbf{x}}}^{-\mathbf{1}}(f)+\tilde{\mathbf{H}}^{\dagger}(-f) \mathbf{R}_{\tilde{\mathbf{n}} \tilde{\mathbf{n}}}^{-\mathbf{1}}(f) \tilde{\mathbf{H}}(f)\right]^{-1} d f\right|\right]^{-1}
\end{aligned}
$$

Even when $\left|\tilde{\mathbf{H}}^{\dagger}(-f) \mathbf{R}_{\tilde{\mathbf{n}} \tilde{\mathbf{n}}}^{-\mathbf{1}}(f) \tilde{\mathbf{H}}(f)\right|$ becomes infinity, the GSNR expression given in (4.20) need not necessarily become infinity. In this case, it is not straightforward to explain the IC gain through rank analysis as in QAM WL-DFE case. Simulation showed that, this method does not provide full IC gain whenever the channel has frequency selectivity. The reason could be attributed to noise enhancement of matrix-valued equalizer that has to equalize the signal in I-Q and temporal directions simultaneously. 


\subsection{Complexity Aspects}

In Chapter 3, we have shown that WL receivers provide both performance and complexity advantage for PAM signals when the noise is AWGN. However, when the wanted signal has QAM modulation, we can show that (see Appendix D.1) the MSE is same for both conventional and WL receivers. It is also possible to show that FFF and FBF tap values are identical in the sense that if the conventional filter taps have complex values $w_{k}$, the corresponding WL filters take same values except that they are arranged in matrix form as: $\left[\begin{array}{cc}\operatorname{Re}\left(w_{k}\right) & -\operatorname{Im}\left(w_{k}\right) \\ \operatorname{Im}\left(w_{k}\right) & \operatorname{Re}\left(w_{k}\right)\end{array}\right]$. Therefore, the computational complexity is also same for WL and conventional receivers with QAM signaling in case of white noise. But there are significant complexity differences in the presence of CCI.

\subsubsection{Complexity of WL-PAM Receiver}

In interference limited systems, all the MMSE receivers use a front-end noise whitening filter to suppress interference (for reference see the FFF expressions given in (3.4), (3.8) for conventional and WL LE cases). In conventional case, the noise whitening filter $\mathbf{R}_{\mathbf{n n}}^{-\mathbf{1}}(f)$ has a dimension of $N \times N$ (assuming $s=1$ ) whereas the I-Q whitening filter $\mathbf{R}_{\tilde{\mathbf{n}} \tilde{\mathbf{n}}}^{-\mathbf{1}}(f)$ has a larger dimensionality $2 N \times 2 N$. The higher dimensionality implies a complexity increase. However, after the noise whitening step, since the residual noise is white, the MF and MMSE filter implementation is simpler for WL case (see Section 3.2.4 for details). In general, the complexity differences in PAM case depend highly on the type of channel under consideration. 


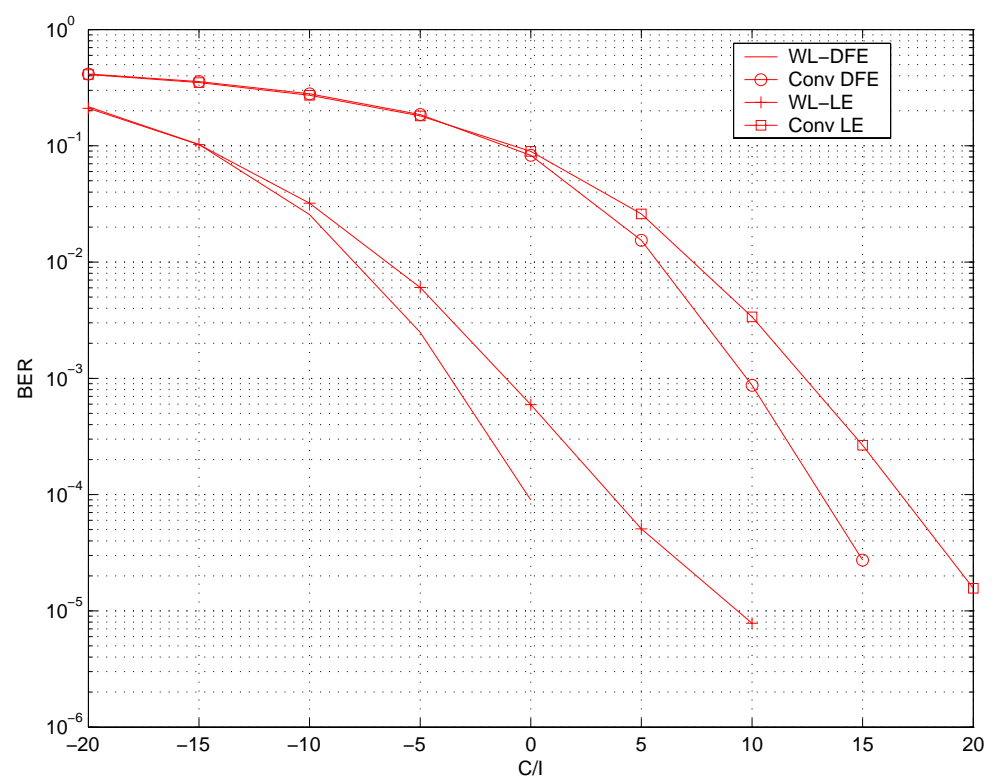

Figure 4.3. Single antenna BPSK detection with single BPSK interferer.

\subsubsection{Complexity of WL-QAM Receiver}

When the wanted signal has QAM modulation, the FFF and FBF become MIMO filters that have twice the size of conventional filters. Hence, the calculation and implementation of the noise whitening filter and the rest of the filters (FFF, FBF) are more complex for WL case. Moreover, the WL receiver has to calculate matrix spectrum factorization $(\mathrm{SF})$ that is computationally more intensive than conventional scalar SF. In addition, the WL symbol detector requires higher complexity than a standard conventional DFE. Note that a conventional DFE receiver can be implemented with low complexity using slicing techniques whereas the WL demodulator given by (4.8) must explicitly calculate the Euclidean distance metrics for all possible symbol possibilities (at least $M$ distance metrics) to find the minimum metric to make a single symbol decision. 


\subsection{Simulation Results}

In this section we compare the BER performance of WL receivers to conventional systems for both PAM and QAM signaling schemes. In our simulation setup, the wanted signal is modeled as either BPSK or 8-PSK (with Gray coding) and the noise part is modeled as a sum of several co-channel interferers (of equal average power) and thermal noise. The CCI is modeled as a mixture of BPSK and 8-PSK signals. We present simulation results for 5-tap (T-spaced) i.i.d. Rayleigh fading. We assumed ideal channel state information (CSI) and perfect knowledge of ICM at the receiver. Further, the CSI of desired signal and individual interferers are assumed to be statistically independent. The results are reported for interference limited scenarios where the total interference to thermal noise power ratio is set at $20 \mathrm{~dB}$ level. The equalizer is implemented using FIR filters using the FIR MIMO MMSE-DFE method proposed in [45]. We used 17 taps for the FFF and 15 taps for FBF. For LE, FFF length is set to 30 taps. Note that the filter settings are chosen to emulate near infinite length performance. The bit error rate (BER) results are averaged over 2000 channel realizations. We discuss the performance of both single and dual antennas systems. The BER results are plotted as a function of $\mathrm{C} / \mathrm{I}$ that is the total signal to interference power ratio.

\subsubsection{Single Antenna Interference Cancellation}

In Figure 4.3 we show the BER results for a single antenna BPSK receiver in the presence of a single BPSK CCI. In this case, both WL-LE and WL-DFE receivers are able to provide full IC gain. However, with 8-PSK CCI, the BER results given in Figure 4.4 show that WL-LE gives only 1.0-2.0 dB gain over conventional LE while WL-DFE gives about $0.5 \mathrm{~dB}$ over conventional DFE. Note that in the presence of 8-PSK CCI, the WL receiver can not provide any IC gain but the gain we observed 


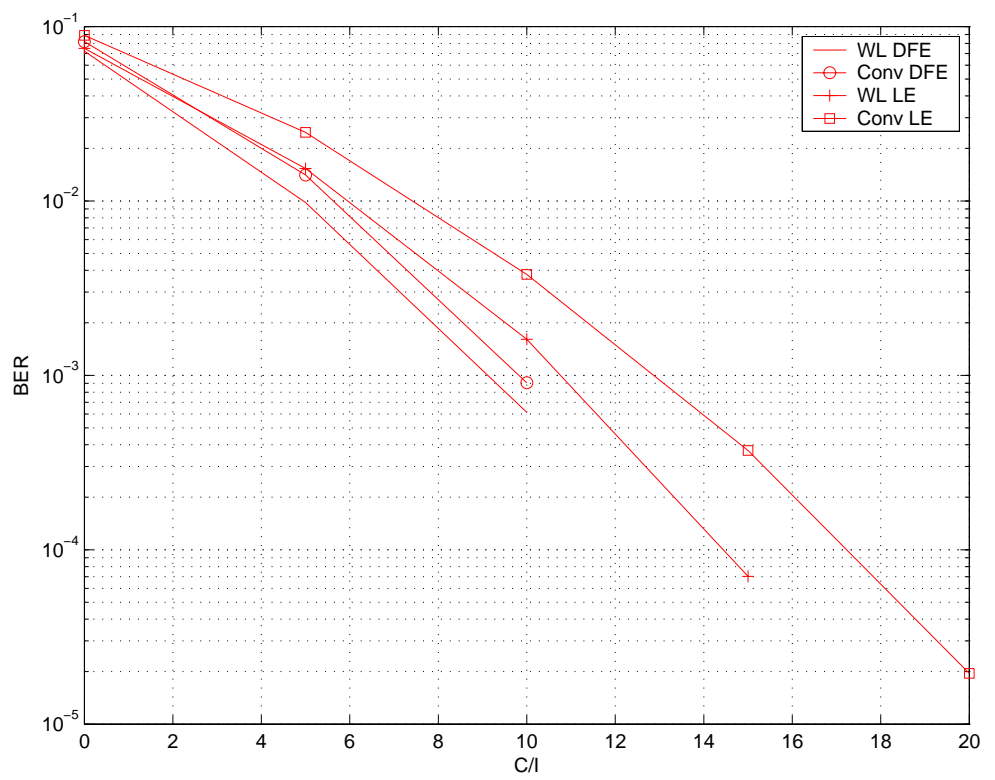

Figure 4.4. Single antenna BPSK detection with single 8-PSK interferer.

in Figure 4.4 is mainly due to lower noise enhancement that is inherent to PAM WL equalizers.

For a single antenna 8-PSK system, in Figure 4.5 we plotted the BER with a single BPSK CCI. In this case, WL-LE did not provide significant gain but WL-DFE gives about $3.0 \mathrm{~dB}$ gain. To understand the limitations of WL-DFE we showed the BER curves with perfect decision feedback. We see that the DFE error propagation is not a serious problem for conventional DFE but it severely limited the gain of WL-DFE. This problem may be alleviated to some extent by using more complex receivers such as RSSE or turbo equalization. Note that WL techniques does not offer any gain for 8-PSK detection in the presence of 8-PSK type interference. In the next section, we show that multi-antenna WL receivers very well can suppress both BPSK and 8-PSK type interference. 


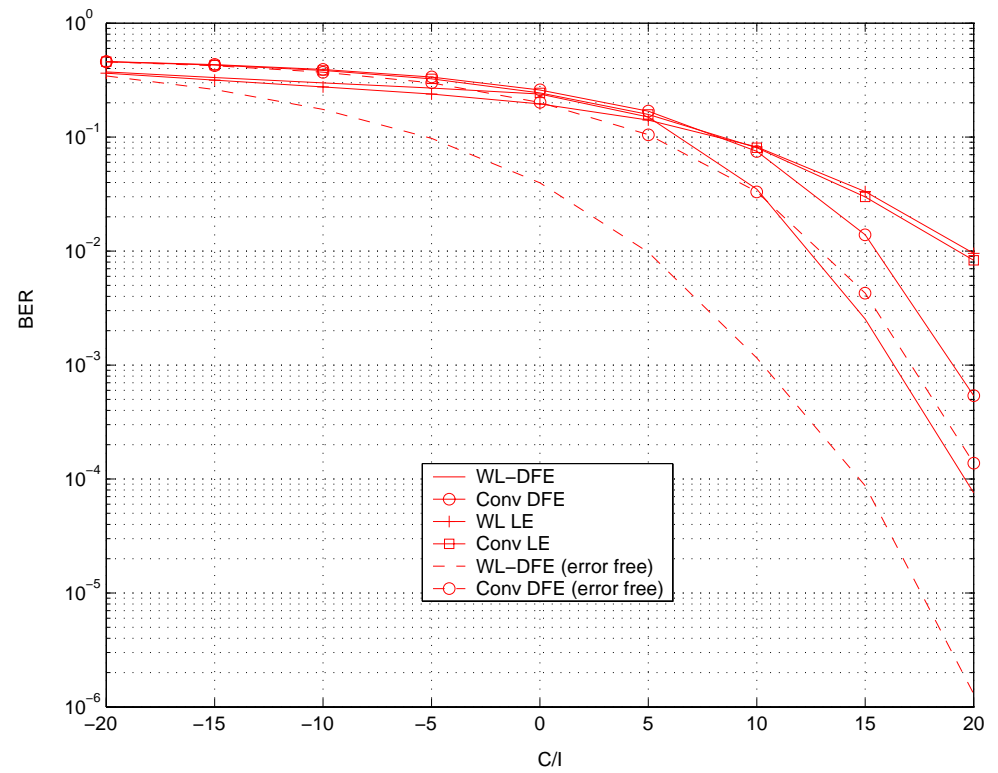

Figure 4.5. Single antenna 8-PSK detection with single BPSK interferer.

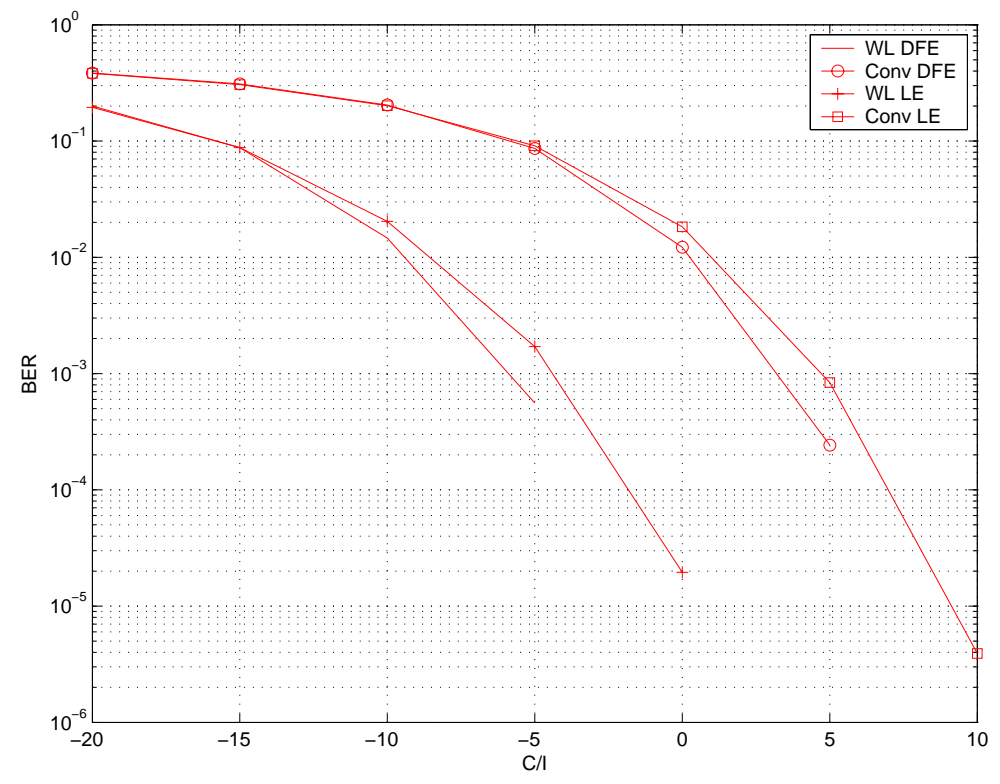

Figure 4.6. Dual antenna BPSK detection with 3-BPSK interferers. 


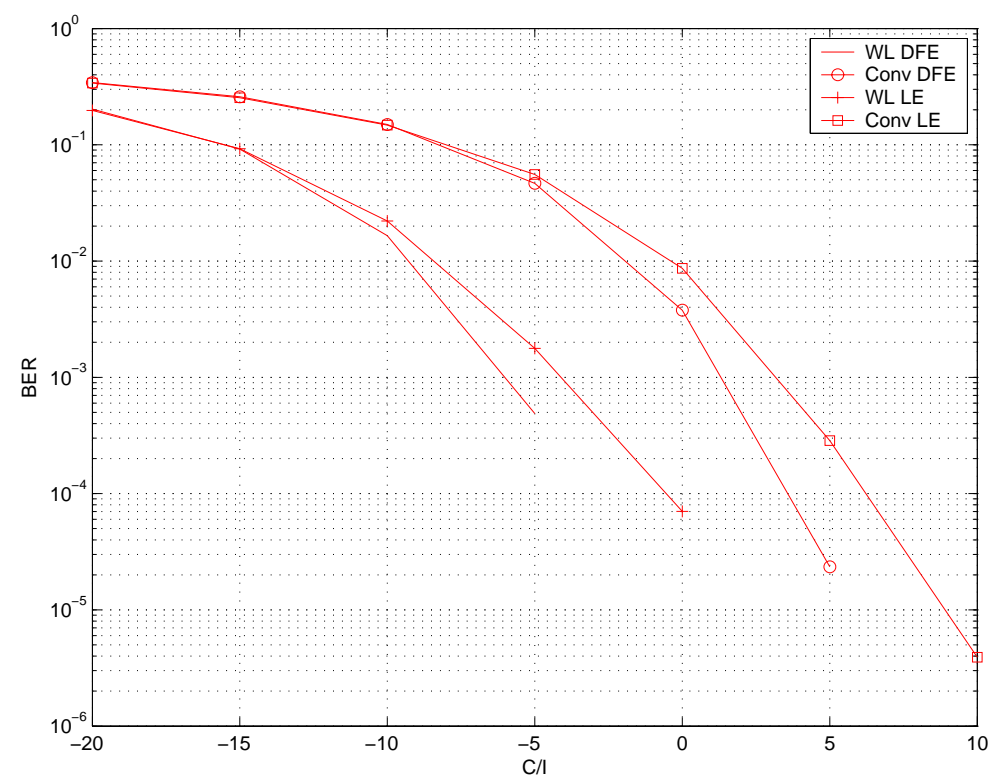

Figure 4.7. Dual antenna BPSK receiver with mixed CCI (PAM+QAM).

\subsubsection{Two Antennas Results}

In Figure 4.6 we show performance of a two antenna BPSK receiver in case of 3-BPSK interferers. As predicted by the WL-DFE and WL-LE analysis the WL method is able to fully reject all 3-interferers in this case. Moreover, the performance difference between LE and DFE methods is fairly small (less than $1.0 \mathrm{~dB}$ ). We also verified in Figure 4.7 that the IC performance is about the same when any two of the 3-BPSK interferers are substituted with an 8-PSK interferer.

As shown in Figure 4.8, for 8-PSK signaling, in case of single BPSK interferer, the 2-antenna WL receivers offer significant gain for both WL-LE and WL-DFE configurations. Recall that the gain was modest (see Figure 4.5) when a single antenna was used. But the gain increased quite significantly with an increase in the number of antennas. With 3-BPSK interferers (see Figure 4.9) the WL-LE gives about 2.0 $\mathrm{dB}$ gain over conventional LE whereas the WL-DFE offers a gain of about $5.0 \mathrm{~dB}$ 


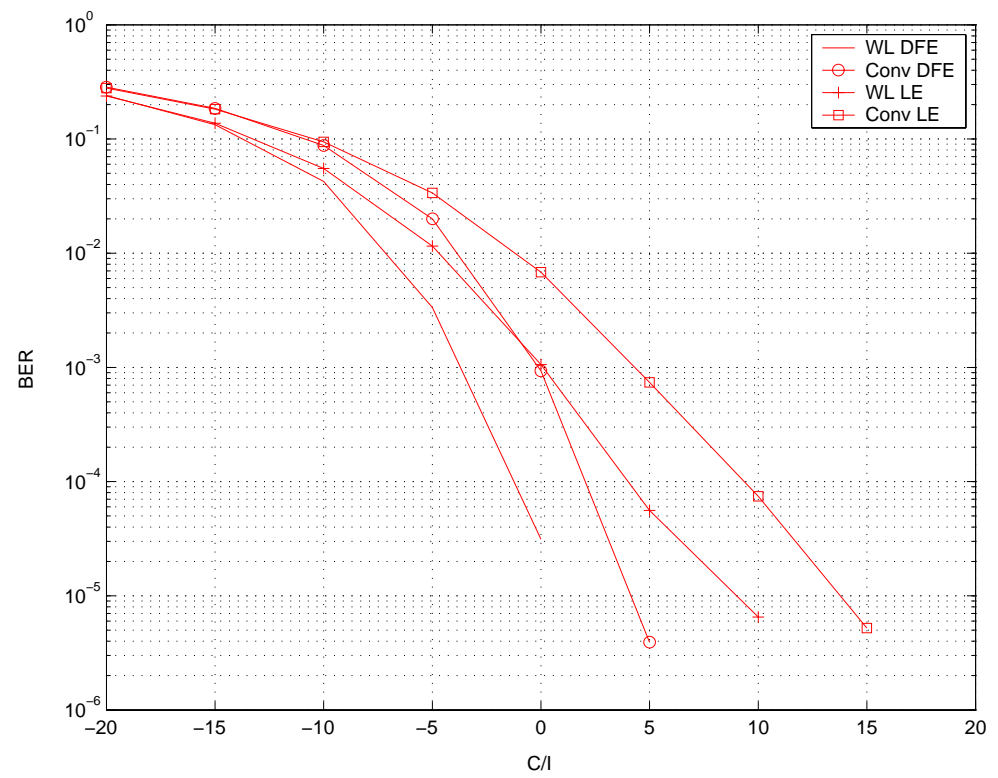

Figure 4.8. Dual antenna 8-PSK detection with single BPSK interferer.

although its IC capability is limited by the error propagation problem. We observed a similar trend in a mixed interference scenario (see Figure 4.10).

\subsection{Summary}

In this chapter we generalized the existing WL equalization structures to include QAM and PAM signaling schemes, multiple antennas with multiple interferers as special cases. Infinite length, WL-DFE and WL-LE receiver structures are proposed and performance of these schemes is analyzed through MSE metrics. Simulations confirmed that, in frequency selective channels, both WL-LE and WL-DFE methods provide full IC benefit (as predicted by the MSE analysis) when the desired signal has PAM. For QAM signaling, in some cases, the DFE error propagation problem prevented the WL-DFE to achieve its full IC potential, while WL-LE suffered from noise enhancement problems. Nevertheless, the advantage of WL detection is significant in most cases of interest. We think that the loss caused by the DFE error 


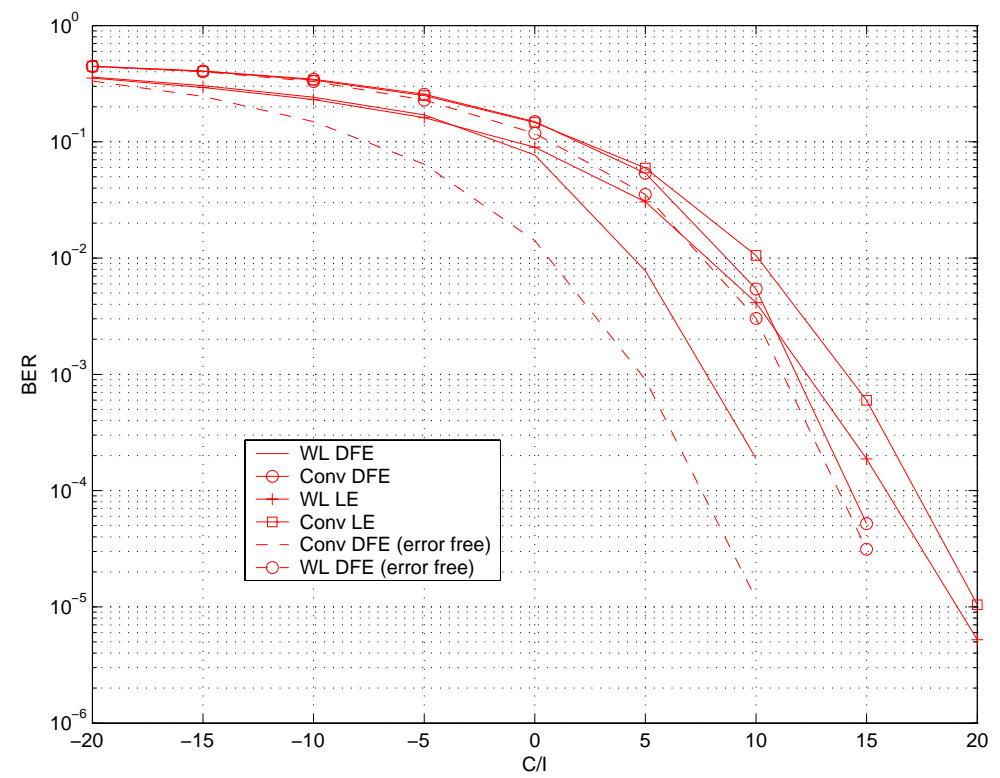

Figure 4.9. Dual antenna 8-PSK detection with 3-BPSK interferers.

propagation problem can be recovered through more complex receivers such as RSSE or turbo equalization. Also, unlike white noise case, WL implementation in CCI limited channels increases receiver complexity. Considering the performance benefits, we think that the additional computational requirements can be justified. 


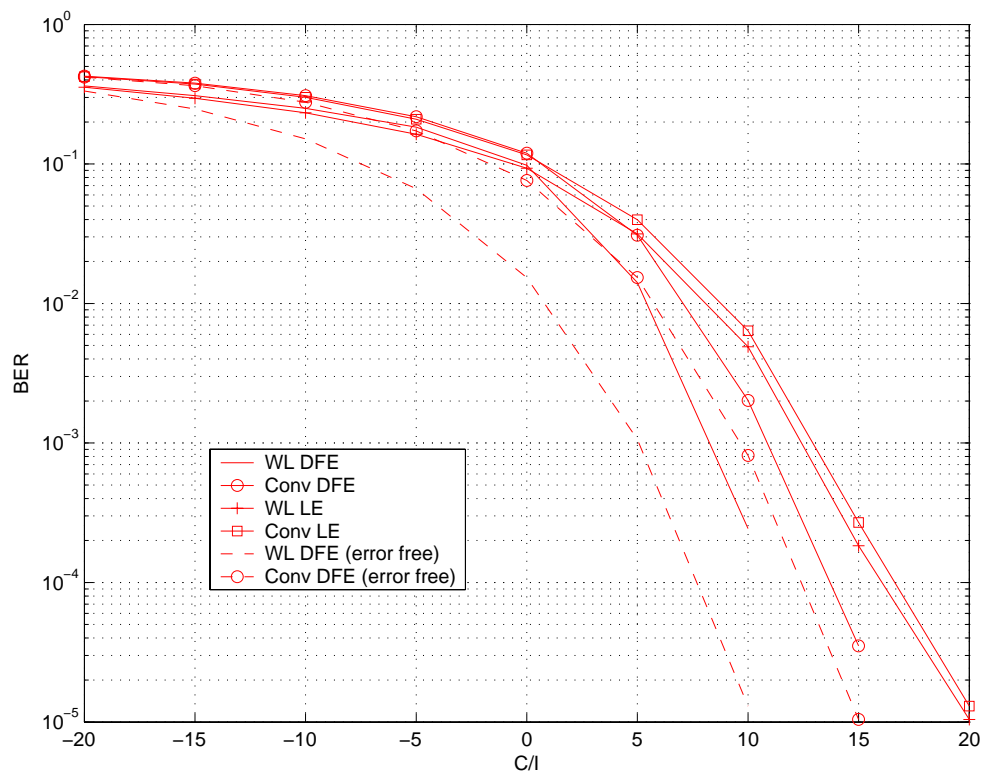

Figure 4.10. Dual antenna 8-PSK receiver with mixed CCI (PAM+QAM). 


\section{CHAPTER 5}

\section{DIVERSITY ANALYSIS}

\subsection{Introduction}

Multiple antennas can enhance performance of wireless systems corrupted by fading and interference. The multi-antenna MMSE receivers that we discussed in the previous chapters basically attempt to maximize the total SNR at the output of the receiver regardless of the channel characteristics i.e. whether the channel is thermal noise or interference limited. In the absence of interference, we can show that the proposed receivers emulate a maximum ratio combing (MRC) receiver and thus gives full diversity benefit from all antennas. In interference limited cases the receiver gives both diversity and IC gain. Especially, when the number of receiver antennas is less than the number of interferers, the MMSE receivers first use the available dimensions (created by antennas or I-Q split) for interference rejection and the remaining dimensions for diversity. In fading channels, as both diversity and interference cancellation features are crucial to good system performance, it is important to understand the relative performance trade-off.

In Chapter 4, the IC performance of WL receivers was analyzed with ISI and CCI. Performance was analyzed by evaluating the MSE at the output of the equalizer. In such cases, analytical evaluation of bit error rate is difficult since the MSE is a complicated function of channel characteristics. However, in the absence of ISI, and when the channel has flat Rayleigh fading, error rate analysis is tractable. In this 
chapter we specifically consider the implementation, SER performance, and diversityIC trade-off of WL receivers in ISI free channels.

In [4], Winters et al. showed that, in flat fading channels, a conventional MMSE receiver with $N$ antennas and $M$ interferers $(M<N)$ can suppress $M$ interferers with a diversity order equal to: $N-M$. Moreover, they obtained SER expressions in [46] for conventional IRC receivers for the single interferer case with flat Rayleigh fading and simulation was used to study performance when interference has multiple co-channel signals with arbitrary power. Recently, in [47], Chiani et al. extended those results for M-PSK systems. They derived tight bounds and approximations for M-PSK with multiple antennas and multiple interferers of equal average power in flat Rayleigh fading channels. But, none of the previous papers considered SER results for WL receivers. Because of differences inherent to conventional and WL methods, the existing conventional SER expressions can not be applied to WL receivers.

In this chapter we specifically consider symbol error rate (SER) performance for an M-ary PAM/QAM widely linear (WL) receiver with multiple antennas and multiple co-channel interferers (with mixed PAM/QAM interference type) in a flat Rayleigh fading channel. First, we derive an upper bound (UB) to the SER by averaging the pair-wise error probability (PEP) expression with respect to the pdf of CSI of the desired signal. The resulting SER bound is expressed as a function of the eigenvalues of interference correlation matrix. For the general case of multiple antennas with multiple CCI (with equal average power), we derive two different approximations to this upper bound using the expected value of either the trace or square root of pseudodeterminant of ICM. These two parameters are expressed in closed form using the special properties of real Wishart matrices. We verified the accuracy of the proposed approximations using simulation results. 
Next, using the SER expressions, we showed the trade-off between diversity and IC gain as a function of interference modulation type and the number of antennas. It is shown that both PAM and QAM WL receivers provide a higher diversity order and superior IC gain in the presence of PAM-type interference. More specifically, when interference consists of $M_{1}$ PAM and $M_{2}$ QAM interferers, an $N$ antenna PAM/QAM WL receiver provides full IC with a diversity order: $N-M_{2}-\frac{M_{1}}{2}$. This is significant gain compared to conventional methods that provide full IC gain up to $M$ interferers with a diversity order: $N-M$ for $M<N$.

The rest of the chapter is organized as follows. After introducing the signal model in Section 5.2, the implementation of conventional and WL receivers in ISI free Rayleigh fading channel is discussed in Section 5.3. The SER results for conventional systems is briefly discussed in Section 5.4. The SER analysis for WL receivers is given in Section 5.5 followed by diversity analysis in Section 5.6. We reported simulation results in Section 5.7 followed by a summary of the results of this chapter in Section 5.8.

\subsection{Signal Model}

In this chapter we use a simplified signal model that ignores the side effects such as transmit, receiver filtering and over-sampling. In this chapter we focus our attention to the performance differences caused by the fading channel alone. Therefore, we model the baseband signal at the output of an array of antennas of length $N$ (see Figure 5.1) as:

$$
\mathbf{y}_{k}=\mathbf{h} x_{k}+\mathbf{n}_{k}
$$


where $x_{k}$ denotes the modulation symbols that take complex values when the signal has QAM modulation and $x_{k}$ takes real values when the symbol constellation has PAM,

$$
\mathbf{y}_{k}=\left[y_{k}^{1}, . ., y_{k}^{N}\right]^{\prime}, \quad \mathbf{n}_{k}=\left[n_{k}^{1}, . ., n_{k}^{N}\right]^{\prime}
$$

denote the collection of received signal and noise samples at time instant $k$, arranged in vector format, and

$$
\mathbf{h}=\left[h^{1}, . ., h^{N}\right]^{\prime}
$$

denotes the complex-valued channel coefficients. Assuming that the signals at different antennas have statistically independent fading ${ }^{1}$, each element in $\mathbf{h}$ is modeled as zero mean i.i.d. complex Gaussian variable with variance $C$. We further assume that the modulation symbols are i.i.d. symbols with unit energy. In case of QAM we further assume that the I-Q parts are zero mean uncorrelated symbols with equal variance.

\subsubsection{Interference Model}

The noise component $\mathbf{n}_{k}=\mathbf{w}_{k}+\mathbf{i}_{k}$ is modeled as a sum of a thermal noise term $\mathbf{w}_{k}$ of variance $N_{0}$ per dimension and a sum of $\mathrm{M}$ co-channel interferes

$$
\mathbf{i}_{k}=\sum_{l=1}^{M} \mathbf{g}^{l} x_{k}^{l}
$$

$\mathbf{g}^{l}=\left[g^{l, 1}, . ., g^{l, N}\right]$ ' where $g^{l, n}$ is the impulse response of the $l$ th interferer at $n$th antenna, $x_{k}^{l}$ denotes the modulation alphabets of the $l$ th interfering channel. We assume that average power of all interfering signals is equal to $I_{0}$. Each element $g^{l, n}$ is modeled as an i.i.d. complex Gaussian random variable with zero mean and variance $I_{0}$. To simplify the receiver development, and to simplify the SER analysis,

\footnotetext{
${ }^{1}$ This assumption holds when antennas are spaced several wavelengths apart
} 


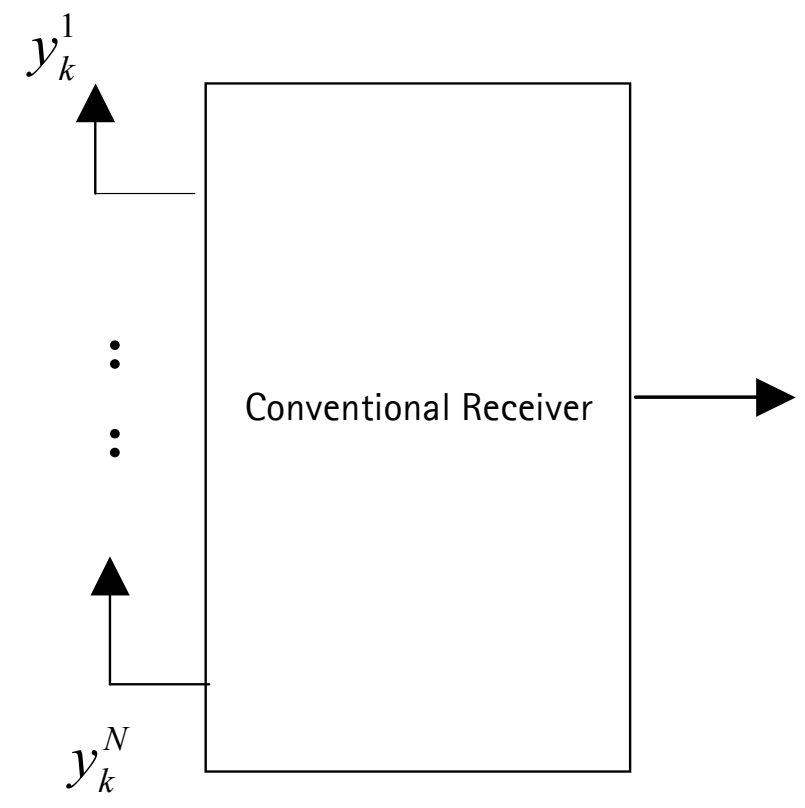

Figure 5.1. Conventional receiver.

we assume that the modulation alphabets $x_{k}^{l}$ are also zero mean, unit variance i.i.d. complex Gaussian random variables. For higher order modulation, the pdf of I/Q parts of $x_{k}^{l}$ can be closely approximated with Gaussian pdf, but in this chapter we use the Gaussian approximation irrespective of modulation size.

\subsection{Receiver Structures}

The MMSE and MMSE-DFE receiver structures developed in the previous chapters for ISI case can be simplified to a great extent when the system becomes ISI free. In the following sections, we briefly revisit the conventional and WL receiver structures for PAM and QAM cases. 


\subsubsection{Conventional Signal Combining}

The receiver structure for a conventional linear (unbiased) MMSE receiver that combines the outputs of antenna branches is shown in Figure 5.1. The filtered signal at the output of an unbiased MMSE detector [5] is given by:

$$
z_{k}=\frac{\mathbf{h}^{\dagger} \mathbf{R}_{n n}^{-1} \mathbf{y}_{k}}{\mathbf{h}^{\dagger} \mathbf{R}_{n n}^{-1} \mathbf{h}}
$$

where

$$
\begin{aligned}
\mathbf{R}_{n n} & \triangleq E\left[\mathbf{n}_{k} \mathbf{n}_{k}^{\dagger}\right] \\
& =E_{x_{k}^{l}}\left[\sum_{l=1}^{M} \mathbf{g}^{l} x_{k}^{l}\left(\sum_{l=1}^{M} \mathbf{g}^{l} x_{k}^{l}\right)^{\dagger}\right]+E\left[\mathbf{w}_{k} \mathbf{w}_{k}^{\dagger}\right] \\
& =\sum_{l=1}^{M} \mathbf{g}^{l} \mathbf{g}^{l \dagger}+N_{0} \mathbf{I}
\end{aligned}
$$

is the correlation matrix of interference plus thermal noise. Further, let

$$
\mathbf{R}_{i i}=\sum_{l=1}^{M} \mathbf{g}^{l} \mathbf{g}^{l \dagger}
$$

denote the interference correlation matrix. The signal to noise ratio (SNR) at the output unbiased MMSE receiver is given by: $\gamma_{c}=\mathbf{h}^{\dagger} \mathbf{R}_{n n}^{-1} \mathbf{h}$. Note that the MMSE detector is functionally equivalent to a maximum likelihood (ML) detector that treats the noise plus interference signal as colored Gaussian noise. Although interference modulation symbols $x_{k}^{l}$ are not Gaussian, we use the Gaussian approximation to simplify the decision metrics and subsequent error rate analysis. With this assumption, the decision metric for a maximum likelihood (ML) detector is given by:

$$
x_{k}=\arg \min _{\hat{x}_{k}} \mathbf{e}_{k}^{\dagger} \mathbf{R}_{n n}^{-1} \mathbf{e}_{k}
$$

where $\mathbf{e}_{k}=\mathbf{y}_{k}-\mathbf{h} \hat{x}_{k}$ and $\hat{x}_{k}$ is a candidate symbol. Both ML and the MMSE receivers provide the same error rate but differ in implementation only. 


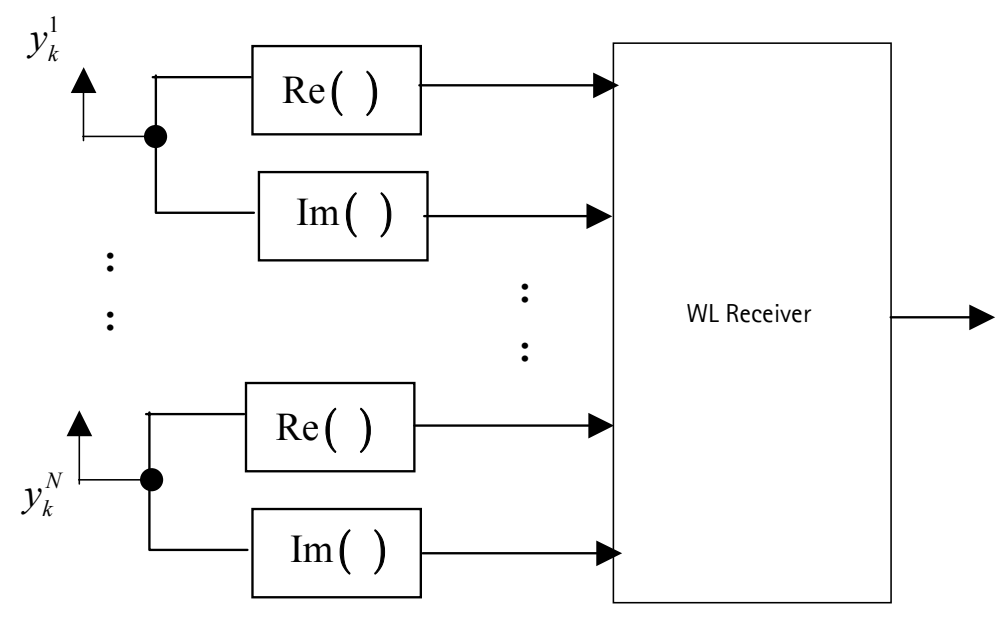

Figure 5.2. WL receiver.

\subsubsection{WL PAM Receiver Implementation in ISI free Channel}

Ignoring the effects of over-sampling, the I-Q signal model can be represented in vector format as:

$$
\tilde{\mathbf{y}}_{k}=\tilde{\mathbf{h}} x_{k}+\tilde{\mathbf{n}}_{k}
$$

where

$$
\begin{aligned}
\tilde{\mathbf{y}}_{k} & =\left[\operatorname{Re}\left(y_{k}^{1}\right), \operatorname{Im}\left(y_{k}^{1}\right), . ., \operatorname{Re}\left(y_{k}^{N}\right), \operatorname{Im}\left(y_{k}^{N}\right)\right]^{\prime} \\
\tilde{\mathbf{h}} & =\left[\operatorname{Re}\left(h^{1}\right), \operatorname{Im}\left(h^{1}\right), . ., \operatorname{Re}\left(h^{N}\right), \operatorname{Im}\left(h^{N}\right)\right]^{\prime} \\
\tilde{\mathbf{n}}_{k} & =\left[\operatorname{Re}\left(n_{k}^{1}\right), \operatorname{Im}\left(n_{k}^{1}\right), . ., \operatorname{Re}\left(n_{k}^{N}\right), \operatorname{Im}\left(n_{k}^{N}\right)\right]^{\prime}
\end{aligned}
$$

denote the real and imaginary parts collected in column vector format. For convenience, we deviated slightly from the I-Q signal notation of Section 2.3. The receiver structure is shown in Figure 5.2. In this case, the receiver applies a vector-valued unbiased MMSE filter whose output is given by

$$
\tilde{z}_{k}=\frac{\tilde{\mathbf{h}}^{\dagger} \mathbf{R}_{\tilde{n} \tilde{n}}^{-1} \tilde{\mathbf{y}}_{k}}{\tilde{\mathbf{h}}^{\dagger} \mathbf{R}_{\tilde{n} \tilde{n}}^{-1} \tilde{\mathbf{h}}}
$$

where

$$
\mathbf{R}_{\tilde{n} \tilde{n}}=\mathrm{E}\left[\tilde{\mathbf{n}}_{k} \tilde{\mathbf{n}}_{k}^{\dagger}\right]
$$


Symbol-by-symbol decisions can be made using the decision variable $\tilde{z}_{k}$ using standard threshold detectors. The SNR at the output of WL MMSE receiver can be obtained as:

$$
\gamma_{\mathrm{WL} \mathrm{PAM}}=\tilde{\mathbf{h}}^{\dagger} \mathbf{R}_{\tilde{n} \tilde{n}}^{-1} \tilde{\mathbf{h}}
$$

Note that the WL receiver can also be implemented alternatively in ML form as discussed in the previous section.

\subsubsection{WL QAM Receiver Implementation in ISI free Channel}

Since QAM symbol has both real and imaginary parts, I-Q split creates a MIMO signal model of type:

$$
\tilde{\mathbf{y}}_{k}=\tilde{\mathbf{X}}_{k} \tilde{\mathbf{h}}+\tilde{\mathbf{n}}_{k}
$$

where $\tilde{\mathbf{X}}_{k} \triangleq \mathbf{I}_{N} \otimes\left[\begin{array}{cc}x_{k, I} & -x_{k, Q} \\ x_{k, Q} & x_{k, I}\end{array}\right]$ denotes $^{2}$ a $2 N \times 2 N$ size modulation matrix and $\otimes$ denotes the matrix Kronecker product [52]. The received signal $\tilde{\mathbf{y}}_{k}$, and noise term $\tilde{\mathbf{n}}_{k}$, channel vector $\tilde{\mathbf{h}}$ are already defined in the previous Section. Note that $\tilde{\mathbf{X}}$ is an orthogonal matrix that satisfies: $\tilde{\mathbf{X}}_{k}^{\dagger} \tilde{\mathbf{X}}_{k}=x_{k, I}^{2}+x_{k, Q}^{2} \mathbf{I}$. We propose that the I-Q parts of the QAM symbols be jointly detected taking into account the correlation between I-Q branches. Because of Gaussian assumption, the pdf of noise plus interference signal can be modeled as:

$$
p\left(\tilde{\mathbf{n}}_{k}\right)=\frac{1}{2 \pi \sqrt{\left|\mathbf{R}_{\tilde{n} \tilde{n}}\right|}} e^{-\left(\tilde{\mathbf{n}}_{k}^{\dagger} \mathbf{R}_{\tilde{n} \tilde{n}}^{-1} \tilde{\mathbf{n}}_{k}\right)} .
$$

Using the above density function, the ML symbol decision metric can be straightforwardly formulated as [53]:

$$
x_{k}=\arg \min _{\hat{x}_{k}} \tilde{\mathbf{e}}_{k}^{\dagger} \mathbf{R}_{\tilde{n} \tilde{n}}^{-1} \tilde{\mathbf{e}}_{k}
$$

\footnotetext{
${ }^{2}$ The symbol $\mathbf{I}_{N}$ denotes an identity matrix of size $N$
} 
where $\tilde{\mathbf{e}}_{k}=\tilde{\mathbf{y}}_{k}-\hat{\mathbf{X}}_{k} \tilde{\mathbf{h}}$. The matrix: $\hat{\mathbf{X}}_{k} \triangleq \mathbf{I}_{N} \otimes\left[\begin{array}{cc}\hat{x}_{k, I} & -\hat{x}_{k, Q} \\ \hat{x}_{k, Q} & \hat{x}_{k, I}\end{array}\right]$ where $\hat{x}_{k}=$ $\hat{x}_{k, I}+j \hat{x}_{k, Q}$ denotes the candidate symbol. The receiver selects the argument that minimizes the minimum distance over all symbol possibilities. Using (5.9), $\tilde{\mathbf{e}}_{k}$ can be expressed as:

$$
\begin{aligned}
\tilde{\mathbf{e}}_{k} & =\tilde{\mathbf{y}}_{k}-\hat{\mathbf{X}}_{k} \tilde{\mathbf{h}} \\
& =\mathbf{E}_{k} \tilde{\mathbf{h}}+\tilde{\mathbf{n}}_{k}
\end{aligned}
$$

where $\mathbf{E}_{k} \triangleq \tilde{\mathbf{X}}_{k}-\hat{\mathbf{X}}_{k}$ denotes the error between the desired and candidate symbol. Because of the MIMO nature of the problem, here, we define the SNR based on the distance metric. Using (5.11), the argument inside the decision metric in (5.10) can be expressed as:

$$
\gamma_{\mathrm{WL} Q \mathrm{QM}}=\tilde{\mathbf{h}}^{\dagger} \mathbf{E}_{k}^{\dagger} \mathbf{R}_{\tilde{n} \tilde{n}}^{-1} \mathbf{E}_{k} \tilde{\mathbf{h}}
$$

Since the ML metric decides in favor of minimum distance, in the above expression, we should substitute the value of $\mathbf{E}_{k}$ that corresponds to the minimum distance error event to obtain the SNR of WL receiver.

\subsubsection{Structure of I-Q Noise plus Interference Correlation Matrix}

Since CCI is allowed to have either PAM or QAM constellation we model the interference signal as a sum of $M_{1}$ PAM interferers and $M_{2}$ QAM interferers. The noise term $\tilde{\mathbf{n}}_{k}$ can be explicitly represented in terms of individual interference terms as:

$$
\tilde{\mathbf{n}}_{k}=\sum_{l=1}^{M_{1}} \tilde{\mathbf{g}}^{l} x_{k}^{l}+\sum_{l=1}^{M_{2}} \tilde{\mathbf{G}}^{l} \tilde{\mathbf{x}}_{k}^{l}+\tilde{\mathbf{w}}_{k}
$$

where

$$
\tilde{\mathbf{g}}^{l}=\left[\operatorname{Re}\left(g^{l, 1}\right), \operatorname{Im}\left(g^{l, 1}\right), . ., \operatorname{Re}\left(g^{l, N}\right), \operatorname{Im}\left(g^{l, N}\right)\right]^{\prime}
$$


denote the individual I-Q parts of the $l$ th PAM channel coefficients, $x_{k}^{l}$ are PAM modulation symbols,

$$
\tilde{\mathbf{G}}^{l}=\left[\mathbf{G}^{l, 1}, . ., \mathbf{G}^{l, N}\right]^{\prime}
$$

where

$$
\mathbf{G}^{l, q} \triangleq\left(\begin{array}{cc}
\operatorname{Re}\left(g^{l, q}\right) & \operatorname{Im}\left(g^{l, q}\right) \\
-\operatorname{Im}\left(g^{l, q}\right) & \operatorname{Re}\left(g^{l, q}\right)
\end{array}\right)
$$

$q$ being index, denotes the I-Q channel matrix for $l$ th QAM interferer,

$$
\tilde{\mathbf{x}}_{k}^{l}=\left[\operatorname{Re}\left(x_{k}^{l}\right), \operatorname{Im}\left(x_{k}^{l}\right)\right]^{\prime}
$$

represents the I-Q parts of QAM interfering modulation symbols and

$$
\tilde{\mathbf{w}}_{k}=\left[\operatorname{Re}\left(w_{k}^{1}\right), \operatorname{Im}\left(w_{k}^{1}\right), . ., \operatorname{Re}\left(w_{k}^{N}\right), \operatorname{Im}\left(w_{k}^{N}\right)\right]^{\prime}
$$

represents the I-Q parts of thermal noise component with variance $\frac{N_{0}}{2}$ per dimension. Then the noise plus interference correlation matrix can be expressed as:

$$
\mathbf{R}_{\tilde{n} \tilde{n}}=\mathbf{R}_{\tilde{i} \tilde{i}}+\frac{N_{0}}{2} \mathbf{I}
$$

where $\mathbf{R}_{\tilde{i} \tilde{i}}$ denotes the ICM:

$$
\mathbf{R}_{\tilde{i} \tilde{i}}=\sum_{l=1}^{M_{1}} \tilde{\mathbf{g}}^{l} \tilde{\mathbf{g}}^{l \dagger}+\frac{1}{2} \sum_{l=1}^{M_{2}} \tilde{\mathbf{G}}^{l} \tilde{\mathbf{G}}^{l \dagger} .
$$

\subsection{SER for Conventional Receivers}

In [47], the SER for an M-PSK conventional receiver introduced in Section (5.3.1) was obtained in Eq (15) of [47] as:

$$
P_{c}=\frac{1}{\pi} E_{\bar{\lambda}}\left[\int_{0}^{\Theta} A(\theta) \prod_{i=1}^{N_{\min }}\left[\frac{\sin ^{2}(\theta)}{\sin ^{2}(\theta)+c_{\mathrm{MPSK}} \frac{C}{\lambda_{i}}+N_{0}}\right] d \theta\right]
$$

where $\bar{\lambda}_{i}, i=1, . ., N_{\text {min }}$ denotes the eigenvalues of $\mathbf{R}_{n n}, N_{\min }$ denotes its rank given by:

$$
N_{\min }=\min (N, M), \quad c_{\mathrm{MPSK}}=\sin ^{2} \frac{\pi}{M}, \quad \Theta=\frac{\pi(M-1)}{M},
$$




$$
A(\theta) \triangleq\left[\frac{\sin ^{2}(\theta)}{\sin ^{2}(\theta)+c_{\mathrm{MPSK}} \frac{C}{N_{0}}}\right]^{N-N_{\min }}
$$

and $E_{\bar{\lambda}}$ denotes the expectation with respect to the joint pdf of eigenvalues $\bar{\lambda}_{i}$. Because of our starting assumption that the multiple interferers have independent Rayleigh fading with equal average power, it can be shown that the interference correlation matrix (given by (5.4)) $\mathbf{R}_{i i}=\sum_{l=1}^{M} \mathbf{g}^{l} \mathbf{g}^{l \dagger}$ has complex Wishart distribution. The joint pdf of eigenvalues of $\mathbf{R}_{i i}$ is given in Eq (12) of [47]. The expectation operation in (5.15) requires an $N_{\text {min }}$ fold integration with respect to the pdf of each of the eigenvalues $\lambda_{i}$ that does not have a closed form solution for arbitrary values of $N$ and M. In [47], Chaini et al. obtained several bounds and approximations to simplify the SER calculations. In particular, in Section V-B, they derived an useful bound based on the expected value of trace of $\mathbf{R}_{n n}$ as:

$$
P_{c, B}=\frac{1}{\pi} \int_{0}^{\Theta} A(\theta)\left[\frac{\sin ^{2}(\theta)}{\sin ^{2}(\theta)+c_{\mathrm{MPSK}} \frac{C}{I_{0} N_{\max }+N_{0}}}\right]^{N_{\min }} d \theta
$$

where $N_{\max }=\max (N, M)$.

\subsubsection{Discussion}

In the limiting case as $N_{0} \rightarrow 0, A(\theta) \rightarrow\left[\frac{\sin ^{2}(\theta)}{c_{\text {MPSK }} \frac{C}{N_{0}}}\right]^{N-N_{\min }}$. Hence the diversity order for this system, defined as the exponent of $\frac{C}{N_{0}}$, is given by $d_{c o n v}=N-N_{\min }$. Recall that, from (5.3), the ICM is given by: $\mathbf{R}_{i i}=\sum_{l=1}^{M} \mathbf{g}^{l} \mathbf{g}^{l \dagger}$. Since $\mathbf{g}^{l}$ is a column vector of length $N$, the rank of $\mathbf{R}_{i i}$ can be expressed as: $N_{\min }=\min (N, M)$. When the number of interferers $M$ is less than the total number of antennas $N, \mathbf{R}_{i i}$ becomes rank deficient i.e. $N_{\min }=M$. In that case, the diversity order is given by: $d_{c o n v}=N-M$. Also, as $N_{0} \rightarrow 0$, the SER becomes zero, irrespective of interference power. Therefore, we say that the conventional receiver fully rejects $M$ interferers with a diversity order: $N-M$ as long as $M<N$. In the opposite case when $\mathbf{R}_{i i}$ becomes full rank i.e. when 
$M \geq N$, the receiver can not fully suppress interference, but gives full diversity. In this case the diversity order can only be measured with respect to the exponent of signal to noise plus interference ratio (SINR) term $\frac{C}{N_{\max } I_{0}+N_{0}}$ in $(5.15)$.

\subsection{SER Analysis for WL Receivers}

The conventional SER results of previous section are specific to M-PSK systems with conventional signal combining. In this section, we consider a general upper bound to the SER for M-ary PAM and QAM systems with WL processing. In order to derive this bound we use the standard pair wise error probability formulation that is similar to the commonly used union bound. In the following section we first formulate the PEP expressions for PAM and QAM signaling schemes with WL processing. In Section 5.5.3, we derive closed form expressions for the SER using certain bounds and approximations.

\subsubsection{PEP for WL PAM Receiver}

The pair wise error probability (PEP) defined as the probability of transmitting a correct symbol $x_{k}$ and erroneously receiving $\hat{x}_{k}$ can be formulated as [53]:

$$
P\left(x_{k} \rightarrow \hat{x}_{k}\right)=Q\left[\sqrt{d_{k}^{2} \gamma_{\mathrm{WL} \text { PAM }}}\right]
$$

where $d_{k}^{2}=\frac{\left|x_{k}-\hat{x}_{k}\right|^{2}}{4}$ denotes the (normalized) squared Euclidean distance and

$$
Q(x) \triangleq \frac{1}{\sqrt{2 \pi}} \int_{x}^{\infty} e^{-\frac{a^{2}}{2}} d a
$$

and $\gamma_{\mathrm{WL} \text { PAM }}=\tilde{\mathbf{h}}^{\dagger} \mathbf{R}_{\tilde{n} \tilde{n}}^{-1} \tilde{\mathbf{h}}$ which is given by (5.8). Since the channel and interfering vectors are random quantities, the average PEP can be obtained as:

$$
P_{\mathrm{PAM}}\left(x_{k} \rightarrow \hat{x}_{k}\right)=E\left[Q\left(\sqrt{d_{k}^{2} \tilde{\mathbf{h}}^{\dagger} \mathbf{R}_{\tilde{n} \tilde{n}}^{-1} \tilde{\mathbf{h}}}\right)\right]
$$

where $E$ denotes the expectation with respect to the pdf of $\tilde{\mathbf{h}}^{\dagger} \mathbf{R}_{\tilde{n} \tilde{n}}^{-1} \tilde{\mathbf{h}}$. 


\subsubsection{PEP for WL QAM Receiver}

Using the signal model of Section 5.3.3, the PEP for QAM can be generalized as:

$$
P_{\mathrm{QAM}}\left(x_{k} \rightarrow \hat{x}_{k}\right)=E\left[Q\left(\sqrt{\frac{\gamma_{\mathrm{WL} \mathrm{QAM}}}{4}}\right)\right]
$$

where $\gamma_{\mathrm{WL} \text { QAM }}=\tilde{\mathbf{h}}^{\dagger} \mathbf{E}_{k}^{\dagger} \mathbf{R}_{\tilde{n} \tilde{n}}^{-1} \mathbf{E}_{k} \tilde{\mathbf{h}}$. The matrix $\mathbf{E}_{k}=\tilde{\mathbf{X}}_{k}-\hat{\mathbf{X}}_{k}$ is an orthogonal matrix because $\tilde{\mathbf{X}}_{k}, \hat{\mathbf{X}}_{k}$ are orthogonal matrices by definition. Therefore, $\mathbf{E}_{k}^{\dagger} \mathbf{E}_{k}$ can be expressed as:

$$
\mathbf{E}_{k}^{\dagger} \mathbf{E}_{k}=\left(e_{k, I}^{2}+e_{k, Q}^{2}\right) \mathbf{I}=\left|x_{k}-\hat{x}_{k}\right|^{2} \mathbf{I}
$$

where $e_{k, I} \triangleq x_{k, I}-\hat{x}_{k, I}$ and $e_{k, Q} \triangleq x_{k, Q}-\hat{x}_{k, Q}$. Now we rewrite the numerator of the argument inside the Q-function as:

$$
\tilde{\mathbf{h}}^{\dagger} \mathbf{E}_{k}^{\dagger} \mathbf{R}_{\tilde{n} \tilde{n}}^{-1} \mathbf{E}_{k} \tilde{\mathbf{h}}=\left(e_{k, I}^{2}+e_{k, Q}^{2}\right) \tilde{\mathbf{h}}^{\dagger} \overline{\mathbf{E}}^{\dagger}{ }_{k} \mathbf{R}_{\tilde{n} \tilde{n}}^{-1} \overline{\mathbf{E}}_{k} \tilde{\mathbf{h}}
$$

where $\overline{\mathbf{E}}_{k} \triangleq \frac{\mathbf{E}_{k}}{\sqrt{\left(e_{k, I}^{2}+e_{k, Q}^{2}\right)}}$ becomes a unitary matrix after normalization. Let

$$
\overline{\mathbf{h}}=\overline{\mathbf{E}}_{k} \tilde{\mathbf{h}} .
$$

Since the individual elements of the complex channel $\mathbf{h}$ are assumed to be i.i.d. complex Gaussian, and since the I-Q parts of a complex Gaussian process are i.i.d. random variables, $\tilde{\mathbf{h}}$ becomes: $N\left(0, \frac{C}{2} \mathbf{I}_{2 N}\right)$. Further, we can show that the projection of a zero mean i.i.d. Gaussian vector $\tilde{\mathbf{h}}$ onto an unitary matrix $\overline{\mathbf{E}}_{k}$ yields another zero mean i.i.d. Gaussian vector $\overline{\mathbf{h}}=\left[\bar{h}_{1}, . ., \bar{h}_{2 N}\right]$ with the same pdf as $\tilde{\mathbf{h}}$. Using this result, we can express (5.21) in terms of $\overline{\mathbf{h}}$ as

$$
\left(e_{k, I}^{2}+e_{k, Q}^{2}\right) \tilde{\mathbf{h}}^{\dagger} \overline{\mathbf{E}}_{k}^{\dagger} \mathbf{R}_{\tilde{n} \tilde{n}}^{-1} \overline{\mathbf{E}}_{k} \tilde{\mathbf{h}}=\left(e_{k, I}^{2}+e_{k, Q}^{2}\right) \overline{\mathbf{h}}^{\dagger} \mathbf{R}_{\tilde{n} \tilde{n}}^{-1} \overline{\mathbf{h}}
$$

Using (5.18),(5.19),(5.22), using the fact that $\tilde{\mathbf{h}}$ and $\overline{\mathbf{h}}$ have same pdf, the PEP expressions for PAM and QAM cases take identical forms with the exception of constellation 
specific scaling factors $d_{k}^{2}$. Hence the PEP for either PAM or QAM signaling can be represented in universal form as:

$$
\mathrm{PEP}=E\left[Q\left(\sqrt{d_{k}^{2} \overline{\mathbf{h}}^{\dagger} \mathbf{R}_{\tilde{n} \tilde{n}}^{-1} \overline{\mathbf{h}}}\right)\right], \quad d_{k}^{2}=\frac{\left|x_{k}-\hat{x}_{k}\right|^{2}}{4} .
$$

Therefore we can say that the interference suppression capability of a WL receiver is completely independent of the modulation type used by the desired signal (i.e. whether PAM or QAM is used) but the gain is decided mainly by the noise plus interference correlation matrix structure.

\subsubsection{Error Rate Analysis}

If we assume that there are $K_{d}$ error events that contribute to the same minimum distance $d_{m i n}$, using the PEP expression (5.23), the symbol error rate for PAM/QAM modulation can be upper bounded as [53]:

$$
P \leq K_{d} E\left[Q\left(\sqrt{d_{m i n}^{2} \overline{\mathbf{h}}^{\dagger} \mathbf{R}_{\tilde{n} \tilde{n}}^{-1} \overline{\mathbf{h}}}\right)\right]
$$

Note that the above upper bound is exact for BPSK, and provides accurate results for higher order modulation for low error rates regions only. The matrix $\mathbf{R}_{\tilde{n} \tilde{n}}^{-1}$ can be expressed as:

$$
\mathbf{R}_{\tilde{n} \tilde{n}}^{-1}=\mathbf{U}^{\dagger} \Lambda \mathbf{U}
$$

where $\mathbf{U}$ is an orthogonal matrix and $\Lambda=\operatorname{diag}\left[\frac{1}{\lambda_{1}}, \ldots, \frac{1}{\lambda_{2 N}}\right]$. Note that $\lambda_{k}$ represents the eigenvalues of $\mathbf{R}_{\tilde{n} \tilde{n}}$. Using ( 5.25), we express the argument inside the Q-function as

$$
d_{m i n}^{2} \overline{\mathbf{h}}^{\dagger} \mathbf{R}_{\tilde{n} \tilde{n}}^{-1} \overline{\mathbf{h}}^{\dagger}=d_{\min }^{2} \omega^{\dagger} \Lambda \omega=d_{\min }^{2} \sum_{l=1}^{2 N} \frac{\omega_{l}^{2}}{\lambda_{l}}
$$

where

$$
\omega=\left[\omega_{1}, . ., \omega_{2 N}\right]=\mathbf{U} \overline{\mathbf{h}}
$$


is also zero mean i.i.d Gaussian i.e. $\omega_{l}$ are: $N\left(0, \frac{C}{2}\right)$. Using this result (5.24) can be expressed as:

$$
P \leq K_{d} E\left[Q\left(\sqrt{d_{\min }^{2} \sum_{l=1}^{2 N} \frac{\omega_{l}^{2}}{\lambda_{l}}}\right)\right] .
$$

To simplify the analysis we further we upper bound the Q-function as a sum of exponentials as [54]:

$$
Q(x)<\frac{1}{12} e^{\frac{-x^{2}}{2}}+\frac{1}{4} e^{\frac{-2 x^{2}}{3}}
$$

This approximation allows a closed form evaluation of expectation with respect to $\omega_{l}$ while the error caused by the approximation is negligibly small for the SER of practical interest. Using (5.28) and using the fact that $\omega_{l}$ are statistically independent real Gaussian random variables for different $l$, we can further upper bound (5.27) as:

$$
P \leq \frac{K_{d}}{12} \prod_{l=1}^{2 N} E_{\left(\omega_{l}, \lambda_{l}\right)}\left(e^{d_{\min }^{2} \frac{\omega_{l}^{2}}{2 \lambda_{l}}}\right)+\frac{K_{d}}{4} \prod_{l=1}^{2 N} E_{\left(\omega_{l}, \lambda_{l}\right)}\left(e^{2 d_{\min }^{2} \frac{\omega_{l}^{2}}{3 \lambda_{l}}}\right)
$$

Next we use the following result. When $u=N\left(0, \sigma^{2}\right), E\left[e^{\alpha u^{2}}\right]=\frac{1}{\sqrt{\left(1+2 \alpha \sigma^{2}\right)}}$. Using this result we first evaluate the expectation with respect to $\omega_{l}$ in $(5.29)$ to get

$$
\begin{aligned}
P & \leq E_{\lambda_{l}}\left[\frac{K_{d}}{12 \prod_{l=1}^{2 N} \sqrt{\left(1+\frac{d_{\min }^{2} C}{2 \lambda_{l}}\right)}}+\frac{K_{d}}{4 \prod_{l=1}^{2 N} \sqrt{\left(1+\frac{2 d_{\min }^{2} C}{3 \lambda_{l}}\right)}}\right] \\
& =E_{\lambda_{l}}\left[\sum_{i=1,2}\left(\frac{K_{i}}{\prod_{l=1}^{2 N} \sqrt{\left(1+\frac{c_{i} C}{\lambda_{l}}\right)}}\right)\right]
\end{aligned}
$$

where the constants $c_{1}=\frac{d_{\min }^{2}}{2}, c_{2}=\frac{2 d_{\min }^{2}}{3}, K_{1}=\frac{K_{d}}{12}, K_{2}=\frac{K_{d}}{4}$ are introduced for compactness. Let $m$ denote the rank of $2 N \times 2 N$ matrix $\mathbf{R}_{\tilde{i} \tilde{i}}$. Then $\mathbf{R}_{\tilde{i} \tilde{i}}$ has exactly $m$ non-zero eigenvalues denoted as: $\tilde{\lambda}_{l}, l=1, . ., m$ and the remaining $2 N-m$ eigenvalues are zero. Using this, the eigenvalues of $\mathbf{R}_{\tilde{n} \tilde{n}}$ can be expressed as:

$$
\begin{aligned}
\lambda_{l} & =\tilde{\lambda}_{l}+\frac{N_{0}}{2}, \text { for } l=1, . ., m \\
& =0, \text { for } l=m+1, . ., 2 N .
\end{aligned}
$$


Using this result we can rearrange the terms in (5.30) as

$$
P \leq \sum_{i=1,2}\left[\frac{K_{i}}{\left(1+\frac{2 c_{i} C}{N_{0}}\right)^{N-\frac{m}{2}}} E_{\tilde{\lambda}_{l}}\left(\frac{1}{\prod_{l=1}^{m} \sqrt{\left(1+\frac{c_{i} C}{\tilde{\lambda}_{l}+\frac{N_{0}}{2}}\right)}}\right)\right]
$$

In the above expression we still need to calculate the expectation with respect to $\tilde{\lambda}_{l}$. This aspect is addressed in following sections where we obtained two useful approximations that will be used to express (5.33) in closed form.

\subsubsection{SER Approximations}

The SER upper bound given by (5.33) requires an expectation operation:

$$
E_{\tilde{\lambda}_{l}}\left[\frac{1}{\prod_{l=1}^{m} \sqrt{\left(1+\frac{c_{i} C}{\tilde{\lambda}_{l}+\frac{N_{0}}{2}}\right)}}\right]
$$

First we consider a system with $N$ antennas and $M$ PAM interferers of equal average power. The extension to mixed interference case is given in Section 5.5.4.3. With $M$ PAM interferers, the interference correlation matrix takes the form:

$$
\mathbf{R}_{\tilde{i} \tilde{i}}=\sum_{l=1}^{M} \tilde{\mathbf{g}}^{l} \tilde{\mathbf{g}}^{l \dagger} .
$$

Since $\tilde{\mathbf{g}}$ is an i.i.d. real gaussian vector, $\mathbf{R}_{\tilde{n} \tilde{n}}$ belongs to the class of (real) Wishart matrices whose pdf is given in [47]. Therefore evaluation of expectation operation in (5.34) requires integration over the joint pdf of eigenvalues $\mathbf{R}_{\tilde{i} i}$. In this case, it is difficult to obtain a closed form result. Numerical integration has to be used. We avoid this approach, instead, we provide simple bounds and approximations to (5.34) using the special properties of real Wishart matrices (that are discussed in Appendix E.1). First, we derive an approximation to (5.34) using the expected value of square 
root of pseudo-determinant of $\mathbf{R}_{\tilde{i}}$. In Section 5.5.4.2, a simple approximation is derived based on the trace of $\mathbf{R}_{\tilde{i}}$.

\subsubsection{Approximation-1}

Let us consider an interference limited system where $N_{0}<<I_{0}<C_{0}$. In that case,

the term $\frac{c_{i} C}{\tilde{\lambda}_{l}+\frac{N_{0}}{2}}>>1$ for most channel realizations. Hence we approximate the denominator terms of (5.34) (when $N_{0} \rightarrow 0$ ) as:

$$
\prod_{l=1}^{m} \sqrt{\left(1+\frac{c_{i} C}{\tilde{\lambda}_{l}+\frac{N_{0}}{2}}\right)} \sim \prod_{l=1}^{m} \sqrt{\frac{c_{i} C}{\tilde{\lambda}_{l}}}
$$

Using this result, we can approximate (5.33) as:

$$
P \leq \sum_{i=1,2}\left[\frac{K_{i}}{\left(1+\frac{2 c_{i} C}{N_{0}}\right)^{N-\frac{m}{2}}} E_{\tilde{\lambda}_{l}}\left(\prod_{l=1}^{m} \sqrt{\frac{\tilde{\lambda}_{l}}{c_{i} C}}\right)\right]
$$

Using Theorem E.1.4 from Appendix E.1 the expected value of square root of non-zero eigenvalues (pseudo-determinant) of $\mathbf{R}_{\tilde{i} \tilde{i}}$ can be expressed as:

$$
E\left[\prod_{l=1}^{m} \sqrt{\tilde{\lambda}_{l}}\right]=\left(\frac{I_{0}}{2}\right)^{\frac{m}{2}} \prod_{i=1}^{m} \frac{\Gamma\left(\frac{n-i+1}{2}+\frac{1}{2}\right)}{\Gamma\left(\frac{n-i+1}{2}\right)}
$$

where $m=\min (2 N, M), n=\max (2 N, M)$. Substituting (5.36) in (5.35) we get:

$$
P_{1}=\rho \sum_{i=1,2}\left[\frac{K_{i}}{\left(1+\frac{2 c_{i} C}{N_{0}}\right)^{\frac{2 N-m}{2}}\left(\frac{2 c_{i} C}{I_{0}}\right)^{\frac{m}{2}}}\right]
$$

where $\rho \triangleq \prod_{i=1}^{m} \frac{\Gamma\left(\frac{n-i+1}{2}+\frac{1}{2}\right)}{\Gamma\left(\frac{n-i+1}{2}\right)}$.

\subsubsection{Approximation-2}

The SER approximation developed in the previous section is a tight approximation only when $N_{0}<<I_{0}<C_{0}$. In this section we remove this restriction and present a 
SER approximation that works for other cases as well. In (5.33) the SER is expressed as

$$
P \leq \sum_{i=1,2}\left[\frac{K_{i}}{\left(1+\frac{2 c_{i} C}{N_{0}}\right)^{\left(N-\frac{m}{2}\right)}} E_{\tilde{\lambda}_{l}}\left(\frac{1}{\prod_{l=1}^{m} \sqrt{\left(1+\frac{c_{i} C}{\tilde{\lambda}_{l}+\frac{N_{0}}{2}}\right)}}\right)\right]
$$

Since the eigenvalues $\tilde{\lambda}_{l}$ are positive, the above SER expression is concave in each individual eigenvalue when others are fixed. But the function is neither globally convex nor concave. Yet, we apply Jensen's inequality as suggested by Chaini et.al. in [47] to approximate $(5.38)$ as:

$$
P_{2}=\sum_{i=1,2}\left[K_{i} \frac{1}{\left(1+\frac{2 c_{i} C}{N_{0}}\right)^{\frac{2 N-m}{2}} \sqrt{\prod_{l=1}^{m}\left(1+\frac{c_{i} C}{E\left[\tilde{\lambda}_{l}+\frac{N_{0}}{2}\right]}\right)}}\right] .
$$

Numerical evaluation of (5.39) requires one to calculate the expected values of $\tilde{\lambda}_{l}$. The joint pdf of eigenvalues $\tilde{\lambda}_{l}$ given by Theorem E.1.1 can be used to calculate these expected values for a few special cases, (closed for evaluation is tractable for $M=2$, arbitrary $N$ or $N=1$, arbitrary $M$ ) but closed form evaluation for the general case is difficult. To overcome this problem we use the following inequality from Section V-B of [47]: $\prod_{i=1}^{m} \frac{A}{A+\frac{B}{x_{i}}} \leq\left[\frac{A}{A+\frac{B m}{\sum_{i=1}^{p} x_{i}}}\right]^{m}$ to further upper bound the second part of (5.39) as:

$$
\left[\frac{1}{\sqrt{\prod_{l=1}^{m}\left(1+\frac{c_{i} C}{E\left[\tilde{\lambda}_{l}+\frac{N_{0}}{2}\right]}\right)}}\right]<\frac{1}{\left(1+\frac{c_{i} C m}{m \frac{N_{0}}{2}+\sum_{l=1}^{m} E\left[\tilde{\lambda}_{l}\right]}\right)^{\frac{m}{2}}}
$$


Note that $E\left[\sum_{l=1}^{m} \tilde{\lambda}_{l}\right]=E\left[\operatorname{Tr}\left(\mathbf{R}_{\tilde{n} \tilde{n}}\right)\right]=m n \frac{I_{0}}{2}$. Substituting this result in (5.40), we get:

$$
P_{2} \leq \sum_{i=1,2}\left[K_{i} \frac{1}{\left(1+\frac{2 c_{i} C}{N_{0}}\right)^{\frac{2 N-m}{2}}\left(1+\frac{c_{i} C}{\frac{N_{0}}{2}+n \frac{I_{0}}{2}}\right)^{\frac{m}{2}}}\right]
$$

\subsubsection{Mixed Interference Scenario}

When interference has a mixture of PAM and QAM interferers $\mathbf{R}_{\tilde{n} \tilde{n}}$ is no longer a Wishart matrix. Hence the SER approximation given by Approximation-1 can not applied directly. However, we can simplify the analysis and obtain useful approximation to the SER by replacing each QAM interferer with two PAM interferers (This approximation was justified in Chapter 4). In that case approximation-1 given by (5.41) can be used directly with the following parameters:

$$
\begin{aligned}
m & =\min \left(2 N, M_{1}+2 M_{2}\right) \\
n & =\max \left(2 N, M_{1}+2 M_{2}\right) .
\end{aligned}
$$

Note that the above expressions can be obtained straightforwardly by using the results from the proof of Theorem E.1.4. Also, since the trace of $\mathbf{R}_{\tilde{i} \tilde{i}}$ is independent of modulation type employed by the individual interferer, the trace based approximation-2 applies to mixed interference case as well. In the following section we use the SER bounds to analyze the diversity and IC properties of WL receivers.

\subsection{Diversity-IC Trade-off}

In Section 5.5.4.1 the SER for interference limited conditions is upper bounded as:

$$
P_{1}=\rho \sum_{i=1,2}\left[\frac{K_{i}}{\left(1+\frac{2 c_{i} C}{N_{0}}\right)^{\frac{2 N-m}{2}}\left(\frac{2 c_{i} C}{I_{0}}\right)^{\frac{m}{2}}}\right] .
$$


We use the above result to describe the diversity-IC trade-off for a system with $N$ antennas, $M_{1}$ PAM and $M_{2}$ QAM interferers. Let us consider the SER behavior when $\mathbf{R}_{\tilde{i} \tilde{i}}$ is rank deficient i.e. when $m<2 N$. In the asymptotic case as $N_{0} \rightarrow 0$, we say that the WL receiver provides a diversity order: $d_{W L}=N-\frac{m}{2}$ which is given by exponent of the SNR term $\frac{C}{N_{0}}$ in (5.43). Recall that in mixed interference case, the rank of $\mathbf{R}_{\tilde{i} \tilde{i}}$ is calculated in (5.42) as: $m=\min \left(2 N, M_{1}+2 M_{2}\right)$. When $m<2 N$, the rank becomes: $m=M_{1}+2 M_{2}$. In that case, the diversity order is given by

$$
d_{W L}=N-M_{2}-\frac{M_{1}}{2}
$$

Therefore, at high SNR, the SER is dictated by thermal noise level rather than interference power level ${ }^{3}$. In fact, from the SER expression (5.43) we can see that receiver provides a zero error rate as $N_{0} \rightarrow 0$, irrespective of interference power level. Hence, we say that the WL receiver fully eliminates $M_{1}$ PAM and $M_{2}$ QAM interferers when $M_{1}+2 M_{2}<2 N$ with a diversity benefit: $d_{W L}=N-M_{2}-\frac{M_{1}}{2}$.

Next, we consider the differences in the diversity performance of conventional and WL receivers. Let $M_{1}+M_{2}=M$. In Section 5.5 we have shown that the diversity order of a conventional receiver is given by

$$
d_{C o n v}=N-M \quad \text { when } \quad M<N \text {. }
$$

Using (5.44), (5.45) we can show that:

$$
d_{W L}-d_{C o n v}=\frac{M_{1}}{2}
$$

\footnotetext{
${ }^{3}$ When $\mathbf{R}_{\tilde{i} \tilde{i}}$ has full rank $m=2 N$ i.e. when $M_{1}+2 M_{2} \geq 2 N$, the SER is limited by the interference power rather than thermal noise level. In this type of system, one can define diversity order (i.e. the slope of SER) with respect to SIR (signal to interference power ratio) only. In this case the WL receiver provides full diversity $N$ (given by exponent of $\frac{C}{I_{0}}$ in (5.37), but gives rather limited IC gain. In this case, the multiplicative factor $\rho$ in (5.37) represents the SER reduction compared to a conventional system.
} 
Table 5.1. Diversity-IC trade-off for WL receivers with $N=2$

\begin{tabular}{|c|c|}
\hline$d_{W L}=N-M_{2}-\frac{M_{1}}{2}$ & $\left(M_{1}, M_{2}\right), M_{1}+2 M_{2}<2 N$ \\
\hline 0.5 & $(3,0),(1,1)$ \\
\hline 1 & $(2,0),(0,1)$ \\
\hline 1.5 & $(1,0)$ \\
\hline
\end{tabular}

Table 5.2. Diversity-IC trade-off for WL receivers with $N=4$

\begin{tabular}{|c|c|}
\hline$d_{W L}=N-M_{2}-\frac{M_{1}}{2}$ & $\left(M_{1}, M_{2}\right), M_{1}+2 M_{2}<2 N$ \\
\hline 0.5 & $(7,0),(5,1),(3,2),(1,3)$ \\
\hline 1 & $(6,0),(4,1),(2,2),(0,3)$ \\
\hline 1.5 & $(5,0),(3,1),(1,2)$ \\
\hline 2 & $(4,0),(2,1),(0,2)$ \\
\hline 2.5 & $(3,0),(1,1)$ \\
\hline 3 & $(2,0),(0,1)$ \\
\hline 3.5 & $(1,0)$ \\
\hline
\end{tabular}

The above result indicates that, when the number of interferers to be suppressed is kept the same for conventional and WL cases, the diversity gain of WL receiver increases with an increase in the number of PAM interferers. Therefore, from diversity and IC point of view, it is beneficial to operate the system with more PAM components than QAM.

Table 5.3. Diversity-IC trade-off for conventional receivers with $N=4$

\begin{tabular}{|c|c|}
\hline$d_{\text {Conv }}=N-M$ & $M, M<N$ \\
\hline 1 & 3 \\
\hline 2 & 2 \\
\hline 3 & 1 \\
\hline
\end{tabular}




\subsubsection{Discussion}

Using the PEP upper bound we first showed that the IC properties of the WL receivers do not depend on the modulation type of wanted signal (i.e PAM/QAM) but depends mainly on the rank of ICM. From IC point of view, we have also shown that each QAM signal can be treated as two equivalent PAM signals. This view provides a simple guideline to the system designer to optimize the system with a mixture of PAM and QAM signals.

Next, we established the trade-off between diversity and IC gain for both conventional and WL receivers. In Table 5.2, 5.1 we tabulated the diversity order versus the number of PAM/QAM interferers that the WL receiver can suppress for $N=4$ and $N=2$ respectively. In the second column of these tables, we plotted all possible combinations $\left(M_{1}, M_{2}\right)$ that gives a given diversity order $d_{W L}$ satisfying the constraint: $M_{1}+2 M_{2}<2 N$. For example, single antenna WL receiver can suppress single PAM interference whereas a two antenna WL receiver can suppress a maximum of 3-PAM interferers. In a mixed interferer scenario, with 2-antennas, the WL receiver can reject a total of two interferers (a single PAM plus a single QAM) whereas the conventional receiver can reject a single interferer only. Clearly, the WL receivers provide an advantage when interference consists of PAM or a mixture of PAM and QAM interferers. Also note that when the total number of interferers to be canceled is fixed, WL receivers provide a higher diversity order than conventional receivers. As an example, consider a 4-antenna system with 3-PAM interferers. In this the conventional receiver provides a diversity of order equal to 1 whereas the WL-receiver gives a diversity order 2.5. Clearly, WL receivers provide very good diversity-IC trade-off with a fewer number of antennas than would be necessary for a conventional systems. Hence, we can say that, WL receivers are not only useful for enhancing 


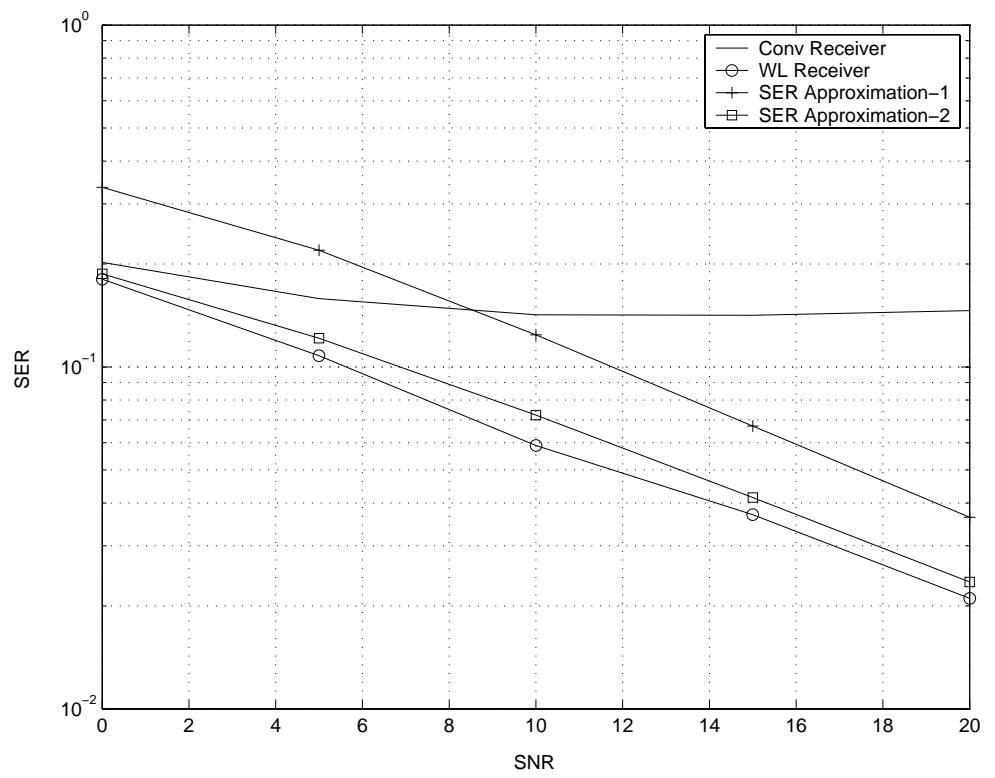

Figure 5.3. Single Antenna: BPSK desired-single BPSK CCI $\left(C=I_{0}=1\right)$.

performance but also offer a low complexity solution to the CCI mitigation problem.

\subsection{Simulation Results}

We provide simulation results for a system that uses a mixture of BPSK and QPSK modulation alphabets. We show results for signal and dual antenna cases with multiple interferers. The simulated results are compared with the bounds and approximations for WL receiver case only. For comparison the simulated SER results for conventional receivers are also shown. The SER results are reported in two formats. In one case the error rate is plotted as function of $\mathrm{C} / \mathrm{I}$ (signal to total interference power ratio) while fixing the $\frac{I_{0}}{N_{0}}$ level at $20 \mathrm{~dB}$. In the second case we set $C=I_{0}=1$ and plot the error rate as a function of SNR defined as $\frac{C}{N_{0}}$.

Figure 5.3 shows SER for BPSK in the presence of a single BPSK CCI where the SER is plotted as a function of SNR. In this case the SER for conventional receiver 


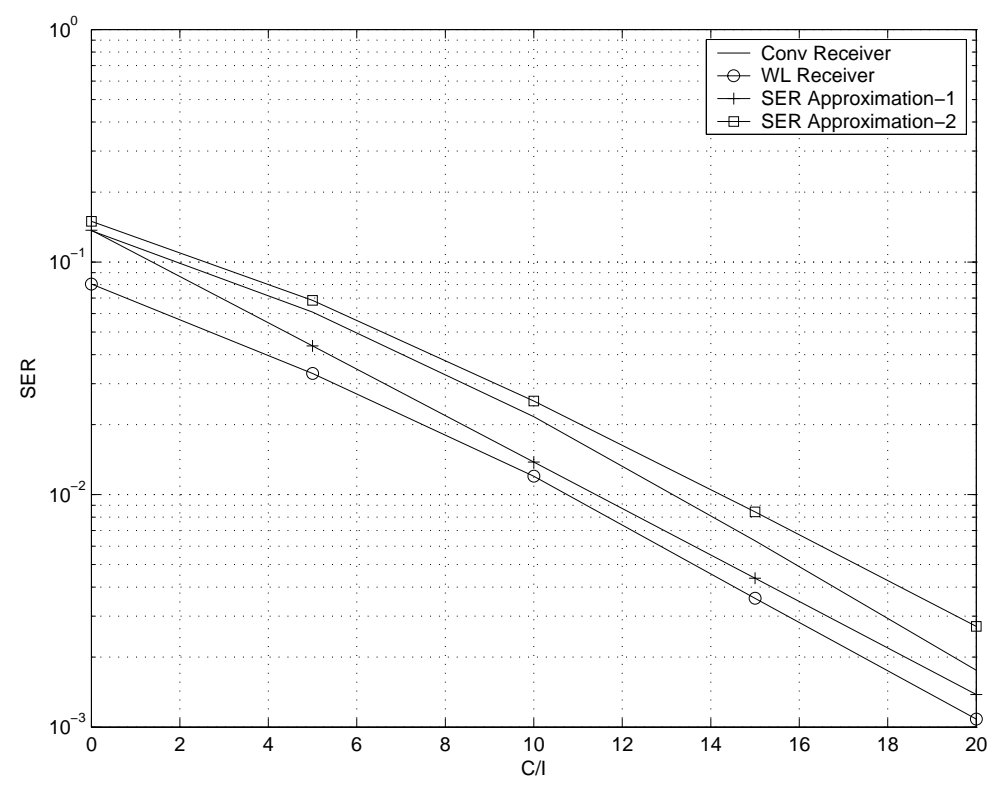

Figure 5.4. Single Antenna: BPSK desired-two BPSK interferers.

reaches an error floor (because it has no IC ability with a single antenna) whereas the WL receiver shows full IC gain with a diversity order equal to 0.5 . In this case, approximation-2 is a tight approximation for the whole range of SNR values whereas approximation-1 becomes a loose UB. In Figure 5.4 we plotted the SER for a single antenna receiver with 2-BPSK interferers as a function of $\mathrm{C} / \mathrm{I}$ where the $\mathrm{WL}$ receiver showed a limited IC gain (approximately $2.5 \mathrm{~dB}$ ) only. In this case, approximation-1 is a tight upper bound whereas approximation- 2 becomes some what loose UB. In Figure 5.5 we show the IC capability when a QPSK signal is corrupted by a single BPSK interferer. Note that, in this case, both approximations provide accurate results at high $\mathrm{C} / \mathrm{I}$ values whereas approximation-1 becomes a loose UB at low C/I. Since the IC capability is independent of desired modulation type, in the rest of the cases we show results for BPSK desired signal only.

When the number of antennas is two, the WL receiver can reject a maximum of 3-PAM interferers or a total of two mixed interferers (one PAM plus one QAM). In Figure 


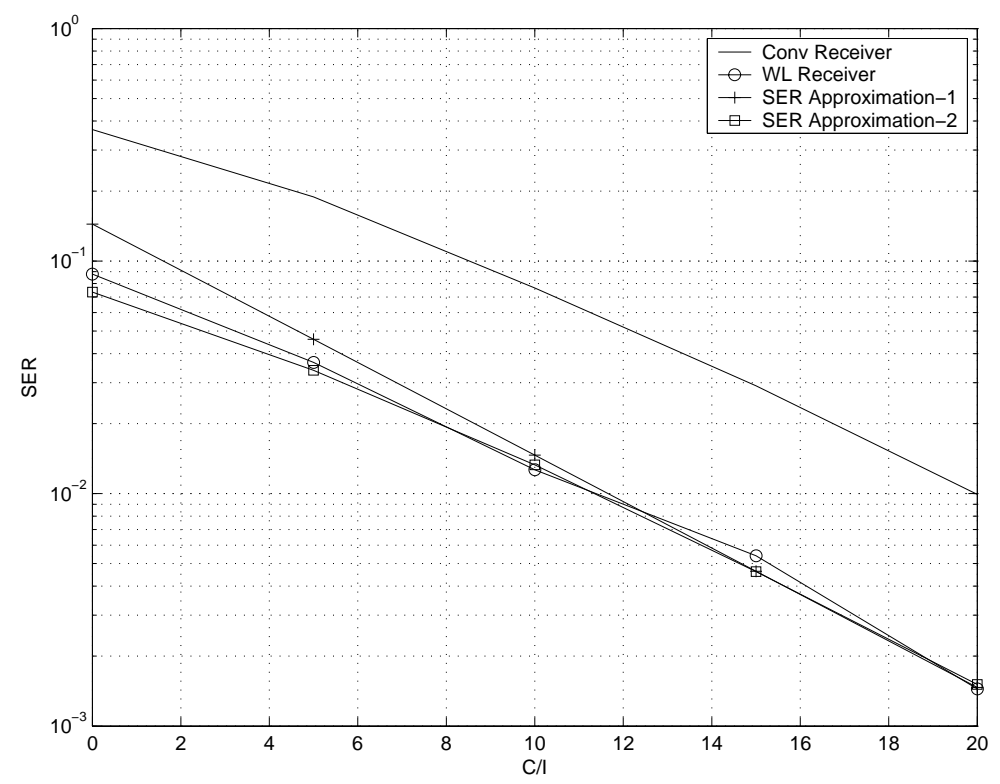

Figure 5.5. Single Antenna: QPSK desired-single BPSK CCI.

5.6 we show results for the mixed interferer case. The WL receiver shows complete IC effect as suggested by the analysis. In this case approximation-1 is becomes tight at high $\mathrm{C} / \mathrm{I}$ only whereas approximation- 2 under estimates the SER by about $2.5 \mathrm{~dB}$ for all C/I values. In Figure 5.7, we plotted the SER with 3-BPSK interferers as function of SNR. While the conventional receivers shows error floor, the WL receivers exhibits a performance gain with a diversity order equal to 0.5 . In this case approximation- 2 gives a better match to simulation than approximation-1. In general a combination of these approximations (a minimum of the two) gives a reasonable estimate of SER for most cases of interest.

\subsection{Summary}

In Rayleigh fading channels the real (I) and imaginary (Q) parts of the channel are assumed to be zero mean identical, and independent real Gaussian random variables. In this type of channel, WL receivers that exploit the I-Q parts of the signal (col- 


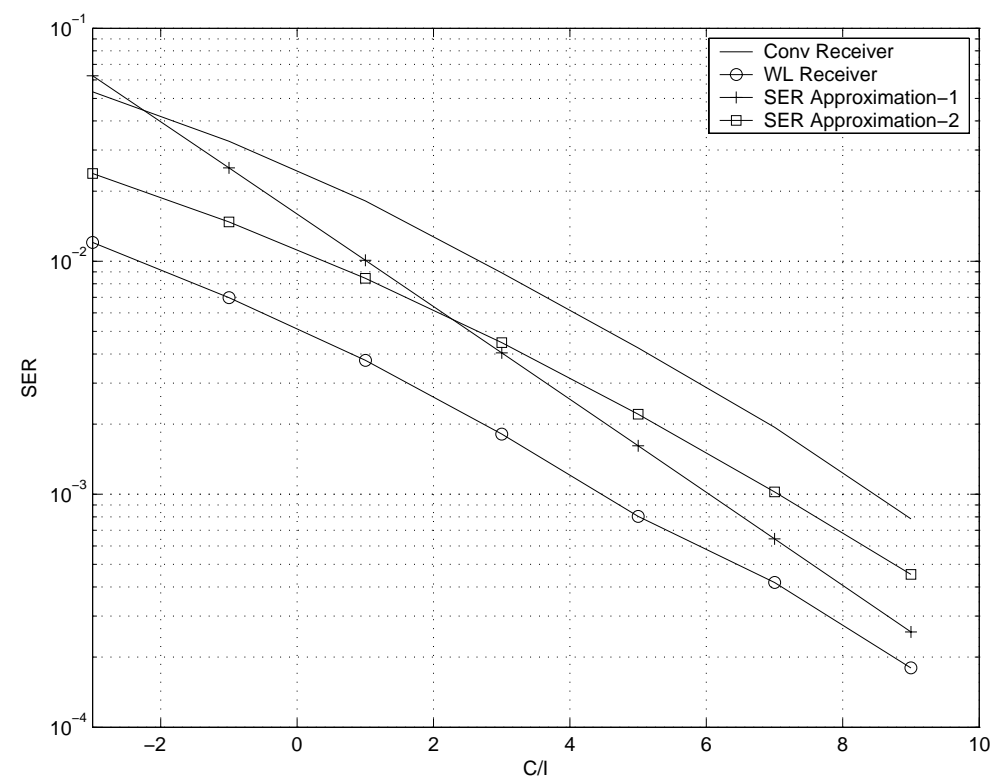

Figure 5.6. Two Antennas: BPSK desired-2 interferers (PAM+QAM).

lected at each antenna output) provide a significantly higher interference suppression gain than conventional MMSE receivers. When fading is symmetrically distributed between the I-Q parts (as in Rayleigh fading model), we show that the IC gain is independent of the modulation type (PAM/QAM) used by the desired signal but the gain depends mainly on the rank of the ICM denoted as $m$. In a system with $N$ antennas and I-Q split, the receiver has $2 N$ observations. The WL receiver is said to have full IC capability (that is complete interference removal) when the ICM is rank deficient i.e. when: $m<2 N$. The diversity order for this system is shown to be: $N-\frac{m}{2}$. This condition implies that, when the system employs a mixture of PAM and QAM signals, a WL receiver can reject any combination of $M_{1}$ PAM, $M_{2}$ QAM interferers satisfying the constraint: $M_{1}+2 M_{2}<2 N$, with a diversity of order: $N-\frac{M_{1}+2 M_{2}}{2}$. This result essentially says that WL receivers outperform conventional methods in both diversity and IC aspects. 


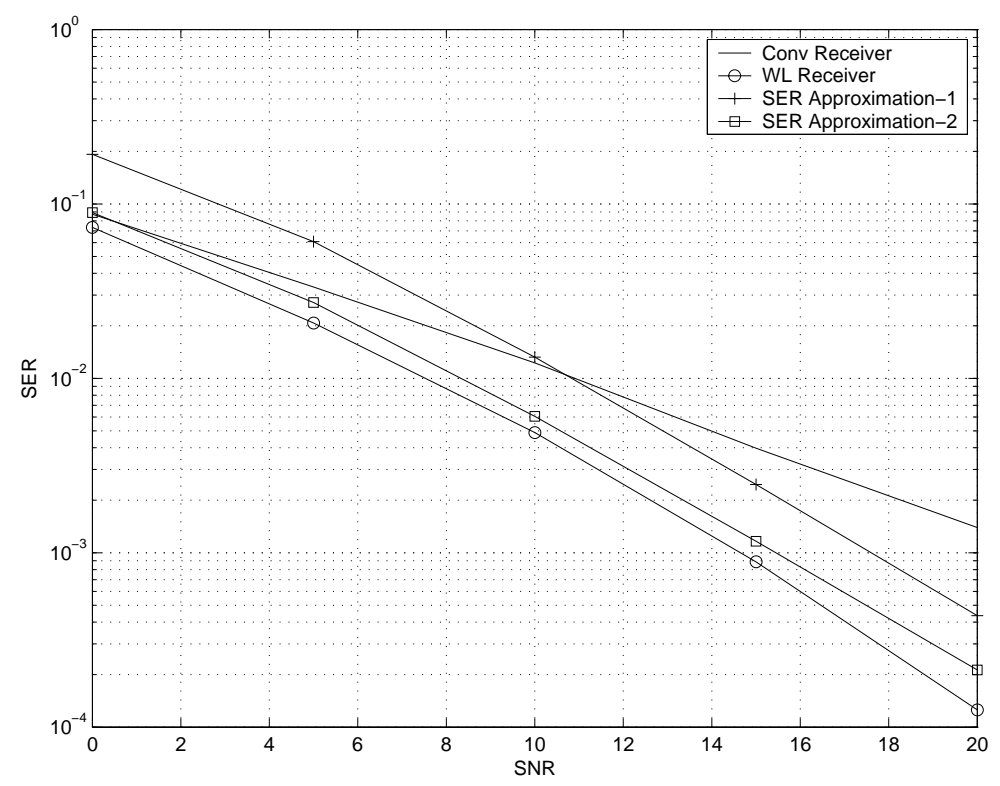

Figure 5.7. Two Antennas: BPSK desired-3 BPSK interferers $\left(C=I_{0}=1\right)$.

We obtained several approximations to the SER for M-ary PAM/QAM WL receiver with arbitrary number of antennas and arbitrary number of equal power interferers. The pseudo-determinant based approximation- 1 is a tight approximation when $N_{0}<<$ $I_{0}<<C$, for BPSK and QPSK signaling schemes. The trace based approximation-2 closely matched the simulation results for all values of interference and noise levels especially when the rank of ICM is less than $2 N$. We show that the combination of the two approximations provides a good approximation to the SER upper bound for most cases of interest. 


\section{CHAPTER 6}

\section{FUTURE WORK}

This thesis mainly considered applications of widely liner filtering concepts for enhancing the link performance in a TDMA-type wireless system. Our work can be extended in many directions.

- In Example 1 of Chapter 3, we showed that the WL-LE can tolerate significant ISI between I-Q branches without any performance loss when there is a 90 degree phase shift between the adjacent channel taps. In this context, let us consider a rotated PAM signal of type: $s(t)=\sum_{k=-\infty}^{k=\infty} j^{k} x_{k} p(t-k T)$ where $x_{k}$ is a real-valued modulation alphabet and $j^{k}$ is a deterministic phase rotation factor that introduces a 90-degrees phase shift between successive symbols and $p(t)$ is a real-valued pulse. Although $p(t)$ is real, because of $j^{k}$ rotation, the effective channel impulse response becomes complex after applying a $j^{-k}$ phase de-rotation to the received signal. For this type of scheme, the results of Chapter 3 indicates that an appropriate criterion for zero ISI channel design is:

$$
\tilde{\mathbf{h}}^{\dagger}(-f) \tilde{\mathbf{h}}(f)=\sum_{l=1}^{s}\left[\left|h_{I, l}(f)\right|^{2}+\left|h_{Q, l}(f)\right|^{2}=\text { Constant, } \quad-\frac{1}{2 T} \leq f \leq \frac{1}{2 T}(6.1\right.
$$

as opposed to the conventionally used metric:

$$
\mathbf{h}^{\dagger}(-f) \mathbf{h}(f)=\sum_{l=1}^{s}\left[\left|h_{l}(f)\right|^{2}=\text { Constant, } \quad-\frac{1}{2 T} \leq f \leq \frac{1}{2 T} .\right.
$$

In fact, as indicated by Example-1, modulation pulses with controlled amount of ISI (and better spectral properties) can be designed and reliably detected using a WL receivers with better noise resilience than conventional equalizers. 
Linearized GMSK modulation is one such example. We think that WLF is inherently related to the partial response ISI signal design problem for PAMtype systems.

- In Chapter 4 it was shown that, the QAM WL-DFE was not able to offer full IC benefit because of decision feedback errors. This loss may be reduced or even eliminated using more complex equalizers. Two possible solutions are listed below.

- For instance, reduced state sequence estimation (RSSE) methods can be used as an alternative to the WL-DFE symbol detector to reduce the loss caused by the feedback errors.

- In conventional systems, turbo equalization has been used successfully to combat the ISI introduced by pulse shaping filters/propagation channel. Similarly, a modification of the conventional MAP (or RSSE based) softin-soft-out [55] turbo receiver can be applied at the WL MMSE-DFE FFF output to reduce the error propagation problem. Also, as an alternative to trellis based turbo equalizers, a combination of the WL receivers concepts proposed in this thesis together with the MMSE turbo equalization methods proposed in [56] can be used to formulate practical, low-complex WL turbo receivers. Essentially, the turbo equalization problem can be formulated as an I-Q MMSE optimization with extrinsic information from channel decoder for complexity reduction.

- In Chapter 5 we considered the diversity-IC trade-off for flat fading channels only. The diversity order for a PAM/QAM WL receiver with $N$ antennas $M_{1}$ PAM, $M_{2}$ QAM interferers is shown to be:

$$
d_{W L}=N-M_{2}-\frac{M_{1}}{2}, \quad M_{1}+2 M_{2}<2 N .
$$


In frequency selective channels, we conjecture that, a PAM/QAM WL MMSEDFE receiver in the absence of error propagation, gives the following diversityIC trade-off:

$$
d_{W L}=\nu\left(N-M_{2}-\frac{M_{1}}{2}\right), \quad M_{1}+2 M_{2}<2 N
$$

where $\nu$ is the diversity order of the frequency selective channel under consideration. For PAM signaling, it is possible show that above result holds for an optimum MFB receiver. Therefore the DFE may be able to meet the given diversity-IC trade-off. However, for QAM signaling it is not straightforward to analyze the diversity performance. This is a subject for further research.

- Since WL receivers provide full IC gain flat fading channels, one can design an orthogonal multi-carrier scheme where each carrier meets the diversity-IC trade-off given by (6.3). As each sub-carrier in a OFDM system essentially undergoes flat fading, as fading across different frequencies becomes statistically independent (when there is enough separation), assuming there are $\nu$ sets of frequencies with independent fading, we can show that, a WL receiver meets the diversity-IC trade-off given by (6.4) when sufficient coding is applied across different carriers. Unlike, the DFE (that suffers from error propagation), this scheme ensures full IC but relies on a channel code to extract diversity from frequency selective channel. It may be useful compare WL MMSE-DFE receivers with WL multi-carrier receivers.

- In this thesis we assumed ideal knowledge of CSI and interference covariance information in analyzing the IC performance. These two quantities are usually estimated using a pilot sequence. In heavily dispersive channels, the quality of channel/interference covariance sets a limit to the achievable IC gain. In general, the length of the pilot sequence, the cross correlation between the pilots of signal 
and interference are the main impairments to the IC performance. Very few papers address the theoretical limits of IC with imperfect channel estimation. Moreover the basic trade-off between pilot overhead and IC gain has not been fully explored.

- Lastly, we would like to remark that WL equalizers are useful for low-complex implementation of multiple-input-multiple output (MIMO) systems as well. A potential MIMO application is the collaborative MIMO scheme such as the one proposed in the 3GPP UTRA release-7 (3.9G) uplink [57]. In this system, multiple users (with single antenna) communicate with the base station on the same frequency time-slot, while the receiver uses multiple antennas for signal separation. In this type of channel, WLE can provide advantage if one of the MIMO users has BPSK or PAM modulation. 


\section{CHAPTER 7}

\section{CONCLUSION}

This thesis illustrates the use of widely linear equalization concepts for enhancing link performance in TDMA-type wireless systems with ISI and CCI. When the wanted signal has QAM, splitting the complex baseband signal into real and imaginary parts leads to a multiple-input-multiple-output equalization problem (where we view the IQ parts of QAM symbol as independent signals) whereas in PAM case, I-Q split leads to a single-input-multiple-output (SIMO) equalization. For both cases, we introduce efficient WL MMSE/MMSE-DFE equalization structures for a system with multiple antennas and multiple co-channel interferers. Performance of proposed receivers is analyzed with various channel conditions.

First, we describe the benefits of WL equalization for PAM systems with ISI. When the ISI channel response is complex, we show that WL receivers have less noise enhancement and lower computational complexity compared to conventional equalizers. We derive closed form MSE expressions that demonstrate the advantage of new receivers for both finite and infinite length cases. Note that our results are applicable to other quadrature schemes such as offset-QPSK, MSK, and GMSK that have an equivalent PAM representation.

When the real and imaginary parts of the channel are distinct (as in Rayleigh fading channel), and when the signal and interference have distinct impulse responses, we show that WL-LE/DFE methods offer significantly better performance than conventional receivers. It is shown that WLE provides an IC gain especially when interference consists of PAM or a mixture of PAM and QAM signals. For both PAM and 
QAM signaling, assuming that the DFE feedback path is error free, we show that a WL receiver with $N$ antennas can reject up to $2 N-1$ PAM interferers or any combination of $M_{1}$ PAM and $M_{2}$ QAM interferers satisfying the constraint: $M_{1}+2 M_{2}<2 N$. Simulation results show that, in case of PAM signaling, both WL LE/DFE receivers offer full IC gain for any interference modulation type. However, with QAM signaling, in the presence of PAM-type interference, the gain of WL-DFE was limited by feedback errors while the IC benefit of WL-LE was affected by noise enhancement problem.

For the special case of flat Rayleigh fading, we have been able to compute several bounds (and approximations) to the SER of WL receivers with CCI. Two new results are shown. First, unlike frequency selective case, we show that IC ability of WL receivers is independent of modulation type (PAM/QAM) used by wanted signal but depends mainly on the modulation type employed by individual interferers and the total number of antennas. Secondly, assuming that interference consists of a mixture of PAM and QAM signals, we show that, a PAM/QAM WL receiver can reject any combination of $M_{1}$ PAM, $M_{2}$ QAM interferers satisfying the constraint: $M_{1}+2 M_{2}<2 N$ while preserving a diversity of order: $N-\frac{M_{1}}{2}-M_{2}$. Note that, a conventional MMSE receiver with $N$ antennas and $M$ interferers $(M<N)$ can suppress a maximum of $M$ interferers with a diversity order $N-M$. The above results basically implies that WL receivers provide a diversity advantage in addition to IC gain.

Also, the complexity analysis for interference limited channels indicates that the IC gain comes at the expense of an increase in receiver complexity. We think that the present systems can very well handle the additional computation required for WL processing. Therefore, the proposed receivers are useful for application in wireless systems that use PAM or a combination of PAM and QAM schemes. 
Finally, we would like to remark here that the conventional WL receiver implementation based on complex, complex-conjugate parts of the received signal requires more complexity as it requires complex arithmetic in both filter tap calculation and signal filtering whereas the proposed I-Q approach is computationally more efficient as it involves real arithmetic only. The difference between the two approaches becomes even more pronounced in a finite length implementation. 
APPENDIX A

MSE EXPRESSIONS FOR DFE 


\section{A.1 MSE Relationship for Infinite Length DFE}

Using (3.41), (3.13) we can express the MSE for conventional DFE as

$$
\begin{aligned}
\text { MSE }_{\text {Conv DFE }}=e^{-T \int_{-\frac{1}{2 T}}^{\frac{1}{2 T}} \ln \left[1+\frac{2|\mathbf{h}(f)|^{2}}{N_{0}}\right] d f} & =\frac{N_{0}}{2} e^{T \int_{-\frac{1}{2 T}}^{\frac{1}{2 T}} \ln \frac{1}{a(f)-\bar{a}(f)} d f} \\
& =\frac{N_{0}}{2} e^{T \int_{-\frac{1}{2 T}}^{\frac{1}{2 T}} \ln \frac{1}{a(f)} d f-T \int_{-\frac{1}{2 T}}^{\frac{1}{2 T}} \ln \left[1-\frac{\bar{a}(f)}{a(f)}\right] d f} \\
& =\frac{N_{0}}{2} e^{T \int_{-\frac{1}{2 T}}^{\frac{1}{2 T}} \ln \frac{1}{a(f)} d f+T \int_{-\frac{1}{2 T}}^{\frac{1}{2 T}}\left[\frac{\bar{a}(f)}{a(f)}+\frac{\bar{a}^{2}(f)}{2 a^{2}(f)}+. .\right] d f} \\
& =\frac{N_{0}}{2} e^{T \int_{-\frac{1}{2 T}}^{\frac{1}{2 T}} \ln \frac{1}{a(f)} d f+T \int_{-\frac{1}{2 T}}^{\frac{1}{2 T}}\left[\frac{\bar{a}^{2}(f)}{2 a^{2}(f)}+\frac{\bar{a}^{4}(f)}{4 a^{4}(f)}+. .\right] d f} .
\end{aligned}
$$

On line 3 we invoked an infinite series expansion $-\ln (1-x)=\sum_{k=1}^{\infty} \frac{x^{k}}{k}$ for $|x|<1$.

In line 4 , we excluded the odd powers of $\bar{a}(f)$ as they become zero when integrated. Using (3.47), the MSE for WL-DFE can be expressed as:

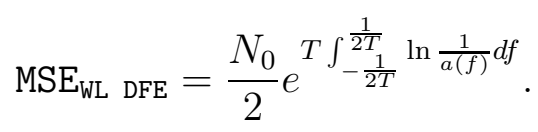

Using this we can expresses the MSE metrics as:

$$
\mathrm{MSE}_{\text {Conv DFE }}=\operatorname{MSE}_{\mathrm{WL} \mathrm{DFE}} e^{T \int_{-\frac{1}{2 T}}^{\frac{1}{2 T}}\left[\frac{\bar{a}^{2}(f)}{2 a^{2}(f)}+\frac{\bar{a}^{4}(f)}{4 a^{4}(f)}+. .\right] d f} .
$$

Since even powers of $\frac{\bar{a}(f)}{a(f)}$ are positive, we get:

$$
\mathrm{MSE}_{\mathrm{Conv} \text { DFE }} \geq \mathrm{MSE}_{\mathrm{WL} \text { DFE }}
$$

\section{A.2 Proof of Theorem 3.3.1}

The first two statements can be verified by writing $\mathbf{Q}$ in terms of real and imaginary parts of $\mathbf{H}$. To prove the third statement, consider $|\mathbf{Q}|=|\mathbf{A}|\left|\mathbf{I}+j \mathbf{A}^{-1} \mathbf{B}\right|$. Since both $\mathbf{Q}$ and $\mathbf{A}$ are positive definite $\mathbf{I}+j \mathbf{A}^{-1} \mathbf{B}$ is also positive definite and hence all its eigenvalues are real and greater than -1 . Let $\lambda$ be an eigenvalue of $j \mathbf{A}^{-1} \mathbf{B}$ and $x$ be the corresponding eigenvector. Then we have $j \mathbf{A}^{-1} \mathbf{B} x=\lambda x$. Conjugating both 
sides, we get $j \mathbf{A}^{-1} \mathbf{B} x^{*}=-\lambda x^{*}$. We see that $-\lambda$ is also an eigenvalue and $x^{*}$ is the corresponding eigenvector. Since all the eigenvalues are greater than -1 and they must appear in pairs $(-\lambda, \lambda)$ and be confined to the range $(-1,1)$. We can also see that $\left|j \mathbf{A}^{-1} \mathbf{B}\right|$ is restricted to the interval $[0,1)$. Further we can write $\left|\mathbf{I}+j \mathbf{A}^{-1} \mathbf{B}\right|$ as $\prod^{k}\left(1-\lambda_{k}^{2}\right)$. Since each $\lambda_{k}<1$ we see that $0<\left|\mathbf{I}+j \mathbf{A}^{-1} \mathbf{B}\right| \leq 1$ which proves the fourth statement. Now let us consider the eigen-structure of $\mathbf{C}^{m} \mathbf{A}^{-1}$. Since the eigenvalues of $\mathbf{C}^{m}$ can be obtained by raising the eigenvalues of $\mathbf{C}^{m}$ to the $m$ th power, $\left[(-\lambda)^{m}, \lambda^{m}\right]$ is an eigen pair for $\mathbf{C}^{m}$. We can see that these eigenvalues become positive for even values of $m$ and remain a negated pair for $m$ odd. Since $\mathbf{A}^{-1}$ is positive definite and $\mathbf{C}^{m}$ is positive semi-definite for $m$ even, $\mathbf{C}^{m} \mathbf{A}^{-1}$ is also positive semi definite and hence its eigen values are greater than or equal to zero. For odd values of $m, \mathbf{C}^{m} \mathbf{A}^{-1}$ becomes a skew-Hermitian matrix with purely imaginary entries [52]; consequently its diagonal entries must be zero.

\section{A.3 Exact MSE Relationship between Finite Length Conventional and WL DFE Receivers}

Recall that the conventional MMSE-DFE solution involves Choleski factorization of form (3.49)

$$
\mathbf{R}_{x x}^{-1}+\mathbf{H}^{\dagger} \mathbf{R}_{n n}^{-1} \mathbf{H}=\mathbf{L D L}^{\dagger}=\mathbf{A}+j \mathbf{B}
$$

Let $\mathbf{L}=\mathbf{L}_{a}+j \mathbf{L}_{b}$. We can express A.1 in terms of $\mathbf{L}_{a}, \mathbf{L}_{b}$ as:

$$
\mathbf{A}+j \mathbf{B}=\left[\mathbf{L}_{a} \mathbf{D L}_{a}^{\dagger}+\mathbf{L}_{b} \mathbf{D L}_{b}^{\dagger}\right]+j\left[\mathbf{L}_{b} \mathbf{D} \mathbf{L}_{a}^{\dagger}-\mathbf{L}_{a} \mathbf{D L}_{b}^{\dagger}\right]
$$

For the WL case we have:

$$
\mathbf{R}_{x x}^{-1}+\tilde{\mathbf{H}}^{\dagger} \mathbf{R}_{\tilde{n} \tilde{n}}^{-1} \tilde{\mathbf{H}}=\tilde{\mathbf{L}} \tilde{\mathbf{D}} \tilde{\mathbf{L}}^{\dagger}=\mathbf{A}
$$


Combining (A.1), (A.2) and expressing $\mathbf{A}=\mathbf{L}_{a} \mathbf{D L}_{a}^{\dagger}+\mathbf{L}_{b} \mathbf{D} \mathbf{L}_{b}^{\dagger}$ in terms of its real and imaginary parts as we get

$$
\begin{aligned}
\tilde{\mathbf{L}} \tilde{\mathbf{D}} \tilde{\mathbf{L}}^{\dagger} & =\mathbf{L}_{a} \mathbf{D} \mathbf{L}_{a}^{\dagger}+\mathbf{L}_{b} \mathbf{D} \mathbf{L}_{b}^{\dagger} \\
\Longrightarrow \tilde{\mathbf{D}} & =\tilde{\mathbf{L}}^{-1} \mathbf{L}_{a} \mathbf{D} \mathbf{L}_{a}^{\dagger} \tilde{\mathbf{L}}^{\dagger-1}+\tilde{\mathbf{L}}^{-1} \mathbf{L}_{b} \mathbf{D} \mathbf{L}_{b}^{\dagger} \tilde{\mathbf{L}}^{\dagger-1}=\mathbf{G}_{a} \mathbf{D} \mathbf{G}_{a}^{\dagger}+\mathbf{G}_{b} \mathbf{D} \mathbf{G}_{b}^{\dagger}
\end{aligned}
$$

where $\mathbf{G}_{a}=\tilde{\mathbf{L}}^{-1} \mathbf{L}_{a}, \mathbf{G}_{b}=\tilde{\mathbf{L}}^{-1} \mathbf{L}_{b}$. Note that $\mathbf{L}_{a}$ is a lower triangular (LT) matrix with unit diagonal and $\mathbf{L}_{b}$ is also LT with all zeros along the main diagonal. Since $\tilde{\mathbf{L}}^{-1}$ is LT unit diagonal, $\mathbf{G}_{a}$ becomes LT with unit diagonal while $\mathbf{G}_{b}$ becomes LT with zeros on the main diagonal. Since $\mathbf{A}+j \mathbf{B}$ is positive definite $(\mathrm{PD})$, both $\mathbf{D}, \tilde{\mathbf{D}}$ are both $\mathrm{PD}$. We see that $\mathbf{G}_{a} \mathbf{D} \mathbf{G}_{a}^{\dagger}+\mathbf{G}_{b} \mathbf{D} \mathbf{G}_{b}^{\dagger}$ sum up to a PD diagonal matrix $\tilde{\mathbf{D}}$. Let us denote the diagonal entries $\mathbf{D}_{a}=\operatorname{diag}\left[\mathbf{G}_{a} \mathbf{D} \mathbf{G}_{a}^{\dagger}\right]$ and $\mathbf{D}_{b}=\operatorname{diag}\left[\mathbf{G}_{b} \mathbf{D} \mathbf{G}_{b}^{\dagger}\right]$. Since $\mathbf{G}_{a}$ is $\mathrm{LT}$ with unit diagonal, each of the diagonal entries of $\mathbf{D}_{a}$ must be greater than or equal the corresponding entries in $\mathbf{D}$. On the other hand, the elements of $\mathbf{D}_{b}$ take positive values (lower than the corresponding entries in $\mathbf{D}_{a}$ ). Now we have the desired result. Each of the diagonal entries of $\tilde{\mathbf{D}}$ is greater than or equal to that of the corresponding entries in $\mathbf{D}$, that is

$$
\tilde{d}_{m} \geq d_{m}, \quad m \geq 0
$$

Now we have the desired result. Each of the diagonal entries of $\tilde{\mathbf{D}}$ is greater than or equal to that of the corresponding entries in $\mathbf{D}$. 
APPENDIX B

SPECTRUM FACTORIZATION 


\section{B.1 Spectrum Factorization Algorithm}

Recall that in order to determine MMSE-DFE settings we had to calculate the feedback filter given by a spectral factorization of form:

$$
\mathbf{S}(f)=\mathbf{L}^{\dagger}(-f) \mathbf{D L}(f)
$$

where $\mathbf{L}(f)$ is causal, monic, and minimum phase filter with all its poles and zeros inside the unit circle. Note that we need to perform matrix spectrum factorization in case of QAM WL MMSE-DFE and scalar factorization in other cases. The presented algorithms applies to both cases. Note that the algorithm can also be used to design a whitening filter to whiten the noise plus interference correlation matrix in case of colored noise.

\section{B.1.1 Spectral Factorization using State-Space Models}

The derivations given in this section are obtained from the survey paper by A. Sayed and T. Kailath [58]. For detailed derivations the reader is referred to Chapter-11 of Linear Estimation by T. Kailath et.al. Using D-domain notation:

$$
\mathbf{S}(D)=\mathbf{L}(D) \mathbf{D L}^{\dagger}\left(D^{-*}\right)
$$

where $\mathbf{L}(D)$ is a matrix valued polynomial of order $m$ with all its roots inside the unit circle. Let,

$$
\mathbf{L}(D)=\mathbf{I}+\sum_{k=1}^{m} \mathbf{L}_{k} D^{k}
$$

Then

$$
\mathbf{L}^{\dagger}\left(D^{-*}\right)=\mathbf{I}+\sum_{k=1}^{m} \mathbf{L}_{k}^{\dagger} D^{-k}
$$


Let $\mathbf{Z}_{k}$ be a random process which produces the desired D-domain spectrum $\mathbf{S}(D)$ with a covariance sequence given by

$$
\begin{aligned}
E\left[\mathbf{Z}_{k} \mathbf{Z}_{k-j}^{\dagger}\right] & =\mathbf{P}_{j}, \quad 0 \leq j \leq m \\
& =\mathbf{P}_{-j}^{\dagger}, \quad 0<j \leq-m \\
& =0, \quad \text { Otherwise. }
\end{aligned}
$$

Now we can view $\mathbf{Z}_{k}$ as the output of a linear time invariant filter $\mathbf{L}^{\dagger}\left(D^{-*}\right)$ driven by a white noise sequence say $\mathbf{E}_{k}$. The sequence $\mathbf{E}_{k}$ is also known as the innovations process associated with $\mathbf{Z}_{k}$ since it is related to $\mathbf{Z}_{k}$ through a causal and causally invertible transformation $\mathbf{L}^{\dagger}\left(D^{-*}\right)$. We can express $\mathbf{Z}_{k}$ as

$$
\mathbf{Z}_{k}=\mathbf{E}_{k}+\mathbf{L}_{1}^{\dagger} \mathbf{E}_{k-1}+\mathbf{L}_{2}^{\dagger} \mathbf{E}_{k-2}+. .+\mathbf{L}_{m}^{\dagger} \mathbf{E}_{k-m}
$$

Now we can cast the problem in state-space form as

$$
\begin{aligned}
\mathbf{X}_{k+1} & =\mathbf{F X}_{k}+\overline{\mathbf{K}} \mathbf{E}_{k} \\
\mathbf{Z}_{k} & =\mathbf{H X}_{k}+\mathbf{E}_{k}
\end{aligned}
$$

where

$$
\mathbf{F} \triangleq\left[\begin{array}{ccccc}
0 & & & & \\
\mathbf{I} & 0 & & & \\
& & \ddots & \ddots & \\
& & & \mathbf{I} & 0
\end{array}\right], \quad \mathbf{H} \triangleq[0, . ., 0, \mathbf{I}], \quad \overline{\mathbf{K}} \triangleq\left[\mathbf{L}_{1}^{\dagger}, \mathbf{L}_{2}^{\dagger}, . ., \mathbf{L}_{m}^{\dagger}\right]^{\prime}
$$

Also define

$$
\overline{\mathbf{N}} \triangleq\left[\mathbf{P}_{m}, \mathbf{P}_{m-1}, . ., \mathbf{P}_{1}\right]^{\prime}
$$

The transfer function from $\mathbf{E}_{k}$ to $\mathbf{Z}_{k}$ is given by

$$
\mathbf{T}(D)=\mathbf{I}+\mathbf{H}[D \mathbf{I}-\mathbf{F}]^{-1} \overline{\mathbf{K}}=\mathbf{L}^{\dagger}\left(D^{-*}\right)
$$


Using matrix inversion lemma we can also show that

$$
\mathbf{L}^{\dagger-\mathbf{1}}\left(D^{-*}\right)=\mathbf{I}-\mathbf{H}[D \mathbf{I}-(\mathbf{F}-\overline{\mathbf{K}} \mathbf{H})]^{-1} \overline{\mathbf{K}}
$$

so that the matrix $\mathbf{F}-\overline{\mathbf{K}} \mathbf{H}$ must have all its eigenvalues inside the unit circle.

\section{B.1.1.1 CKMS Algorithm}

The spectral factors can be directly computed by using so-called ChandrasekharKailath-Morf-Sidhu (CKMS) recursions very efficiently. The algorithm is summarized in the following steps.

1. Define the initial conditions: $\mathbf{L}_{0}=\overline{\mathbf{N}}=\overline{\mathbf{K}}$ and $\mathbf{R}_{e, 0}=\mathbf{P}_{0}=\mathbf{R}_{r, 0}$.

2. Iterate the following recursions for $i \geq 0$

- $\mathbf{K}_{i+1}=\mathbf{K}_{i}-\mathbf{F L}_{i} \mathbf{R}_{r, i}^{-1} \mathbf{L}_{i}^{\dagger} \mathbf{H}^{\dagger}$

- $\mathbf{L}_{i+1}=\mathbf{F L}_{i}-\mathbf{K}_{i} \mathbf{R}_{e, i}^{-1} \mathbf{H L}_{i}$

- $\mathbf{R}_{e, i+1}=\mathbf{R}_{e, i}-\mathbf{H L}_{i} \mathbf{R}_{r, i}^{-1} \mathbf{L}_{i}^{\dagger} \mathbf{H}^{\dagger}$

- $\mathbf{R}_{r, i+1}=\mathbf{R}_{r, i}-\mathbf{L}_{i}^{\dagger} \mathbf{H}^{\dagger} \mathbf{R}_{e, i}^{-1} \mathbf{H L}_{i}$

3. Then in the limit as $i \rightarrow \infty, \mathbf{K}_{i}$ tends to a constant and $\mathbf{R}_{e, i}$ tends to a constant $\mathbf{R}_{e}$. Further, let $\overline{\mathbf{K}}=\mathbf{K R}_{e}^{-1}$.

4. The desired $\mathbf{D}, \mathbf{L}^{\dagger}\left(D^{-*}\right)$ are given by $\mathbf{D}=\mathbf{R}_{e}$, and

$$
\mathbf{L}^{\dagger}\left(D^{-*}\right)=\mathbf{I}+[D \mathbf{I}-\mathbf{F}]^{-1} \overline{\mathbf{K}}
$$

More explicitly, the individual entries of $\overline{\mathbf{K}}$ are the coefficients of $\mathbf{L}^{\dagger}\left(D^{-*}\right)$, i.e.,

$$
\overline{\mathbf{K}}=\left[\mathbf{L}_{1}^{\dagger}, \mathbf{L}_{2}^{\dagger}, . ., \mathbf{L}_{m}^{\dagger}\right]^{\prime}
$$

5. Next $\mathbf{L}^{\dagger-1}\left(D^{-*}\right)$ can be calculated as

$$
\mathbf{L}^{\dagger-\mathbf{1}}\left(D^{-*}\right)=\mathbf{I}-\mathbf{H}[D \mathbf{I}-(\mathbf{F}-\overline{\mathbf{K}} \mathbf{H})]^{-1} \overline{\mathbf{K}}
$$


More explicitly, each tap in $\mathbf{L}^{\dagger-\mathbf{1}}\left(D^{-*}\right)$ denoted as $\mathbf{L}^{\dagger-\mathbf{1}}(k)$, is given by

$$
\mathbf{L}^{\dagger-\mathbf{1}}(k)=-\mathbf{H} \overline{\mathbf{F}}^{(k-1)} \overline{\mathbf{K}}
$$

where $\overline{\mathbf{F}} \triangleq \mathbf{F}-\overline{\mathbf{K}} \mathbf{H}$, and $k=1,2, . ., L$. Note that the $\mathbf{L}^{\dagger-\mathbf{1}}\left(D^{-*}\right)$ is an infinite length polynomial but in practice it will be truncated to a finite length $L$ for implementation purpose. 
APPENDIX C

IC PROPERTIES 


\section{C.1 IC Properties of Conventional Receivers}

Consider the limiting behavior of $\mathbf{h}^{\dagger}(-f) \mathbf{R}_{\mathbf{n n}}^{-\mathbf{1}}(f) \mathbf{h}(f)$ as $N_{0} \rightarrow 0$. From (2.23), recall that $\mathbf{R}_{\mathbf{n n}}(f)=\mathbf{R}_{\mathbf{i i}}(f)+s N_{0} \mathbf{I}$. Assuming an over-sampling factor of $s=1$, let $r_{c}$ denotes the rank of $N \times N$ interference correlation matrix $\mathbf{R}_{\mathbf{i i}}(f)$. Then its first $N-r_{c}$ eigenvalues are zero and the remaining $r_{c}$ eigenvalues, denoted as $\lambda_{k}(f), k=$ $N-r_{c}+1, . ., N$, are non-zero. Hence, the eigenvalues of noise plus interference correlation matrix: $\mathbf{R}_{\mathbf{n n}}(f)=\sum_{m=1}^{M} \mathbf{g}^{m}(f) \mathbf{g}^{m \dagger}(-f)+N_{0} \mathbf{I}$ are given by:

$$
\begin{aligned}
\bar{\lambda}_{k}(f) & =N_{0}, \quad k=1, . ., N-r_{c} \\
& =\lambda_{k}(f)+N_{0}, \quad k=N-r_{c}+1, . ., N .
\end{aligned}
$$

Let us represent the matrix $\mathbf{R}_{\mathbf{n n}}^{-\mathbf{1}}(f)$ as:

$$
\mathbf{R}_{\mathbf{n n}}^{-\mathbf{1}}(f)=\mathbf{U}^{\dagger}(-f) \Delta(f) \mathbf{U}(f)
$$

where $\mathbf{U}(f)$ is a unitary matrix and $\Delta(f)$ is a diagonal matrix given by:

$$
\Delta(f)=\operatorname{diag}\left[\frac{1}{\bar{\lambda}_{1}(f)}, . ., \frac{1}{\bar{\lambda}_{N}(f)}\right] .
$$

Using (C.1), we can express the frequency dependent SNR term as

$$
\begin{aligned}
\mathbf{h}^{\dagger}(-f) \mathbf{R}_{\mathbf{n n}}^{-\mathbf{1}}(f) \mathbf{h}(f) & =\omega^{\dagger}(-f) \Delta(f) \omega(f), \text { where } \omega(f)=\mathbf{U}(f) \mathbf{h}(f) \\
& =\frac{1}{N_{0}} \sum_{k=1}^{N-r_{c}} \omega_{k}^{2}(f)+\sum_{k=N-r_{c}+1}^{N-1} \frac{\omega_{k}^{2}(f)}{\bar{\lambda}_{k}(f)}
\end{aligned}
$$

Next we determine the rank structure of $\mathbf{R}_{\mathbf{n n}}(f)$. Recall that the interference correlation matrix (ICM) can be represented in frequency domain as (2.23):

$$
\mathbf{R}_{\mathbf{i i}}(f)=\sum_{m=1}^{M} \mathbf{g}^{m}(f) \mathbf{g}^{m \dagger}(-f) .
$$

Since $\mathbf{g}^{m}(f)$ is $N \times 1$ vector, if the number of interferers $M$ is less $N$, the rank becomes $r_{c}=M$. If $M \geq N$, the correlation matrix has full rank i.e. $r_{c}=M$. Combining these 
two results, we get: $r_{c}=\min (N, M)$. Let us assume that the number of interferers $M$ is less $N$. In that case, the denominator of the first term in (C.2) will be inversely proportional to $N_{0}$. If we assume that the numerator term $\sum_{k=1}^{N-r_{c}} \omega_{k}^{2}(f)$ is a non-zero quantity, as $N_{0} \rightarrow 0$, we can see that $\mathbf{h}^{\dagger}(-f) \mathbf{R}_{\mathbf{n n}}^{-\mathbf{1}}(f) \mathbf{h}(f) \rightarrow \infty$. Therefore, we can say that the receiver provides an infinite SNR gain when the ICM is rank deficient. Alternatively we can say that: a conventional receiver with $N$ antennas completely eliminates $M$ interferers $(M<N)$ as long as $\sum_{k=1}^{N-r_{c}} \omega_{k}^{2}(f)$ takes finite non-zero values. In random channels, when the channel state information of signal and interference are statistically independent continuous random variables, the singular condition: $\sum_{k=1}^{N-r_{c}} \omega_{k}^{2}(f)=0$ occurs with very small probability. Thus we get an infinite SNR gain with probability nearly equal to 1 . However, when the ICM has full rank i.e. when $M \geq N$, the receiver can provide a partial IC gain only.

\section{C.2 IC Properties of QAM WL MMSE-DFE Receiver}

Let us express the channel matrix $\tilde{\mathbf{H}}^{\dagger}(-f)$ as $\tilde{\mathbf{H}}^{\dagger}(-f)=\left[\begin{array}{c}\tilde{\mathbf{h}}^{\dagger}(-f) \\ \tilde{\mathbf{h}}^{\dagger}(-f) \mathbf{P}\end{array}\right]$ where $\mathbf{P} \cong$

$$
\begin{aligned}
& {\left[\begin{array}{cc}
0 & \mathbf{I} \\
-\mathbf{I} & 0
\end{array}\right] \text {. We expresses the SNR in terms of } \mathbf{P} \text { as: }} \\
& \tilde{\mathbf{H}}^{\dagger}(-f) \mathbf{R}_{\tilde{\mathbf{n}} \tilde{\mathbf{n}}}^{-\mathbf{1}}(f) \tilde{\mathbf{H}}(f)=\left[\begin{array}{cc}
\tilde{\mathbf{h}}^{\dagger}(-f) \mathbf{R}_{\tilde{\mathbf{n}} \tilde{\mathbf{n}}}^{-1}(f) \tilde{\mathbf{h}}(f) & \tilde{\mathbf{h}}^{\dagger}(-f) \mathbf{R}_{\tilde{\mathbf{n}} \tilde{\mathbf{n}}}^{-1}(f) \mathbf{P}^{\dagger} \tilde{\mathbf{h}}(f) \\
\tilde{\mathbf{h}}^{\dagger}(-f) \mathbf{P} \mathbf{R}_{\tilde{\mathbf{n}} \tilde{\mathbf{n}}}^{-\mathbf{1}}(f) \tilde{\mathbf{h}}(f) & \tilde{\mathbf{h}}^{\dagger}(-f) \mathbf{P} \mathbf{R}_{\tilde{\mathbf{n}} \tilde{\mathbf{n}}}^{-\mathbf{1}}(f) \mathbf{P}^{\dagger} \tilde{\mathbf{h}}(f)
\end{array}\right]
\end{aligned}
$$

Let us assume that over-sampling factor $s=1$. The determinant of (C.3) can be compactly expressed as:

$$
\begin{gathered}
\left|\tilde{\mathbf{H}}^{\dagger}(-f) \mathbf{R}_{\tilde{\mathbf{n}} \tilde{\mathbf{n}}}^{-\mathbf{1}}(f) \tilde{\mathbf{H}}(f)\right|=\left(\tilde{\mathbf{h}}^{\dagger}(-f) \mathbf{R}_{\tilde{\mathbf{n}} \tilde{\mathbf{n}}}^{-\mathbf{1}}(f) \tilde{\mathbf{h}}(f)\right)\left(\tilde{\mathbf{h}}^{\dagger}(-f) \mathbf{P} \mathbf{R}_{\tilde{\mathbf{n}} \tilde{\mathbf{n}}}^{-\mathbf{1}}(f) \mathbf{P}^{\dagger} \tilde{\mathbf{h}}(f)\right)- \\
\left|\tilde{\mathbf{h}}^{\dagger}(-f) \mathbf{R}_{\tilde{\mathbf{n}} \tilde{\mathbf{n}}}^{-\mathbf{1}}(f) \mathbf{P}^{\dagger} \tilde{\mathbf{h}}(f)\right|^{2} .
\end{gathered}
$$


To evaluate the asymptotic behavior of (C.4), we express this quantity in terms of eigenvalues of $\mathbf{R}_{\tilde{\mathbf{n}} \tilde{\mathbf{n}}}^{-\mathbf{1}}(f)$. From (2.25), the $2 N \times 2 N$ noise plus interference correlation matrix was expressed as: $\mathbf{R}_{\tilde{\mathbf{n}} \tilde{\mathbf{n}}}(f)=\mathbf{R}_{\tilde{\mathbf{i}}}(f)+\frac{N_{0}}{2} \mathbf{I}$. Let $r$ denote the rank of $\mathbf{R}_{\widetilde{\mathbf{i}}}(f)$. Then $\mathbf{R}_{\widetilde{\mathbf{i}}}(f)$ has $r$ non-zero (positive) eigenvalues and the remaining $2 N-r$ eigenvalues are exactly zero. Next, we consider unitary decomposition:

$$
\mathbf{R}_{\tilde{\mathbf{n}} \tilde{\mathbf{n}}}^{-\mathbf{1}}(f)=\tilde{\mathbf{U}}^{\dagger}(-f) \tilde{\Delta}(f) \tilde{\mathbf{U}}(f)
$$

where $\tilde{\mathbf{U}}(f)$ is a unitary matrix and $\tilde{\Delta}(f)$ is a diagonal matrix with entries:

$$
\begin{aligned}
\tilde{\lambda}_{k}(f) & =\frac{2}{N_{0}}, \text { for } k=1, . ., 2 N-r \\
& =\frac{1}{\hat{\lambda}_{k}(f)+\frac{N_{0}}{2}}, \text { for } k=2 N-r+1, . ., 2 N
\end{aligned}
$$

where $\hat{\lambda}_{k}(f)$ are the non-zero eigenvalues of $\mathbf{R}_{\widetilde{\mathbf{i}}}(f)$. Using (C.5), we express the first diagonal element of (C.3) as:

$$
\begin{aligned}
\tilde{\mathbf{h}}^{\dagger}(-f) \mathbf{R}_{\tilde{\mathbf{n}} \tilde{\mathbf{n}}}^{-\mathbf{1}}(f) \tilde{\mathbf{h}}(f) & =\tilde{\omega}^{\dagger}(-f) \tilde{\Delta}(f) \tilde{\omega}(f), \quad \text { where } \tilde{\omega}(f)=\tilde{\mathbf{U}}(f) \tilde{\mathbf{h}}(f) \\
& =\frac{2}{N_{0}} \sum_{k=1}^{2 N-r} \tilde{\omega}_{k}^{2}(f)+\sum_{k=2 N-r+1}^{2 N} \frac{\tilde{\omega}_{k}^{2}(f)}{\tilde{\lambda}_{k}(f)}
\end{aligned}
$$

Using (C.5), the second diagonal element of (C.3) can be expressed as:

$$
\begin{aligned}
\tilde{\mathbf{h}}^{\dagger}(-f) \mathbf{P} \mathbf{R}_{\tilde{\mathbf{n}} \tilde{\mathbf{n}}}^{-\mathbf{1}}(f) \mathbf{P}^{\dagger} \tilde{\mathbf{h}}(f) & =\bar{\omega}^{\dagger}(-f) \tilde{\Delta}(f) \bar{\omega}(f), \text { where } \bar{\omega}(f)=\tilde{\mathbf{U}}(f) \mathbf{P}^{\dagger} \tilde{\mathbf{h}}(f) \\
& =\frac{2}{N_{0}} \sum_{k=1}^{2 N-r} \bar{\omega}_{k}^{2}(f)+\sum_{k=2 N-r+1}^{2 N} \frac{\bar{\omega}_{k}^{2}(f)}{\tilde{\lambda}_{k}(f)} .
\end{aligned}
$$

Again, using (C.5), the cross terms in (C.3) can be expressed as:

$$
\begin{aligned}
\tilde{\mathbf{h}}^{\dagger}(-f) \mathbf{R}_{\tilde{\mathbf{n}} \tilde{\mathbf{n}}}^{-\mathbf{1}}(f) \mathbf{P}^{\dagger} \tilde{\mathbf{h}}(f)= & \bar{\omega}^{\dagger}(-f) \tilde{\Delta}(f) \tilde{\omega}(f) \\
= & \frac{2}{N_{0}} \sum_{k=1}^{2 N-r} \bar{\omega}_{k}^{\dagger}(-f) \tilde{\omega}_{k}(f)+ \\
& \sum_{k=2 N-r+1}^{2 N} \frac{\bar{\omega}_{k}^{\dagger}(-f) \tilde{\omega}_{k}(f)}{\tilde{\lambda}_{k}(f)} .
\end{aligned}
$$


Substituting (C.6), (C.7), (C.8) in C.4, we get:

$$
\begin{aligned}
&\left|\tilde{\mathbf{H}}^{\dagger}(-f) \mathbf{R}_{\tilde{\mathbf{n}} \tilde{\mathbf{n}}}^{-\mathbf{1}}(f) \tilde{\mathbf{H}}(f)\right|= {\left[\frac{2}{N_{0}} \sum_{k=1}^{2 N-r} \tilde{\omega}_{k}^{2}(f)+\sum_{k=2 N-r+1}^{2 N} \frac{\tilde{\omega}_{k}^{2}(f)}{\tilde{\lambda}_{k}(f)}\right] \times } \\
& {\left[\frac{2}{N_{0}} \sum_{k=1}^{2 N-r} \bar{\omega}_{k}^{2}(f)+\sum_{k=2 N-r+1}^{2 N} \frac{\bar{\omega}_{k}^{2}(f)}{\tilde{\lambda}_{k}(f)}\right]-} \\
& \mid \frac{2}{N_{0}} \sum_{k=1}^{2 N-r} \bar{\omega}_{k}^{\dagger}(-f) \tilde{\omega}_{k}(f)+ \\
&\left.\sum_{k=2 N-r+1}^{2 N} \frac{\bar{\omega}_{k}^{\dagger}(-f) \tilde{\omega}_{k}(f)}{\tilde{\lambda}_{k}(f)}\right|^{2} \\
&=\frac{A(f)}{N_{0}}+\frac{B(f)}{N_{0}^{2}}+C(f)
\end{aligned}
$$

where $A(f), B(f), C(f)$ are obtained by expanding the squares in (C.9). Let us consider a mixed interference scenario where interference consists of a sum of $M_{1}$ PAM signals and $M_{2}$ QAM signals. Using (2.25), the interference autocorrelation can be expressed as:

$$
\mathbf{R}_{\tilde{\mathbf{i i}}}(f)=\underbrace{\sum_{l=1}^{M_{1}} \tilde{\mathbf{g}}^{l}(f) \tilde{\mathbf{g}}^{l \dagger}(-f)}_{\text {PAM CCI contribution }}+\underbrace{\sum_{m=1}^{M_{2}} \tilde{\mathbf{G}}^{m}(f) \tilde{\mathbf{G}}^{m \dagger}(-f)}_{\text {QAM CCI contribution }} .
$$

Since we are primarily interested in the rank of the $\mathbf{R}_{\widetilde{\mathbf{i i}}}(f)$ we can treat each QAM interferer as equivalent to two PAM interferers to determine the rank of ICM. With this equivalent model, the effective number of PAM interferers becomes $M_{1}+2 M_{2}$. In this case, using straightforward algebra, the rank of $\mathbf{R}_{\widetilde{\mathbf{i i}}}(f)$ can be expressed as: $r=\min \left(2 N, M_{1}+2 M_{2}\right)$. If $M_{1}+2 M_{2}<2 N$, the determinant expression (C.10) contains sum of terms containing $N_{0}, N_{0}^{2}$ in the denominator and a constant term. Note that in certain special cases the terms containing $B(f)$ may vanish (for example when $N=1, M_{1}=1$ ). Let us assume that $A(f), B(f)$ (if exists), $C(f)$ take finite 
values. Using the fact that $\tilde{\mathbf{H}}^{\dagger}(-f) \mathbf{R}_{\tilde{\mathbf{n}} \tilde{\mathbf{n}}}^{-1}(f) \tilde{\mathbf{H}}(f)$ is positive definite, as $N_{0} \rightarrow 0$, we see that

$$
\left|\tilde{\mathbf{H}}^{\dagger}(-f) \mathbf{R}_{\tilde{\mathbf{n}} \tilde{\mathbf{n}}}^{-1}(f) \tilde{\mathbf{H}}(f)\right| \rightarrow \infty
$$

Therefore, we say that, the receiver provides complete interference suppression when $M_{2}+2 M_{2}<2 N$. Note that in the opposite case when $M_{1}+2 M_{2} \geq 2 N$, the ICM has full rank i.e. $r=2 N$. In that case the terms containing $A(f), B(f)$ vanish. Consequently, the GSNR will be limited by the third term in $C(f)$ which is limited by interference power rather than thermal noise. In this case the system offers a partial IC benefit only.

When $\mathbf{R}_{\widetilde{\mathbf{i}}}(f)$ is rank deficient, the system cannot ensure infinite SNR gain in the special case when: $A(f)=B(f)=0$. When the CSI of signal and interference are random (mutually independent) and when the real and imaginary parts of the channel are statistically independent (as in Rayleigh fading channels), the above mentioned singular conditions (i.e. $A(f)=B(f)=0$ ) occur with small probability. In that case, one can say that, WL receivers provide full IC with probability nearly 1 in random channels. 


\section{APPENDIX D}

\section{MSE EQUIVALENCE}


D.1 MSE Equivalence between Conventional and WL receivers for QAM Systems

In this section we compare the MSE for WL and conventional methods when the noise is AWGN. We consider the analysis for DFE case only. The results can be extended to LE. Let

$$
\mathbf{P}(f)=\tilde{\mathbf{H}}^{\dagger}(-f) \tilde{\mathbf{H}}(f)+s N_{0} \mathbf{I} .
$$

Using (2.10) and letting $D=e^{-j 2 \pi f T}$, we get:

$$
\mathbf{P}(f)=\sum_{l=1}^{s} \sum_{m=1}^{N} \mathbf{A}
$$

where

$$
\mathbf{A}=\left[\begin{array}{cc}
\left|h_{I, l}^{m}(f)\right|^{2}+\left|h_{Q, l}^{m}(f)\right|^{2}+N_{0} & h_{I, l}^{m \dagger}(-f) h_{Q, l}^{m}(f)-h_{Q, l}^{m \dagger}(f) h_{I, l}^{m}(f) \\
h_{Q, l}^{m \dagger}(-f) h_{I, l}(f)-h_{I, l}^{m \dagger}(f) h_{Q, l}(f) & \left|h_{I, l}(f)\right|^{2}+\left|h_{Q, l}(f)\right|^{2}+N_{0}
\end{array}\right] .
$$

The off-diagonal elements can be expressed as:

$$
\sum_{l=1}^{s} \sum_{m=1}^{N} h_{I, l}^{m \dagger}(-f) h_{Q, l}^{m}(f)-h_{Q, l}^{m \dagger}(f) h_{I, l}^{m}(f)=2 j \sum_{l=1}^{s} \sum_{m=1}^{N} \operatorname{Im}\left[h_{I, l}^{m \dagger}(-f) h_{Q, l}^{m}(f)\right] .
$$

Using (3.14), (3.15) the determinant of $\mathbf{P}(f)$ can be expressed as:

$$
|\mathbf{P}(f)|=a^{2}(f)-\bar{a}^{2}(f) .
$$

We can verify that the following holds:

- $a(f)$ is an even function

- $|P(f)|$ is an even function

- $\bar{a}(f)$ is a real valued odd function i.e. $\int_{-\frac{1}{2 T}}^{\frac{1}{2 T}} \bar{a}(f) d f=0$

For a conventional signal model, we can define

$$
Q(f) \triangleq \mathbf{h}^{\dagger}(-f) \mathbf{h}(f)+s N_{0} .
$$


Using (2.8), we can express $Q(f)$ as

$$
\begin{aligned}
Q(f) & =\mathbf{h}(f)^{\dagger} \mathbf{h}(f)+s N_{0} \\
& =\sum_{l=1}^{s} \sum_{m=1}^{N}\left|h_{I, l}^{m}(f)\right|^{2}+\left|h_{Q, l}^{m}(f)\right|^{2}-2 \sum_{l=1}^{s} \sum_{m=1}^{N} \operatorname{Im}\left[h_{I, l}^{m \dagger}(-f) h_{Q, l}^{m}(f)\right]+N_{0} \\
& =a(f)-\bar{a}(f) .
\end{aligned}
$$

For a non-zero constant noise variance $N_{0}$ we have

$$
Q(f)=\mathbf{h}^{\dagger}(-f) \mathbf{h}(f)+s N_{0}>0
$$

By expressing expressing $Q(f)$ in terms of $a(f), \bar{a}(f)$ (D.2) we get

$$
Q(f)=a(f)-\bar{a}(f)>0 \Rightarrow \frac{\bar{a}(f)}{a(f)} \Rightarrow \frac{\bar{a}^{2}(f)}{a^{2}(f)}<1
$$

Using (A.1), we can express the MSE for conventional DFE as

$$
\mathrm{MSE}_{\text {Conv DFE }}=N_{0} e^{T \int_{-\frac{1}{2 T}}^{\frac{1}{2 T}} \ln \frac{1}{a(f)} d f+T \int_{-\frac{1}{2 T}}^{\frac{1}{2 T}}\left[\frac{\bar{a}^{2}(f)}{2 a^{2}(f)}+\frac{\bar{a}^{4}(f)}{4 a^{4}(f)}+. .\right] d f} .
$$

Next, recall that the geometric MSE for the WL DFE is given by (4.7):

$$
\begin{aligned}
\text { GMSE }_{\text {WL DFE QAM }} & =e^{-\frac{T}{2} \int_{-\frac{1}{2 T}}^{\frac{1}{2 T}} \ln \left|\mathbf{R}_{\tilde{\mathbf{x}} \tilde{\mathbf{x}}}^{-1}(f)+\tilde{\mathbf{H}}^{\dagger}(-f) \mathbf{R}_{\tilde{\mathbf{n}} \tilde{\mathbf{n}}}^{-1}(f) \tilde{\mathbf{H}}(f)\right| d f} \\
& =e^{-\frac{T}{2} \int_{-\frac{1}{2 T}}^{\frac{1}{2 T}} \ln \left|\frac{2 \mathbf{P}(f)}{N_{0}}\right| d f} \\
& =e^{-\frac{T}{2} \int_{-\frac{1}{2 T}}^{\frac{1}{2 T}} \ln \left[\frac{4\left(a^{2}(f)-\bar{a}^{2}(f)\right)}{N_{0}^{2}}\right] d f} \\
& =e^{\ln \frac{N_{0}}{2}+T \int_{-\frac{1}{2 T}}^{\frac{1}{2 T}} \ln \frac{1}{a(f)} d f-\frac{T}{2} \int_{-\frac{1}{2 T}}^{\frac{1}{2 T}} \ln \left[1-\frac{\bar{a}^{2}(f)}{a^{2}(f)}\right] d f} \\
& =\frac{N_{0}}{2} e^{T \int_{-\frac{1}{2 T}}^{\frac{1}{2 T}} \ln \frac{1}{a(f)} d f+T \int_{-\frac{1}{2 T}}^{\frac{1}{2 T}}\left[\frac{\bar{a}^{2}(f)}{2 a^{2}(f)}+\frac{\bar{a}^{4}(f)}{4 a^{4}(f)}+. .\right] d f} \\
& =\frac{\mathrm{MSE}_{\mathrm{Conv} \mathrm{DFE}}}{2} .
\end{aligned}
$$

In (D.7), we invoked an infinite series of type $-\ln (1-x)=x+\frac{x^{2}}{2}+\frac{x^{3}}{3}+.$. for $|x|<1$. Since the GMSE is defined as per branch MSE, since the noise is white (the error correlation matrix at WL DFE output must be a diagonal matrix with 
equal entries) we can define the total MSE as MSE $_{\text {WL DFE QAM }}=2$ GMSE $_{\text {Conv DFE QAM }}$ which is equal to the MSE produced by the conventional receiver. We would like to remark here that the MSE equivalence between conventional and WL methods can be shown rather easily if we formulate the WL QAM equalization problem using the complex and complex-conjugate quantities. 


\section{APPENDIX E}

\section{WISHART MATRICES}




\section{E.1 Wishart and Anti-Wishart Matrices}

In the following we use the definitions and proofs from [59] regarding Wishart matrices and apply these results to determine the statistical properties of ICM.

Definition A random matrix of form $\mathbf{A}=\mathbf{Z}^{\dagger} \mathbf{Z}=\sum_{k=1}^{n} Z_{k}^{\dagger} Z_{k}$ is called a real Wishart matrix denoted as: $\mathbf{W}_{m}\left(n, \frac{1}{\lambda} \mathbf{I}\right)$ if the elements of $\mathbf{Z}$ is of form: $\mathbf{Z}=\left[\begin{array}{c}Z_{1} \\ : \\ Z_{n}\end{array}\right]$ where each individual vector $Z_{i}=\left[z_{i, 1}, . ., z_{i, m}\right]$ is a real i.i.d. Gaussian random vector: $N\left(0, \frac{1}{\beta} \mathbf{I}\right)$ and $n \geq m$.

Theorem E.1.1 The probability distribution of eigenvalues $\tilde{\lambda}_{1}, . ., \tilde{\lambda}_{m},\left(\tilde{\lambda}_{1}>\tilde{\lambda}_{2} . \tilde{\lambda}_{m}>\right.$ 0) of $\mathbf{A}=\mathbf{W}_{m}\left(n, \frac{1}{\beta} \mathbf{I}\right),(n \geq m)$ is

$$
\frac{\pi^{\frac{m^{2}}{2}}}{(2 \beta)^{\frac{m n}{2}} \Gamma_{m}\left(\frac{m}{2}\right) \Gamma_{m}\left(\frac{n}{2}\right)} e^{\frac{-1}{2 \lambda} \sum_{i=1}^{m} \tilde{\lambda}_{i}} \prod_{i=1}^{m} \tilde{\lambda}_{i}^{\frac{(n-m-1)}{2}} \prod_{i<j}^{m}\left(\tilde{\lambda}_{i}-\tilde{\lambda}_{j}\right)
$$

where $\Gamma_{m}($.$) denotes the multivariate gamma function defined as$

$$
\Gamma_{m}(a)=(\pi)^{\frac{m(m-1)}{4}} \prod_{i=1}^{m} \Gamma\left[a-\frac{1}{2}(i-1)\right], \quad \Gamma(x)=\int_{0}^{\infty} t^{x-1} e^{-t} d t x>0
$$

is the gamma function.

Proof See [59].

Theorem E.1.2 The expected value of products of the square roots of the eigenvalues of a Wishart matrix is: $E\left[\prod_{i=1}^{m} \sqrt{\tilde{\lambda}_{i}}\right]=(\beta)^{\frac{m}{2}} \prod_{i=1}^{m} \frac{\Gamma\left(\frac{n-i+1}{2}+\frac{1}{2}\right)}{\Gamma\left(\frac{n-i+1}{2}\right)}$.

Proof Theorem 3.2.15 of [59] considered $E[|\mathbf{A}|]$. A straight forward modification of that results gives the stated result.

Definition The singular matrix $\overline{\mathbf{A}}=\mathbf{Z} \mathbf{Z}^{\dagger}$ for $n>m$ is called an anti-Wishart matrix. Theorem E.1.3 The first $m$ eigenvalue of anti-Wishart matrix $\overline{\mathbf{A}}$ are equal to $\tilde{\lambda}_{1}, . ., \tilde{\lambda}_{m}$ and the the remaining $n-m$ eigenvalues are zero. These non-zero eigenvalues are same as the eigenvalues of the Wishart matrix $\mathbf{A}=\mathbf{Z}^{\dagger} \mathbf{Z}: \mathbf{W}_{n}\left(m, \frac{1}{\beta} \mathbf{I}\right)$. 
Proof The proof is trivial.

Theorem E.1.4 The expected value of the product of non-zero eigenvalues (pseudodeterminant) of $\mathbf{R}_{\tilde{i} i}$ is: $E\left[\prod_{l=1}^{m} \sqrt{\tilde{\lambda}_{l}}\right]=\left(\frac{I_{0}}{2}\right)^{\frac{m}{2}} \prod_{i=1}^{m} \frac{\Gamma\left(\frac{n-i+1}{2}+\frac{1}{2}\right)}{\Gamma\left(\frac{n-i+1}{2}\right)} m=\min (2 N, M)$, $n=\max (2 N, M)$.

Proof In this case

$$
\mathbf{R}_{\tilde{i}}=\sum_{l=1}^{M} \tilde{\mathbf{g}}^{l} \tilde{\mathbf{g}}^{l \dagger}
$$

Recall that the channel vector of $j$ th interferer $\tilde{\mathbf{g}}^{j}$ is a column vector of length $2 N$ whose elements are assumed to be i.i.d. zero mean real Gaussian random variables of variance $\frac{I_{0}}{2}$ per element. The interference correlation matrix (ICM) $\mathbf{R}_{\tilde{i} \tilde{i}}=\sum_{j=1}^{M} \tilde{\mathbf{g}}^{j} \tilde{\mathbf{g}}^{j \dagger}$ has full rank when the number of interferers $M$ is greater than or equal to $2 N$. In this case $\mathbf{R}_{\tilde{i} \tilde{i}}$ becomes a real Wishart matrix denoted as: $\mathbf{W}_{2 N}\left(M, \frac{I_{0}}{2} \mathbf{I}\right)$. When the number of interferers $M$ is less then $2 N$, the ICM is rank deficient. In this case $\mathbf{R}_{\tilde{i} i}$ becomes ani-Wishart. Combining the Wishart and anti-Wishart cases, and applying Theorems E.1.2, Theorem E.1.4, we can express the expected value of the product of square root of non-zero eigenvalues $\tilde{\lambda}_{l}$ of $\mathbf{R}_{\tilde{i} \tilde{i}}$ as

$$
E\left[\prod_{l=1}^{m} \sqrt{\tilde{\lambda}_{l}}\right]=\left(\frac{I_{0}}{2}\right)^{\frac{m}{2}} \prod_{i=1}^{m} \frac{\Gamma\left(\frac{n-i+1}{2}+\frac{1}{2}\right)}{\Gamma\left(\frac{n-i+1}{2}\right)} \quad m=\min (2 N, M), \quad n=\max (2 N, M)(\mathrm{E} .3)
$$

where $m$ denotes the rank of $\mathbf{R}_{\tilde{i}}$. 


\section{REFERENCES}

[1] G.J.Foschini and M.J.Gans, "On limits of wireless communication in a fading environment when using multiple antennas," in Wireless personal communications, Mar. 1998, pp. 311-335.

[2] E.Telatar, "Capacity of multi-antenna gaussian channels," in ATT-Bell Labs Internal Tech. Memo, 1995, pp. 585-595.

[3] V.Tarokh, N. Seshadri, and A.R.Calderbank, "Space-Time codes for high data rate wireless communication:performance criterion and code construction," IEEE Trans. Inform. Theory, vol. 44, pp. 744-65, Mar. 1998.

[4] S.L.Ariyavisitakul, J.H.Winters, and I.Lee, "Optimum space-time processors with dispersive interference: Unified analysis and required filter span," IEEE Trans. Commun., vol. 47, pp. 1073-83, 1999.

[5] J. Chioffi, EE:379 Stanford Class Notes. [Online]. Available: http://www.stanford.edu/class/ee379a/

[6] G.D.Forney, "Maximum-likelihood sequence estimation of digital sequences in the presence of intersymbol interference," IEEE Trans. Inform. Theory, vol. 18, pp. 363-78, May 1972.

[7] G. Ungerboeck, "Adaptive maximum-likelihood receiver for carrier-modulated data-transmission systems," IEEE Trans. Commun., vol. 22, pp. 624-36, 1974.

[8] L.R.Bahl, J. Cocke, F. Jelinek, and J. Raviv, "Optimal decoding of linear codes for minimizing symbol error rate," IEEE Trans. Inform. Theory, pp. 284-87, 1974. 
[9] M.Y.Eyuboglu and S. Quereshi, "Reduced state sequence estimation with setpartitioning and decision feedback," IEEE Trans. Commun., vol. 36, pp. 13-20, Jan. 1988.

[10] W. H. Gerstacker and R. Schober, "Equalization concepts for EDGE," IEEE Trans. Wireless Commun., vol. 1, pp. 190-99, Jan. 2002.

[11] W. V. Etten, "Maximum likelihood receiver for multiple-channel transmission systems," IEEE Trans. Commun., vol. 24, pp. 276-83, Feb. 1976.

[12] K. Giridhar, "Nonlinear techniques for joint estimation of cochannel signals," IEEE Trans. Commun., vol. 45, pp. 473-84, Dec. 1995.

[13] S. Verdu, "Minimum probability of error for asynchronous gaussain multipeaccess channels," IEEE Trans. Inform. Theory, vol. 32, pp. 85-96, Jan. 1986.

[14] A. Duel-Hallen, "Equalizers for multiple input/multiple output channels and PAM systems with cyclostationary input sequences," IEEE J. Select. Areas Commun., vol. 10, pp. 630-39, 1992.

[15] S.J.Grant and J.K.Cavers, "Performance enhancement through joint detection of cochannel signals using diversity arrays," IEEE Trans. Commun., vol. 46, pp. 1038-49, Aug. 1998.

[16] Y. Li, J.H.Winters, and N.R.Sollenberger, "Spatio temporal equalization for IS136 TDMA systems with rapid dispersive fading and cochannel interference," IEEE Trans. Veh. Technol., vol. 48, pp. 1182-94, 1999.

[17] J.H.Winters, R.D.Gitlin, and J. Salz, "The impact of antenna diversity on the capacity of wireless communication systems," IEEE Trans. Commun., vol. 42, pp. 1740-51, 1994.

[18] B. Picinbono and P. Chevalier, "Widely linear estimation with complex data," IEEE Trans. Signal Processing, vol. 43, pp. 2030-2033, Aug. 1995. 
[19] F. Neeser and J. Massey, "Proper complex random processes with application to information theory," IEEE Trans. Inform. Theory, vol. 39, pp. 1293-1302, 1993.

[20] W.M.Brown and R.B.Crawe, "Conjugate linear filtering," IEEE Trans. Inform. Theory, vol. 15, pp. 462-465, 1969.

[21] W.A.Gardner, Cyclostationarity in Communications and Signal Processing. IEEE Press, 1994.

[22] P. Laurent, "Exact and approximate construction of digital phase modualtions by superposition of amplitude modulated pulses," IEEE Trans. Commun., vol. 34, pp. 150-160, 1986.

[23] Y.C.Yoon and H. Leib, "Maximizing SNR in improper complex noise and application to CDMA," IEEE Commun. Lett., vol. 1, pp. 5-8, Jan. 1997.

[24] A. Lampe and M. Breiling, "Asymptotic analysis of widely linear MMSE multiuser detection-complex vs real modulation," in Information Theory Workshop, 2001, pp. 55-57.

[25] A. Lampe, R. Schober, W. H. Gerstacker, and J. Huber, "A novel iterative multiuser detector for complex modulation schemes," IEEE J. Select. Areas Commun., vol. 20, pp. 339-50, Feb. 2002.

[26] G. Gelli, L. Paura, and A. Ragozini, "Widely linear multiuser detection," IEEE Commun. Lett., vol. 4, pp. 187-9, June 2000.

[27] D. Darsena, G.Gelli, L. Paura, and F.Verde, "Widely linear equalization and blind channel identification for interference-contaminated multicarrer systems," IEEE Trans. Signal Processing, vol. 53, pp. 1163-77, 2005.

[28] W. H. Gerstacker, R. Schober, and A. Lampe, "Receivers with widely linear processing for frequency-selective channels," IEEE Trans. Commun., vol. 51, pp. 1512-22, 2003. 
[29] W. H. Gerstacker, F. Obernosterer, R. Schober, A. Lehmann, A. Lampe, and P.Gunerben, "Equalization concepts for alamoutis space-time block code," IEEE Trans. Commun., vol. 52, pp. 1178-90, 2004.

[30] P. Chevalier and F.Pipon, "New insights into optimal widely linear array receivers for demodulation of BPSK,MSK, and GMSK corrupted by noncircular interferers-application to SAIC," IEEE Trans. Signal Processing, vol. 54, pp. 870-883, Mar. 2006.

[31] H. Trigui and D. Slock, "Cochannel interference cancellation within the current GSM standard," in Proc. Universal Personal Communications, Oct. 1998, pp. $511-15$.

[32] W.A.Gardner and C.W.Reid, "Making the most out of spectral redundancy in GSM: Cheap CCI suppression," in Proc. Asilomar on Signals, Systems and Computers, Oct. 2001, pp. 883-889.

[33] P.Ranta, A. Hottinen, and Z.C.Honkasalo, "Co-channel interference cancelling receiver for TDMA mobile systems," in $I C C, 1995$, pp. 17-21.

[34] J. Zhang and B. V. V. A.M. Sayeed, "Reduced state MIMO sequence detection with application to EDGE systems," IEEE Trans. Wireless Commun., vol. 4, pp. 1040-49, May 2005.

[35] T.Guess and M.K.Varanasi, "An information-theoretic framework for deriving canonical decision-feedback receivers in gaussian channels," IEEE Trans. Inform. Theory, vol. 51, pp. 173-187, Jan. 2005.

[36] J. Cioffi, G.Dudevoir, M.V.Eyuboglu, and G.D.Forney, "MMSE decisionfeedback equalizers and coding-part 1: Equalization results," IEEE Trans. Commun., vol. 43, pp. 2582-94, 1995.

[37] — _ "MMSE decision-feedback equalizers and coding-part 2: Coding results," IEEE Trans. Commun., vol. 43, pp. 2595-2603, 1995. 
[38] P. Schreier, L. Scharf, and C.T.Mullis, "Detection and estimation of improper complex random signals," IEEE Trans. Inform. Theory, vol. 51, pp. 306-312, Jan. 2005.

[39] N. Al-Dhahir, "MMSE decision-feedback equalizers: Finite length results," IEEE Trans. Commun., vol. 41, pp. 961-75, 1995.

[40] T. Kailath, A. Sayed, and B. Hassibi, Linear Estimation. NJ: Prentice Hall, 2000.

[41] J.M.Cioffi, P.H.Algoet, and P.S.Chow, "Combined equalization and coding with finite-length decision feedback equalization," in GLOBECOM, Dec. 1990, pp. $1664-1668$.

[42] K.Kuchi and V.K.Prabhu, "Use of conventional and widely linear equalizers for PAM-type signals with ISI," IEEE Trans. Commun., submitted for publication.

[43] N. Wiener and P. Masani, "The predeiction theory of multivariate stochastic processes-1," Acta Math., vol. 98, pp. 112-50, Nov. 1957.

[44] J. Yang and S. Roy, "Joint transmitter and receiver optimization for multipleinput-multiple-output systems with decision feedback," IEEE Trans. Inform. Theory, vol. 40, pp. 1334-47, 1994.

[45] N. Al-Dhahir and A. Sayed, "The finite length multi-input multi-output MMSEDFE," IEEE Trans. Signal Processing, vol. 48, pp. 2921-36, Oct. 2000.

[46] J.H.Winters, "Optimum combing in digital mobile radio with cochannel interference," IEEE J. Select. Areas Commun., pp. 528-39, 1984.

[47] M. Chiani, M.Z.Win, A. Zanella, R.K.Malik, and J.H.Winters, "Bounds and approximation for optimum combining of signals in the presence of multiple cochannel interferers," IEEE Trans. Commun., vol. 51, pp. 296-307, Feb. 2003. 
[48] A.Shah, A.H.Haimovich, M.K.Simon, and M.S.Alouini, "Exact bit error probability for optimum combing with a rayleigh fadig gaussain cochannel interferer," IEEE Trans. Commun., vol. 48, pp. 908-912, 2000.

[49] A.Shah and A.H.Haimovich, "Performance analysis of optimum combing in wireless communication with rayleigh fading and cochannel interference," IEEE Trans. Commun., vol. 46, pp. 473-479, 1998.

[50] E.Villier, "Multipath performance of adaptive antennas with multiple interferers and correlated rayleigh fadings," IEEE Trans. Veh. Technol., vol. 48, pp. 342$352,1999$.

[51] _ _ "Performance analysis of optimum combing with multiple interferers in flat rayleigh fading," IEEE Trans. Commun., vol. 46, pp. 1503-1510, 1999.

[52] R.A.Horn and C.R.Johnson, Matrix Analysis. Cambridge University Press, 1990.

[53] J.G.Proakis, Digital Communications. Mc Graw Hill, 2000.

[54] M. Chiani, D.Dardari, and M.K.Simon, "New exponential bounds and approximations for the computation of error probability in fading channels," IEEE Trans. Wireless Commun., vol. 2, pp. 840-45, 2003.

[55] C.Douillard, A. Picart, P.Didier, M. Jezequel, C. Berrou, and A. Glavieux, "Iterative correction of intersymbol interference: Turbo equalization," Oct. 1995, pp. $507-11$.

[56] M. Tuchler, A. Singer, and R. Koetter, "Minimum mean squared error equalization using a priori information," IEEE Trans. Signal Processing, vol. 50, pp. 673-83, Mar. 2002.

[57] Physical Layer Aspects for Evolved UTRA (Release 7), 3GPP Std. TR 25.814, 2005. 
[58] A.H.Sayed and T.Kailath, A Survey of Spectral Factorization Methods. [Online]. Available: http://www.ee.ucla.edu/asl/publications/journalarticles/nlaa2001.pdf/

[59] R. Murihead, Aspects of Multivariate Statistical Theory. Wiley Series in Probability and Mathematical Statistics, 1982. 


\section{BIOGRAPHICAL STATEMENT}

Kiran Kumar Kuchi was born in Kavali, India in 1974. He received B.Tech degree in electronics and communications engineering from S.V.University, Tirupati, India, in 1995, MS and PhD degrees in electrical engineering from the The University of Texas at Arlington, Arlington, Texas, USA, in 1997 and 2006 respectively. From 1997-2000, he was a senior research engineer at Motorola Labs, Fort Worth, Texas where he worked on the design and analysis of transmit antenna diversity systems for CDMA2000 standard. He has been working for Nokia, Irving, Texas since January 2000. At Nokia, he played an instrumental role in the development receiver algorithms for GSM/EDGE systems. Presently he is involved in research and development activities

related to $3.9 \mathrm{G}$ systems. His main research interests include modulation and coding techniques, wireless systems, and signal processing algorithms for multiple antenna systems. 